Supporting Information

\title{
Theoretical Investigation of the Stereoselective Stepwise Cope Rearrangement of a 3-Vinylmethylenecyclobutane
}

\author{
Yilei Zhao, Christopher P. Suhrada, Michael E. Jung, K. N. Houk \\ Department of Chemistry and Biochemistry, University of California, Los Angeles, CA 90095-1569
}

\section{Full citations}

\section{Reference 11}

Gaussian 03, revision C.02. Frisch, M. J.; Trucks, G. W.; Schlegel, H. B.; Scuseria, G. E.; Robb, M. A.; Cheeseman, J. R.; Montgomery, J. A., Jr.; Vevren, T.; Kudin, K. N.; Burant, J. C.; Millam, J. M.; Iyengar, S. S.; Tomasi, J.; Barone, V.; Mennucci, B.; Cossi, M.; Scalmani, G.; Rega, N.; Petersson, G. A.; Nakatsuji, H.; Hada, M.; Ehara, M.; Toyota, K.; Fukuda, R.; Hasegawa, J.; Ishida, M.; Nakajima, T.; Honda, Y.; Kitao, O.; Nakai, H.; Klene, M.; Li, X.; Knox, J. E.; Hratchian, H. P.; Cross, J. B.; Adamo, C.; Jaramillo, J.; Gomperts, R.; Stratmann, R. E.; Yazyev, O.; Austin, A. J.; Cammi, R.; Pomelli, C.; Ochterski, J. W.; Ayala, P. Y.; Morokuma, K.; Voth, G. A.; Salvador, P.; Dannenberg, J. J.; Zakrzewski, V. G.; Dapprich, S.; Daniels, A. D.; Strain, M. C.; Farkas, O.; Malick, D. K.; Rabuck, A. D.; Raghavachari, K.; Foresman, J. B.; Ortiz, J. V.; Cui, Q.; Baboul, A. G.; Clifford, S.; Cioslowski, J.; Stefanov, B. B.; Liu, G.; Liashenko, A.; Piskorz, P.; Komaromi, I.; Martin, R. L.; Fox, D. J.; Keith, T.; Al-Laham, M. A.; Peng, C. Y.; Nanayakkara, A.; Challacombe, M.; Gill, P. M. W.; Johnson, B.; Chen, W.; Wong, M. W.; Gonzalez, C.; Pople, J. A. Gaussian, Inc., Wallingford CT, 2004.

\section{Reference 12}

Gaussian 98, revision A.9. Frisch, M. J.; Trucks, G. W.; Schlegel, H. B.; Scuseria, G. E.; Robb, M. A.; Cheeseman, J. R.; Zakrzewski, V. G.; Montgomery, J. A., Jr.; Stratmann, R. E.; Burant, J. C.; Dapprich, S.; Millam, J. M.; Daniels, A. D.; Kudin, K. N.; Strain, M. C.; Farkas, O.; Tomasi, J.; Barone, V.; Cossi, M.; Cammi, R.; Mennucci, B.; Pomelli, C.; Adamo, C.; Clifford, S.; Ochterski, J.; Petersson, G. A.; Ayala, P. Y.; Cui, Q.; Morokuma, K.; Malick, D. K.; Rabuck, A. D.; Raghavachari, K.; Foresman, J. B.; Cioslowski, J.; Ortiz, J. V.; Baboul, A. G.; Stefanov, B. B.; Liu, G.; Liashenko, A.; Piskorz, P.; Komaromi, I.; Gomperts, R.; Martin, R. L.; Fox, D. J.; Keith, T.; Al-Laham, M. A.; Peng, C. Y.; Nanayakkara, A.; Challacombe, M.; Gill, P. M. W.; Johnson, B.; Chen, W.; Wong, M. W.; Andres, J. L.; Gonzalez, C.; Head-Gordon, M.; Replogle, E. S.; Pople, J. A. Gaussian, Inc., Pittsburgh PA, 1998.

\section{Reference 13}

Molcas, version 5.0. Andersson, K. ; Barysz, M.; Bernhardsson, A.; Blomberg, M. R. A.; Cooper, D. L.; Fleig, T.; Fülscher, M. P.; de Graaf, C.; Hess, B. A.; Karlström, G.; Lindh, R.; Malmqvist, P.-Å.; Neogrády, P.; Olsen, J.; Roos, B. O.; Sadlej, A. J.; Schimmelpfennig, B.; Schütz, M.; Seijo, L.; Serrano-Andrés, L.; Siegbahn, P. E. M.; Stålring, J.; Thorsteinsson, T.; Veryazov, V.; Widmark, P.-O. Lund University, Sweden, 2001. 


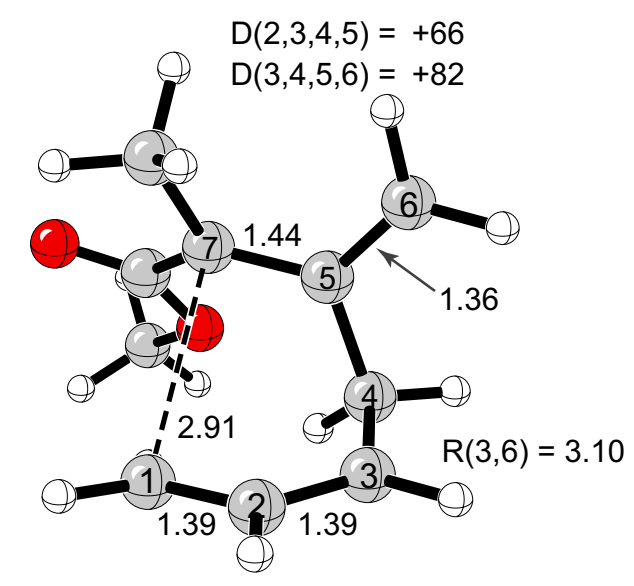

TS-Zd-2d-x-trans

(1.1)

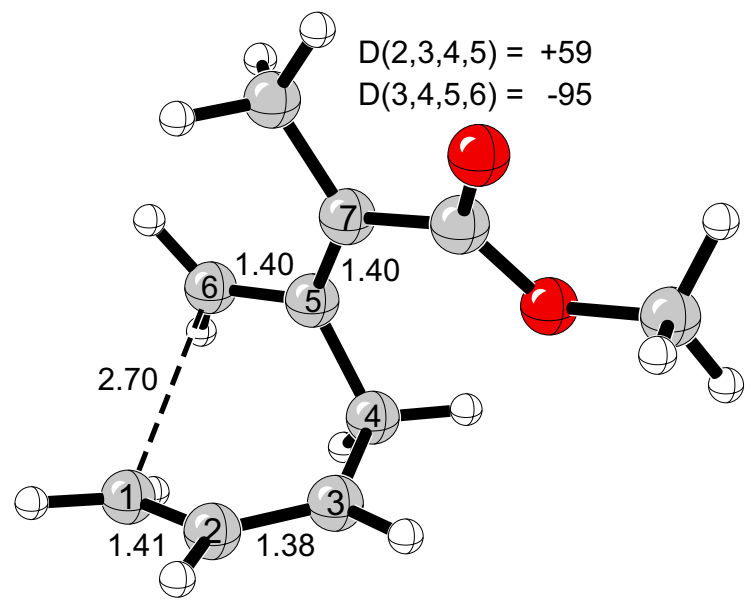

TS'-Zd-2d-x-trans

(4.1)

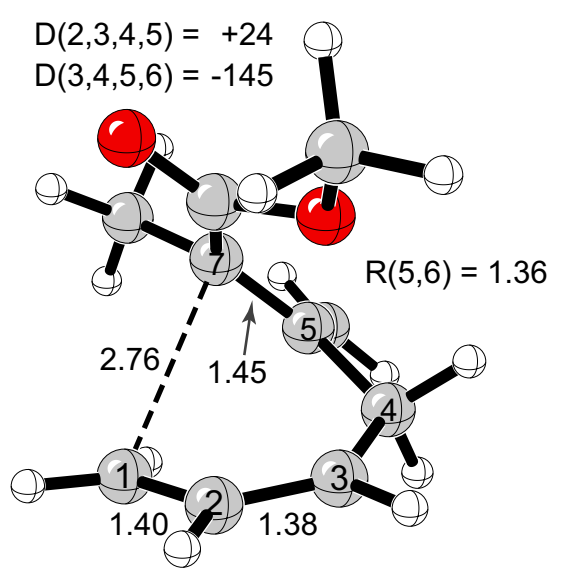

TS-Zd-2d-n-trans

(2.7)

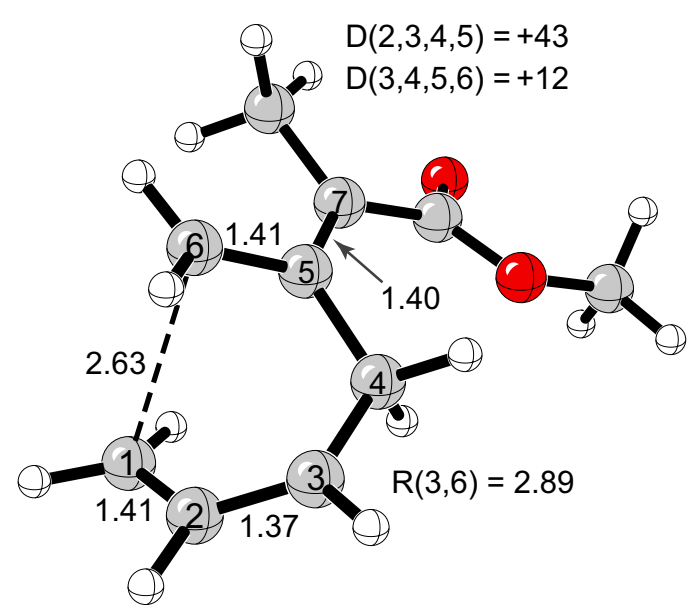

TS'-Zd-2d-n-trans

(4.0)

Supplement to Figure 9. $s$-trans-Carbonyl conformers of transition states pictured in Figure 9. UB3LYP/6-31+G(d) enthalpies listed in $\mathrm{kcal} / \mathrm{mol}$ relative to TS-Zd-2d-x-cis. 


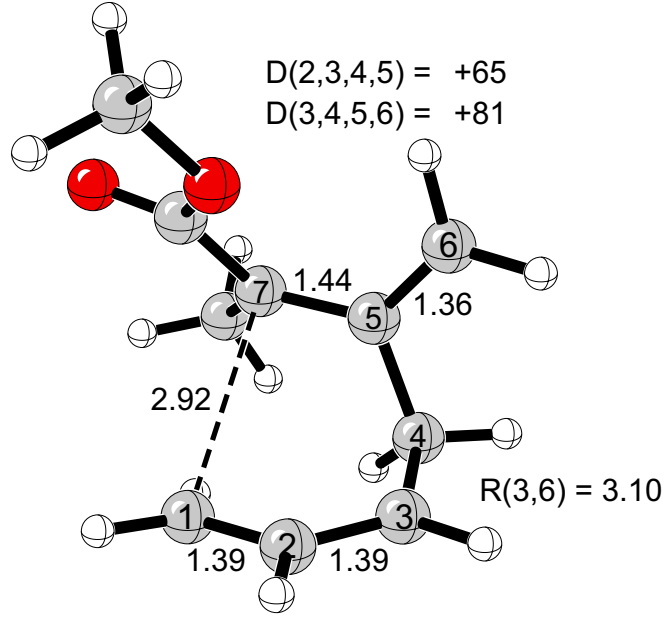

TS-Ed-2d-x-trans

(2.2)

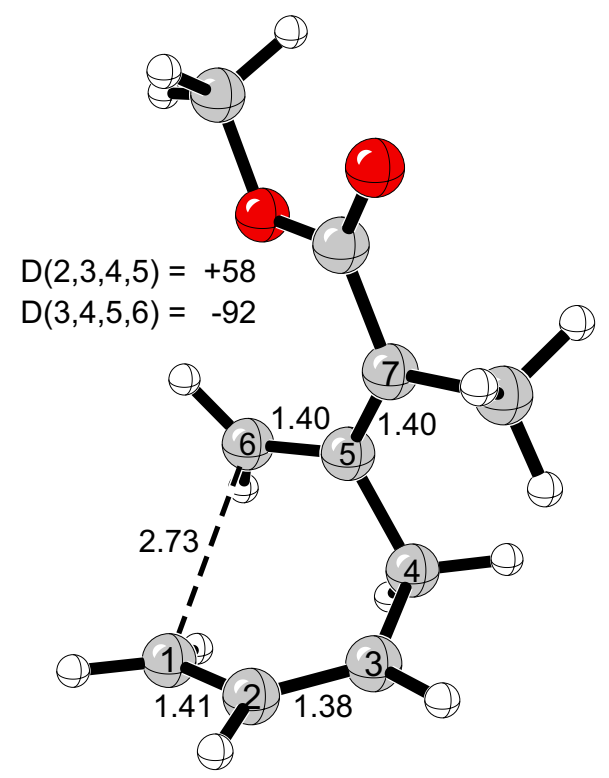

TS'-Ed-2d-x-trans

(4.8)

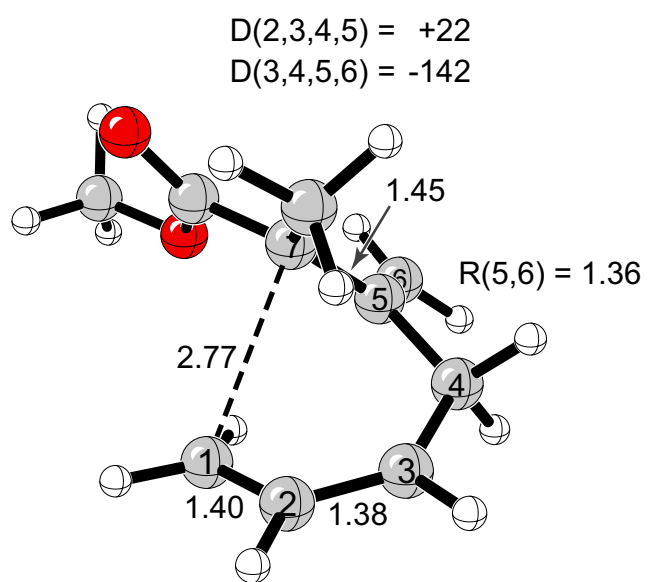

TS-Ed-2d-n-trans

(2.8)

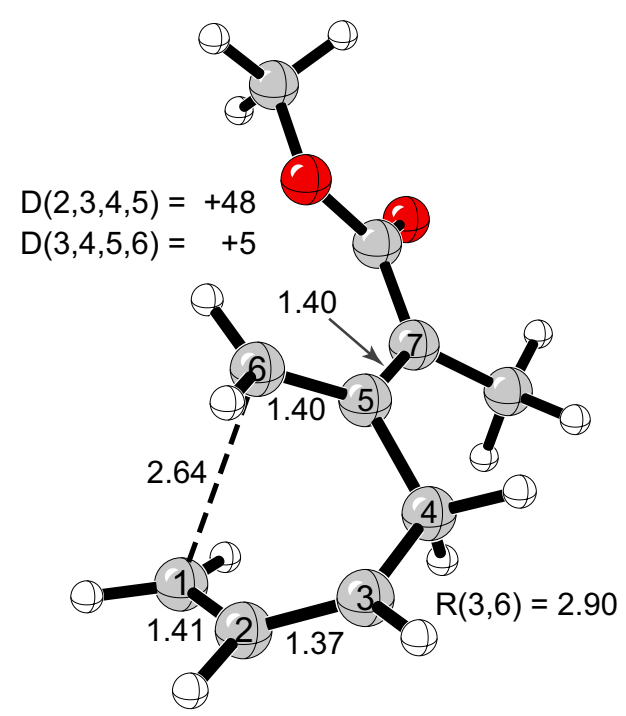

TS'-Ed-2d-n-trans

(4.2)

Supplement to Figure 10. s-trans-Carbonyl conformers of transition states pictured in Figure 10. UB3LYP/6-31+G(d) enthalpies listed in $\mathrm{kcal} / \mathrm{mol}$ relative to TS-Zd-2d-x-cis. 
RB3LYP / 6-31+G(d) :

$\begin{array}{lrrr}\text { C } & -2.71139 & -0.51734 & -0.36233 \\ \mathrm{C} & -1.92523 & 0.45270 & 0.11673 \\ \mathrm{C} & -0.49894 & 0.31109 & 0.55330 \\ \mathrm{C} & 0.59686 & 1.14280 & -0.20567 \\ \mathrm{C} & 1.49345 & -0.08374 & -0.12005 \\ \mathrm{C} & 2.81147 & -0.26748 & -0.17427 \\ \mathrm{C} & 0.31652 & -0.98134 & 0.23514 \\ \mathrm{H} & 0.95224 & 2.06732 & 0.26307 \\ \mathrm{H} & 0.28953 & 1.36361 & -1.23661 \\ \mathrm{H} & 0.45557 & -1.70771 & 1.04327 \\ \mathrm{H} & -0.07662 & -1.50637 & -0.64509 \\ \mathrm{H} & -2.33761 & 1.45963 & 0.21580 \\ \mathrm{H} & -2.36338 & -1.54156 & -0.47694 \\ \mathrm{H} & -0.42599 & 0.53716 & 1.62626 \\ \mathrm{H} & 3.25706 & -1.24654 & -0.01000 \\ \mathrm{H} & 3.49293 & 0.55455 & -0.38385 \\ \mathrm{H} & -3.74015 & -0.32026 & -0.65290\end{array}$

$E(R B+H F-L Y P)=-272.700154780$

$383 \mathrm{~K}, 1.00 \mathrm{~atm}$, freq's scaled by 1.0000

Zero-point correction $=0.148046$

Thermal correction to $\mathrm{U}=0.159495$

Thermal correction to $\mathrm{H}=0.160708$

Thermal correction to $\mathrm{G}=0.104826$

$\mathrm{E}(\operatorname{CASPT} 2(6,6) / 6-31 \mathrm{G}(\mathrm{d}))=-271.727058024$

$(6,6)$ CASSCF / 6-31+G* :

$\begin{array}{lrrr}\mathrm{C} & -2.72405 & -0.52361 & -0.35523 \\ \mathrm{C} & -1.92289 & 0.45893 & 0.08368 \\ \mathrm{C} & -0.50603 & 0.31036 & 0.55428 \\ \mathrm{C} & 0.62166 & 1.15677 & -0.18335 \\ \mathrm{C} & 1.49219 & -0.08269 & -0.10779 \\ \mathrm{C} & 2.80920 & -0.29005 & -0.19082 \\ \mathrm{C} & 0.31125 & -0.97005 & 0.23960 \\ \mathrm{H} & 0.97751 & 2.04964 & 0.31862 \\ \mathrm{H} & 0.33865 & 1.40689 & -1.20195 \\ \mathrm{H} & 0.44319 & -1.68046 & 1.04815 \\ \mathrm{H} & -0.06328 & -1.49887 & -0.63147 \\ \mathrm{H} & -2.31844 & 1.46269 & 0.13294 \\ \mathrm{H} & -2.39836 & -1.54694 & -0.41787 \\ \mathrm{H} & -0.45799 & 0.52546 & 1.61863 \\ \mathrm{H} & 3.23433 & -1.26864 & -0.04711 \\ \mathrm{H} & 3.49239 & 0.51437 & -0.40353 \\ \mathrm{H} & -3.73604 & -0.32218 & -0.65852\end{array}$

$\mathrm{E}(\operatorname{CASSCF}(6,6) / 6-31+\mathrm{G}(\mathrm{d}))=-270.8998209589$

$\mathrm{E}(\operatorname{CASPT} 2(6,6) / 6-31 \mathrm{G}(\mathrm{d}))=-271.7279160652$
RB3LYP / 6-31+G(d) :

$\begin{array}{lrrr}\mathrm{C} & -1.05266 & 0.01804 & 0.10136 \\ \mathrm{C} & -2.27607 & -0.07038 & -0.43434 \\ \mathrm{C} & 1.14044 & 1.24118 & -0.03684 \\ \mathrm{C} & -0.33374 & 1.33788 & 0.29084 \\ \mathrm{C} & 1.77268 & 0.08463 & -0.27426 \\ \mathrm{C} & 1.09028 & -1.26051 & -0.21301 \\ \mathrm{C} & -0.25460 & -1.18600 & 0.54197 \\ \mathrm{H} & -2.82278 & 0.81031 & -0.76555 \\ \mathrm{H} & -2.77682 & -1.02888 & -0.55536 \\ \mathrm{H} & -0.44481 & 1.67395 & 1.33673 \\ \mathrm{H} & -0.80506 & 2.11848 & -0.32134 \\ \mathrm{H} & 2.83283 & 0.09568 & -0.52602 \\ \mathrm{H} & 0.92363 & -1.63762 & -1.23399 \\ \mathrm{H} & 1.69332 & 2.17916 & -0.08013 \\ \mathrm{H} & -0.82867 & -2.10864 & 0.39898 \\ \mathrm{H} & -0.03923 & -1.09920 & 1.61824 \\ \mathrm{H} & 1.74960 & -1.99232 & 0.27416\end{array}$

$E(R B+H F-L Y P)=-272.741375392$

$383 \mathrm{~K}, 1.00 \mathrm{~atm}$, freq's scaled by 1.0000

Zero-point correction $=0.150837$

Thermal correction to $\mathrm{U}=0.161191$

Thermal correction to $\mathrm{H}=0.162404$

Thermal correction to $\mathrm{G}=0.109590$

$\mathrm{E}(\operatorname{CASPT} 2(6,6) / 6-31 \mathrm{G}(\mathrm{d}))=-271.771664858$

$(6,6) \mathrm{CASSCF} / 6-31+\mathrm{G}^{*}:$

$\begin{array}{lrrr}\mathrm{C} & -1.04244 & 0.01889 & 0.10399 \\ \mathrm{C} & -2.28208 & -0.07472 & -0.44385 \\ \mathrm{C} & 1.13857 & 1.24348 & -0.03780 \\ \mathrm{C} & -0.33292 & 1.34058 & 0.29628 \\ \mathrm{C} & 1.77210 & 0.08246 & -0.26916 \\ \mathrm{C} & 1.08497 & -1.26096 & -0.21285 \\ \mathrm{C} & -0.24587 & -1.18346 & 0.54785 \\ \mathrm{H} & -2.82122 & 0.79726 & -0.77183 \\ \mathrm{H} & -2.77182 & -1.02540 & -0.56485 \\ \mathrm{H} & -0.44096 & 1.66507 & 1.33183 \\ \mathrm{H} & -0.79818 & 2.11055 & -0.31216 \\ \mathrm{H} & 2.81949 & 0.09292 & -0.52236 \\ \mathrm{H} & 0.91045 & -1.62174 & -1.22505 \\ \mathrm{H} & 1.68925 & 2.16863 & -0.08342 \\ \mathrm{H} & -0.81534 & -2.09685 & 0.41089 \\ \mathrm{H} & -0.03401 & -1.09361 & 1.61241 \\ \mathrm{H} & 1.73911 & -1.98808 & 0.26154\end{array}$

$\mathrm{E}(\operatorname{CASSCF}(6,6) / 6-31+\mathrm{G}(\mathrm{d}))=-270.950992821$

$\mathrm{E}(\operatorname{CASPT} 2(6,6) / 6-31 \mathrm{G}(\mathrm{d}))=-271.7708574682$ 
TS-1-3

UB3LYP / 6-31+G (d) :

$\begin{array}{lrrr}\mathrm{C} & -2.75682 & -0.16943 & -0.47475 \\ \mathrm{C} & -1.57133 & 0.05144 & 0.14186 \\ \mathrm{C} & -0.94966 & 1.32929 & 0.36645 \\ \mathrm{C} & -0.50458 & -0.98569 & 0.40952 \\ \mathrm{C} & 0.66309 & -0.31876 & -0.28727 \\ \mathrm{C} & 1.96115 & -0.18379 & 0.27732 \\ \mathrm{C} & 3.07404 & 0.22493 & -0.39890 \\ \mathrm{H} & -3.40027 & 0.65412 & -0.77499 \\ \mathrm{H} & -3.10547 & -1.17542 & -0.69174 \\ \mathrm{H} & -1.34389 & 2.23987 & -0.08319 \\ \mathrm{H} & -0.29227 & 1.46687 & 1.21858 \\ \mathrm{H} & -0.74430 & -1.98576 & 0.02443 \\ \mathrm{H} & -0.29750 & -1.08624 & 1.48457 \\ \mathrm{H} & 0.55429 & -0.17557 & -1.36090 \\ \mathrm{H} & 2.06223 & -0.41828 & 1.33898 \\ \mathrm{H} & 3.03628 & 0.47231 & -1.45755 \\ \mathrm{H} & 4.03557 & 0.32020 & 0.09643\end{array}$

$\mathrm{E}(\mathrm{UB}+\mathrm{HF}-\mathrm{LYP})=-272.640041632$

$\mathrm{S} * * 2=0.5090$

1 imaginary frequency

$383 \mathrm{~K}, 1.00 \mathrm{~atm}$, freq's scaled by 1.0000

Zero-point correction $=0.143668$

Thermal correction to $\mathrm{U}=0.155449$

Thermal correction to $\mathrm{H}=0.156661$

Thermal correction to $\mathrm{G}=0.100486$

$\mathrm{E}(\operatorname{CASPT} 2(6,6) / 6-31 \mathrm{G}(\mathrm{d}))=-271.66748147$

$(6,6) \mathrm{CASSCF} / 6-31+\mathrm{G} *$ :

$\begin{array}{lrrr}\text { C } & -2.77365 & -0.27049 & -0.44463 \\ \mathrm{C} & -1.58702 & 0.03610 & 0.12131 \\ \mathrm{C} & -1.05069 & 1.37994 & 0.29119 \\ \mathrm{C} & -0.47549 & -0.93102 & 0.42787 \\ \mathrm{C} & 0.69809 & -0.29174 & -0.28214 \\ \mathrm{C} & 1.99864 & -0.16388 & 0.29617 \\ \mathrm{C} & 3.12346 & 0.19832 & -0.38769 \\ \mathrm{H} & -3.46672 & 0.49613 & -0.74401 \\ \mathrm{H} & -3.06496 & -1.29073 & -0.62042 \\ \mathrm{H} & -1.50869 & 2.22514 & -0.19424 \\ \mathrm{H} & -0.46414 & 1.59748 & 1.16435 \\ \mathrm{H} & -0.67747 & -1.94563 & 0.09502 \\ \mathrm{H} & -0.28482 & -0.97245 & 1.49836 \\ \mathrm{H} & 0.61363 & -0.20997 & -1.35272 \\ \mathrm{H} & 2.08435 & -0.34620 & 1.35625 \\ \mathrm{H} & 3.09899 & 0.39830 & -1.44450 \\ \mathrm{H} & 4.06989 & 0.30452 & 0.10939\end{array}$

$\mathrm{E}(\operatorname{CASSCF}(6,6) / 6-31+\mathrm{G}(\mathrm{d}))=-270.8426023207$

$\mathrm{E}(\operatorname{CASPT} 2(6,6) / 6-31 \mathrm{G}(\mathrm{d}))=-271.6655011391$
TS-1-4

$\mathrm{UB} 3 \mathrm{LYP} / 6-31+\mathrm{G}(\mathrm{d})$ :

$\begin{array}{lrrr}\mathrm{C} & 2.22134 & -1.03941 & -0.26976 \\ \mathrm{C} & 1.45021 & 0.04254 & -0.00642 \\ \mathrm{C} & 1.07883 & 1.06914 & -0.94332 \\ \mathrm{C} & 0.56155 & 0.22237 & 1.20561 \\ \mathrm{C} & -0.76105 & 0.53061 & 0.51715 \\ \mathrm{C} & -1.58154 & -0.49164 & -0.03594 \\ \mathrm{C} & -2.88085 & -0.33089 & -0.41536 \\ \mathrm{H} & 2.38314 & -1.81686 & 0.47188 \\ \mathrm{H} & 2.70213 & -1.16808 & -1.23666 \\ \mathrm{H} & 0.52402 & -0.65914 & 1.85729 \\ \mathrm{H} & 0.87803 & 1.07444 & 1.82029 \\ \mathrm{H} & 0.87051 & 2.07400 & -0.58982 \\ \mathrm{H} & 1.29509 & 0.97314 & -2.00637 \\ \mathrm{H} & -1.21732 & 1.50587 & 0.67991 \\ \mathrm{H} & -1.12210 & -1.47368 & -0.16171 \\ \mathrm{H} & -3.45238 & -1.14903 & -0.84313 \\ \mathrm{H} & -3.39207 & 0.62306 & -0.30337\end{array}$

$\mathrm{E}(\mathrm{UB}+\mathrm{HF}-\mathrm{LYP})=-272.637131664$

$\mathrm{S} * * 2=0.5254$

1 imaginary frequency

$383 \mathrm{~K}, 1.00 \mathrm{~atm}$, freq's scaled by 1.0000

Zero-point correction $=0.143496$

Thermal correction to $\mathrm{U}=0.155374$

Thermal correction to $\mathrm{H}=0.156587$

Thermal correction to $\mathrm{G}=0.100085$

$\mathrm{E}(\operatorname{CASPT} 2(6,6) / 6-31 \mathrm{G}(\mathrm{d}))=-271.6652012453$

$(6,6) \mathrm{CASSCF} / 6-31+\mathrm{G} *$ :

$\begin{array}{lrrr}\mathrm{C} & 2.26681 & -0.95434 & -0.45176 \\ \mathrm{C} & 1.46210 & 0.02528 & 0.01297 \\ \mathrm{C} & 1.15753 & 1.26679 & -0.68457 \\ \mathrm{C} & 0.52956 & -0.08112 & 1.19156 \\ \mathrm{C} & -0.79349 & 0.39967 & 0.61796 \\ \mathrm{C} & -1.62938 & -0.46395 & -0.15946 \\ \mathrm{C} & -2.92712 & -0.20123 & -0.48554 \\ \mathrm{H} & 2.38427 & -1.88336 & 0.07732 \\ \mathrm{H} & 2.81201 & -0.84153 & -1.37254 \\ \mathrm{H} & 0.47344 & -1.08504 & 1.60189 \\ \mathrm{H} & 0.84448 & 0.57863 & 1.99529 \\ \mathrm{H} & 1.00741 & 2.16222 & -0.11041 \\ \mathrm{H} & 1.43690 & 1.40643 & -1.71502 \\ \mathrm{H} & -1.24916 & 1.27665 & 1.04541 \\ \mathrm{H} & -1.18552 & -1.37755 & -0.52268 \\ \mathrm{H} & -3.49909 & -0.87858 & -1.09237 \\ \mathrm{H} & -3.42078 & 0.69556 & -0.15377\end{array}$

$\mathrm{E}(\operatorname{CASSCF}(6,6) / 6-31+\mathrm{G}(\mathrm{d}))=-270.8400062769$

$\mathrm{E}(\operatorname{CASPT} 2(6,6) / 6-31 \mathrm{G}(\mathrm{d}))=-271.6631201524$ 
UB3LYP / 6-31+G (d) :

$\begin{array}{lrrr}\mathrm{C} & 3.21965 & -0.48992 & -0.38895 \\ \mathrm{C} & 2.01102 & 0.14552 & -0.63260 \\ \mathrm{C} & 0.93277 & 0.18834 & 0.24660 \\ \mathrm{C} & -0.35729 & 0.90788 & -0.02412 \\ \mathrm{C} & -1.58719 & 0.02730 & 0.20717 \\ \mathrm{C} & -1.76047 & -1.09049 & -0.61059 \\ \mathrm{C} & -2.48202 & 0.34530 & 1.21780 \\ \mathrm{H} & -2.34569 & 1.22071 & 1.84597 \\ \mathrm{H} & -3.35083 & -0.27735 & 1.41455 \\ \mathrm{H} & -0.43672 & 1.79489 & 0.62313 \\ \mathrm{H} & -0.35921 & 1.27993 & -1.05754 \\ \mathrm{H} & 1.89319 & 0.65418 & -1.59191 \\ \mathrm{H} & 3.40404 & -1.01301 & 0.54632 \\ \mathrm{H} & 1.02136 & -0.31577 & 1.20958 \\ \mathrm{H} & -1.04433 & -1.34047 & -1.38739 \\ \mathrm{H} & -2.62166 & -1.74277 & -0.49420 \\ \mathrm{H} & 4.01825 & -0.48737 & -1.12383\end{array}$

$\mathrm{E}(\mathrm{UB}+\mathrm{HF}-\mathrm{LYP})=-272.654553441$ $\mathrm{S} * * 2=1.0321$

$383 \mathrm{~K}, 1.00 \mathrm{~atm}$, freq's scaled by 1.0000

Zero-point correction $=0.142791$

Thermal correction to $\mathrm{U}=0.155866$

Thermal correction to $\mathrm{H}=0.157079$

Thermal correction to $\mathrm{G}=0.096919$

$\operatorname{E}(\operatorname{CASPT} 2(6,6) / 6-31 G(d))=-271.675484039$

\section{$(6,6)$ CASSCF $/ 6-31+G *$ :}

$\begin{array}{lrrr}\mathrm{C} & 3.26636 & 0.14487 & -0.43526 \\ \mathrm{C} & 2.11057 & -0.00960 & 0.32253 \\ \mathrm{C} & 0.86459 & -0.36655 & -0.18962 \\ \mathrm{C} & -0.36273 & -0.59013 & 0.65403 \\ \mathrm{C} & -1.60659 & 0.06232 & 0.06749 \\ \mathrm{C} & -1.66126 & 1.46176 & 0.01969 \\ \mathrm{C} & -2.64270 & -0.72396 & -0.41459 \\ \mathrm{H} & -2.60216 & -1.79669 & -0.37303 \\ \mathrm{H} & -3.51954 & -0.28122 & -0.85146 \\ \mathrm{H} & -0.54303 & -1.65732 & 0.76290 \\ \mathrm{H} & -0.18856 & -0.20703 & 1.65587 \\ \mathrm{H} & 2.18356 & 0.16647 & 1.38485 \\ \mathrm{H} & 3.26332 & -0.01609 & -1.49833 \\ \mathrm{H} & 0.77157 & -0.55307 & -1.24734 \\ \mathrm{H} & -0.85274 & 2.06596 & 0.38666 \\ \mathrm{H} & -2.51704 & 1.97131 & -0.38397 \\ \mathrm{H} & 4.19514 & 0.43541 & 0.01819\end{array}$

$\mathrm{E}(\operatorname{CASSCF}(6,6) / 6-31+\mathrm{G}(\mathrm{d}))=-270.854822994$ $\mathrm{E}(\operatorname{CASPT} 2(6,6) / 6-31 \mathrm{G}(\mathrm{d}))=-271.674705921$
UB3LYP / 6-31+G(d) :

$\begin{array}{lrrr}\mathrm{C} & 2.94274 & -0.63011 & 0.00000 \\ \mathrm{C} & 1.57617 & -0.38728 & 0.00000 \\ \mathrm{C} & 0.99973 & 0.87817 & 0.00000 \\ \mathrm{C} & -0.47078 & 1.20520 & -0.00000 \\ \mathrm{C} & -1.41534 & 0.01194 & 0.00000 \\ \mathrm{C} & -1.83175 & -0.51965 & 1.21680 \\ \mathrm{C} & -1.83175 & -0.51965 & -1.21680 \\ \mathrm{H} & -1.50306 & -0.09963 & 2.16280 \\ \mathrm{H} & -2.50032 & -1.37558 & 1.25699 \\ \mathrm{H} & -0.69114 & 1.84068 & 0.87269 \\ \mathrm{H} & -0.69114 & 1.84068 & -0.87270 \\ \mathrm{H} & 0.90860 & -1.24821 & 0.00000 \\ \mathrm{H} & 3.66283 & 0.18498 & 0.00000 \\ \mathrm{H} & 1.66667 & 1.74193 & 0.00000 \\ \mathrm{H} & -1.50306 & -0.09963 & -2.16280 \\ \mathrm{H} & -2.50032 & -1.37558 & -1.25699 \\ \mathrm{H} & 3.33675 & -1.64135 & 0.00000\end{array}$

$E(\mathrm{UB}+\mathrm{HF}-\mathrm{LYP})=-272.653153637$

$\mathrm{S} * * 2=1.0307$

$383 \mathrm{~K}, 1.00 \mathrm{~atm}$, freq's scaled by 1.0000

Zero-point correction $=0.142690$

Thermal correction to $\mathrm{U}=0.155791$

Thermal correction to $\mathrm{H}=0.157004$

Thermal correction to $\mathrm{G}=0.096756$

$\mathrm{E}(\operatorname{CASPT} 2(6,6) / 6-31 \mathrm{G}(\mathrm{d}))=-271.6749346287$

\section{$(6,6) \mathrm{CASSCF} / 6-31+\mathrm{G} *$ :}

$\begin{array}{lrrr}\mathrm{C} & 0.08040 & 0.00000 & -0.08922 \\ \mathrm{C} & -0.02681 & 0.00000 & 1.29943 \\ \mathrm{C} & 1.06329 & 0.00000 & 2.16501 \\ \mathrm{C} & 1.03009 & 0.00000 & 3.67450 \\ \mathrm{C} & -0.34969 & 0.00000 & 4.30739 \\ \mathrm{C} & -0.96915 & -1.21708 & 4.58997 \\ \mathrm{C} & -0.96915 & 1.21708 & 4.58997 \\ \mathrm{H} & -0.49075 & -2.15379 & 4.37165 \\ \mathrm{H} & -1.94790 & -1.25335 & 5.03281 \\ \mathrm{H} & 1.58470 & -0.86550 & 4.03141 \\ \mathrm{H} & 1.58470 & 0.86550 & 4.03141 \\ \mathrm{H} & -1.01635 & 0.00000 & 1.72253 \\ \mathrm{H} & 1.03837 & -0.00000 & -0.57766 \\ \mathrm{H} & 2.05041 & -0.00000 & 1.73023 \\ \mathrm{H} & -0.49075 & 2.15379 & 4.37165 \\ \mathrm{H} & -1.94790 & 1.25335 & 5.03281 \\ \mathrm{H} & -0.79259 & 0.00000 & -0.71408\end{array}$

$\mathrm{E}(\operatorname{CASSCF}(6,6) / 6-31+\mathrm{G}(\mathrm{d}))=-270.8522794616$ $\mathrm{E}(\mathrm{CASPT} 2(6,6) / 6-31 \mathrm{G}(\mathrm{d}))=-271.674152436$ 
TS-1-5

UB3LYP / 6-31+G (d) :

$\begin{array}{lrrr}\mathrm{C} & -2.74523 & -0.11894 & -0.54730 \\ \mathrm{C} & -1.98756 & 0.17373 & 0.55113 \\ \mathrm{C} & -0.61506 & -0.14413 & 0.74983 \\ \mathrm{C} & 0.26528 & -0.92224 & -0.20554 \\ \mathrm{C} & 1.46131 & 0.00417 & -0.22757 \\ \mathrm{C} & 0.94068 & 1.34077 & -0.29963 \\ \mathrm{C} & 2.73861 & -0.34389 & 0.06383 \\ \mathrm{H} & 3.02882 & -1.38269 & 0.19486 \\ \mathrm{H} & 3.51684 & 0.40698 & 0.17739 \\ \mathrm{H} & 0.49457 & -1.94124 & 0.13148 \\ \mathrm{H} & -0.19671 & -0.99763 & -1.19754 \\ \mathrm{H} & -2.46703 & 0.72280 & 1.36213 \\ \mathrm{H} & -2.35400 & -0.66981 & -1.39803 \\ \mathrm{H} & -0.20757 & -0.01205 & 1.74866 \\ \mathrm{H} & 1.54172 & 2.19804 & 0.00153 \\ \mathrm{H} & 0.08168 & 1.55494 & -0.92664 \\ \mathrm{H} & -3.78651 & 0.18384 & -0.60235\end{array}$

$\mathrm{E}(\mathrm{UB}+\mathrm{HF}-\mathrm{LYP})=-272.640350520$

$\mathrm{S} * * 2=0.4936$

$383 \mathrm{~K}, 1.00 \mathrm{~atm}$, freq's scaled by 1.0000

Zero-point correction $=0.144053$

Thermal correction to $\mathrm{U}=0.155732$

Thermal correction to $\mathrm{H}=0.156945$

Thermal correction to $\mathrm{G}=0.100972$

$\mathrm{E}(\operatorname{CASPT} 2(6,6) / 6-31 \mathrm{G}(\mathrm{d}))=-271.6676960499$

\section{$(6,6) \mathrm{CASSCF} / 6-31+\mathrm{G} *$ :}

$\begin{array}{lrrr}\mathrm{C} & -2.77912 & -0.11366 & -0.56647 \\ \mathrm{C} & -2.03712 & 0.15902 & 0.54723 \\ \mathrm{C} & -0.64920 & -0.12327 & 0.74633 \\ \mathrm{C} & 0.24950 & -0.87450 & -0.21210 \\ \mathrm{C} & 1.48150 & -0.00793 & -0.20513 \\ \mathrm{C} & 1.03989 & 1.37720 & -0.26757 \\ \mathrm{C} & 2.74024 & -0.41966 & 0.05773 \\ \mathrm{H} & 2.97660 & -1.46181 & 0.17924 \\ \mathrm{H} & 3.54967 & 0.28196 & 0.15935 \\ \mathrm{H} & 0.43621 & -1.89986 & 0.09466 \\ \mathrm{H} & -0.18355 & -0.90657 & -1.20677 \\ \mathrm{H} & -2.53334 & 0.65829 & 1.36381 \\ \mathrm{H} & -2.37393 & -0.61187 & -1.42760 \\ \mathrm{H} & -0.27068 & -0.02637 & 1.74841 \\ \mathrm{H} & 1.68287 & 2.17815 & 0.05651 \\ \mathrm{H} & 0.25862 & 1.64388 & -0.95464 \\ \mathrm{H} & -3.81672 & 0.16114 & -0.61317\end{array}$

$\mathrm{E}(\operatorname{CASSCF}(6,6) / 6-31+\mathrm{G}(\mathrm{d}))=-270.8428653438$ $\mathrm{E}(\operatorname{CASPT} 2(6,6) / 6-31 \mathrm{G}(\mathrm{d}))=-271.6655989459$
TS-1-6

$\mathrm{UB} 3 L Y P / 6-31+G(d)$ :

$\begin{array}{lrrr}\mathrm{C} & 1.97775 & 1.04620 & -0.19573 \\ \mathrm{C} & 1.76105 & -0.29246 & -0.36314 \\ \mathrm{C} & 0.73507 & -1.05412 & 0.25784 \\ \mathrm{C} & -0.31136 & -0.43850 & 1.17092 \\ \mathrm{C} & -1.37717 & -0.90288 & -0.89466 \\ \mathrm{C} & -1.23873 & 0.11026 & 0.10260 \\ \mathrm{C} & -1.56503 & 1.41963 & -0.04874 \\ \mathrm{H} & -1.44968 & -1.94645 & -0.60730 \\ \mathrm{H} & -1.70714 & -0.66195 & -1.90421 \\ \mathrm{H} & -0.79390 & -1.21052 & 1.78208 \\ \mathrm{H} & 0.08034 & 0.32720 & 1.85007 \\ \mathrm{H} & 2.41330 & -0.83457 & -1.04801 \\ \mathrm{H} & 1.39492 & 1.64796 & 0.49331 \\ \mathrm{H} & 0.82251 & -2.13668 & 0.22860 \\ \mathrm{H} & -1.35535 & 2.15181 & 0.72600 \\ \mathrm{H} & -2.05742 & 1.77739 & -0.94964 \\ \mathrm{H} & 2.76295 & 1.55699 & -0.74542\end{array}$

$\mathrm{E}(\mathrm{UB}+\mathrm{HF}-\mathrm{LYP})=-272.638293669$

$\mathrm{S} * * 2=0.4212$

1 imaginary frequency

$383 \mathrm{~K}, 1.00 \mathrm{~atm}$, freq's scaled by 1.0000

Zero-point correction $=0.144287$

Thermal correction to $\mathrm{U}=0.155833$

Thermal correction to $\mathrm{H}=0.157046$

Thermal correction to $\mathrm{G}=0.101642$

$\mathrm{E}(\operatorname{CASPT} 2(6,6) / 6-31 \mathrm{G}(\mathrm{d}))=-271.6670355328$

$(6,6) \mathrm{CASSCF} / 6-31+\mathrm{G}^{*}$ :

$\begin{array}{lrrr}\mathrm{C} & 2.04407 & 1.08654 & -0.27264 \\ \mathrm{C} & 1.86370 & -0.26615 & -0.31241 \\ \mathrm{C} & 0.80496 & -0.99982 & 0.30956 \\ \mathrm{C} & -0.28301 & -0.35019 & 1.14668 \\ \mathrm{C} & -1.36247 & -0.98120 & -0.89529 \\ \mathrm{C} & -1.27460 & 0.07978 & 0.09420 \\ \mathrm{C} & -1.78492 & 1.32180 & -0.05047 \\ \mathrm{H} & -1.44289 & -1.99937 & -0.56245 \\ \mathrm{H} & -1.72317 & -0.78033 & -1.88989 \\ \mathrm{H} & -0.71835 & -1.08432 & 1.81865 \\ \mathrm{H} & 0.07614 & 0.47109 & 1.75763 \\ \mathrm{H} & 2.57191 & -0.85031 & -0.87746 \\ \mathrm{H} & 1.38892 & 1.74095 & 0.27053 \\ \mathrm{H} & 0.93169 & -2.06438 & 0.38663 \\ \mathrm{H} & -1.61480 & 2.08930 & 0.68373 \\ \mathrm{H} & -2.37767 & 1.58694 & -0.90841 \\ \mathrm{H} & 2.86189 & 1.54599 & -0.79674\end{array}$

$\mathrm{E}(\operatorname{CASSCF}(6,6) / 6-31+\mathrm{G}(\mathrm{d}))=-270.8407220039$

$\mathrm{E}(\operatorname{CASPT} 2(6,6) / 6-31 \mathrm{G}(\mathrm{d}))=-271.6641672792$ 
UB3LYP / 6-31+G(d) :

$\begin{array}{lrrr}\text { C } & -2.98648 & -0.00019 & -0.53515 \\ \mathrm{C} & -2.21894 & 0.00056 & 0.61540 \\ \mathrm{C} & -0.82162 & -0.00047 & 0.67524 \\ \mathrm{C} & 0.08091 & -0.00259 & -0.53127 \\ \mathrm{C} & 1.55532 & 0.00007 & -0.15740 \\ \mathrm{C} & 2.20589 & -1.21598 & 0.02688 \\ \mathrm{C} & 2.20174 & 1.21867 & 0.02501 \\ \mathrm{H} & 1.68617 & 2.16281 & -0.12412 \\ \mathrm{H} & 3.24638 & 1.26247 & 0.32131 \\ \mathrm{H} & -0.13680 & -0.88053 & -1.15922 \\ \mathrm{H} & -0.13879 & 0.87159 & -1.16372 \\ \mathrm{H} & -2.74581 & 0.00205 & 1.57003 \\ \mathrm{H} & -2.54067 & -0.00173 & -1.52594 \\ \mathrm{H} & -0.34477 & 0.00001 & 1.65247 \\ \mathrm{H} & 1.69360 & -2.16212 & -0.12079 \\ \mathrm{H} & 3.25066 & -1.25572 & 0.32332 \\ \mathrm{H} & -4.07083 & 0.00072 & -0.48562\end{array}$

$\mathrm{E}(\mathrm{UB}+\mathrm{HF}-\mathrm{LYP})=-272.653399979$

$\mathrm{S} * * 2=1.0219$

1 imaginary frequency

$383 \mathrm{~K}, 1.00 \mathrm{~atm}$, freq's scaled by 1.0000

Zero-point correction $=0.142833$

Thermal correction to $\mathrm{U}=0.154722$

Thermal correction to $\mathrm{H}=0.155935$

Thermal correction to $\mathrm{G}=0.099263$

$\mathrm{E}(\operatorname{CASPT} 2(6,6) / 6-31 \mathrm{G}(\mathrm{d}))=-271.6744562937$

\section{$(6,6) \mathrm{CASSCF} / 6-31+\mathrm{G} *$}

$\begin{array}{lrrr}\text { C } & -2.98751 & -0.00019 & -0.53495 \\ \mathrm{C} & -2.21889 & 0.00053 & 0.61779 \\ \mathrm{C} & -0.81817 & -0.00035 & 0.67485 \\ \mathrm{C} & 0.08176 & -0.00251 & -0.53712 \\ \mathrm{C} & 1.55168 & 0.00003 & -0.16093 \\ \mathrm{C} & 2.20571 & -1.21663 & 0.02881 \\ \mathrm{C} & 2.20168 & 1.21924 & 0.02699 \\ \mathrm{H} & 1.69304 & 2.15394 & -0.12001 \\ \mathrm{H} & 3.23394 & 1.25968 & 0.32370 \\ \mathrm{H} & -0.13165 & -0.87295 & -1.15325 \\ \mathrm{H} & -0.13349 & 0.86427 & -1.15771 \\ \mathrm{H} & -2.74072 & 0.00182 & 1.56093 \\ \mathrm{H} & -2.54856 & -0.00156 & -1.51541 \\ \mathrm{H} & -0.34841 & -0.00007 & 1.64160 \\ \mathrm{H} & 1.70023 & -2.15326 & -0.11675 \\ \mathrm{H} & 3.23810 & -1.25315 & 0.32560 \\ \mathrm{H} & -4.05999 & 0.00059 & -0.48144\end{array}$

$\mathrm{E}(\operatorname{CASSCF}(6,6) / 6-31+\mathrm{G}(\mathrm{d}))=-270.8538450776$ $\mathrm{E}(\operatorname{CASPT} 2(6,6) / 6-31 \mathrm{G}(\mathrm{d}))=-271.6737647213$
UB3LYP / 6-31+G(d) :

$\begin{array}{lrrr}\mathrm{C} & 2.37623 & 0.92519 & -0.26691 \\ \mathrm{C} & 2.03784 & -0.41664 & -0.33962 \\ \mathrm{C} & 0.90606 & -1.02820 & 0.20250 \\ \mathrm{C} & -0.18479 & -0.32465 & 0.96589 \\ \mathrm{C} & -1.35822 & 0.11154 & 0.07749 \\ \mathrm{C} & -2.09058 & -0.86667 & -0.59543 \\ \mathrm{C} & -1.66014 & 1.46036 & -0.04106 \\ \mathrm{H} & -1.10384 & 2.22098 & 0.49842 \\ \mathrm{H} & -2.46644 & 1.79969 & -0.68617 \\ \mathrm{H} & -0.56722 & -1.00009 & 1.74308 \\ \mathrm{H} & 0.21440 & 0.55282 & 1.48595 \\ \mathrm{H} & 2.72494 & -1.06980 & -0.87873 \\ \mathrm{H} & 1.75589 & 1.65387 & 0.24558 \\ \mathrm{H} & 0.78386 & -2.09700 & 0.03979 \\ \mathrm{H} & -1.85384 & -1.92250 & -0.50530 \\ \mathrm{H} & -2.93137 & -0.59923 & -1.22947 \\ \mathrm{H} & 3.28515 & 1.29567 & -0.73028\end{array}$

$\mathrm{E}(\mathrm{UB}+\mathrm{HF}-\mathrm{LYP})=-272.651254005$

$\mathrm{S} * * 2=1.0330$

$383 \mathrm{~K}, 1.00 \mathrm{~atm}$, freq's scaled by 1.0000

Zero-point correction $=0.143168$

Thermal correction to $\mathrm{U}=0.156160$

Thermal correction to $\mathrm{H}=0.157373$

Thermal correction to $\mathrm{G}=0.096760$

$\mathrm{E}(\operatorname{CASPT} 2(6,6) / 6-31 \mathrm{G}(\mathrm{d}))=-271.6726770071$

\section{$(6,6)$ CASSCF $/ 6-31+G *$ :}

$\begin{array}{lrrr}\mathrm{C} & 2.25346 & 1.00630 & -0.33301 \\ \mathrm{C} & 2.03123 & -0.36444 & -0.31762 \\ \mathrm{C} & 0.95130 & -1.02790 & 0.27199 \\ \mathrm{C} & -0.19187 & -0.35819 & 0.99513 \\ \mathrm{C} & -1.32750 & 0.07969 & 0.07387 \\ \mathrm{C} & -1.96155 & -0.88635 & -0.71814 \\ \mathrm{C} & -1.71703 & 1.41082 & 0.03810 \\ \mathrm{H} & -1.24915 & 2.15360 & 0.65727 \\ \mathrm{H} & -2.50274 & 1.74169 & -0.61676 \\ \mathrm{H} & -0.58782 & -1.05025 & 1.73293 \\ \mathrm{H} & 0.16791 & 0.50061 & 1.55067 \\ \mathrm{H} & 2.76218 & -0.98388 & -0.81195 \\ \mathrm{H} & 1.58179 & 1.70335 & 0.13038 \\ \mathrm{H} & 0.91890 & -2.09956 & 0.18477 \\ \mathrm{H} & -1.66630 & -1.91849 & -0.69411 \\ \mathrm{H} & -2.77092 & -0.62112 & -1.37333 \\ \mathrm{H} & 3.11800 & 1.41454 & -0.82177\end{array}$

$\mathrm{E}(\operatorname{CASSCF}(6,6) / 6-31+\mathrm{G}(\mathrm{d}))=-270.8516167948$ $\mathrm{E}(\operatorname{CASPT} 2(6,6) / 6-31 \mathrm{G}(\mathrm{d}))=-271.6722618787$ 
TS-6-2-x

UB3LYP / 6-31+G (d) :

$\begin{array}{rrrr}\text { C } & -0.84999 & 1.53815 & 0.22268 \\ \mathrm{C} & 1.82536 & 0.96122 & -0.11748 \\ \mathrm{C} & 1.70572 & -0.37642 & -0.51911 \\ \mathrm{C} & 0.81929 & -1.27361 & 0.04353 \\ \mathrm{C} & -0.28548 & -0.81718 & 0.96013 \\ \mathrm{C} & -1.14426 & 0.16589 & 0.14900 \\ \mathrm{C} & -2.03851 & -0.36155 & -0.74896 \\ \mathrm{H} & -2.19396 & -1.43373 & -0.83260 \\ \mathrm{H} & -2.64614 & 0.27682 & -1.38533 \\ \mathrm{H} & -0.88343 & -1.66982 & 1.29659 \\ \mathrm{H} & 0.09861 & -0.31647 & 1.86070 \\ \mathrm{H} & 2.29487 & -0.70763 & -1.37504 \\ \mathrm{H} & 1.58317 & 1.27008 & 0.88990 \\ \mathrm{H} & 0.81748 & -2.30833 & -0.29031 \\ \mathrm{H} & -0.41778 & 1.97674 & 1.11460 \\ \mathrm{H} & -1.25453 & 2.22807 & -0.51308 \\ \mathrm{H} & 2.40901 & 1.66522 & -0.70415\end{array}$

$\mathrm{E}(\mathrm{UB}+\mathrm{HF}-\mathrm{LYP})=-272.644286520$

$\mathrm{S} * * 2=0.5766$

1 imaginary frequency

$383 \mathrm{~K}, 1.00 \mathrm{~atm}$, freq's scaled by 1.0000

Zero-point correction $=0.144252$

Thermal correction to $\mathrm{U}=0.155564$

Thermal correction to $\mathrm{H}=0.156776$

Thermal correction to $\mathrm{G}=0.102332$

$\mathrm{E}(\operatorname{CASPT} 2(6,6) / 6-31 \mathrm{G}(\mathrm{d}))=-271.6729352347$

$(6,6)$ CASSCF $/ 6-31-G^{*}:$

$\begin{array}{lrrr}\mathrm{C} & -0.88333 & 1.53447 & 0.21197 \\ \mathrm{C} & 1.84209 & 0.98881 & -0.12165 \\ \mathrm{C} & 1.74522 & -0.37410 & -0.50483 \\ \mathrm{C} & 0.85262 & -1.26301 & 0.04147 \\ \mathrm{C} & -0.27854 & -0.81695 & 0.93865 \\ \mathrm{C} & -1.16431 & 0.14279 & 0.14806 \\ \mathrm{C} & -2.07771 & -0.37444 & -0.73161 \\ \mathrm{H} & -2.24068 & -1.43405 & -0.81493 \\ \mathrm{H} & -2.67873 & 0.26226 & -1.35599 \\ \mathrm{H} & -0.85292 & -1.67553 & 1.26658 \\ \mathrm{H} & 0.09178 & -0.32708 & 1.83560 \\ \mathrm{H} & 2.36714 & -0.71088 & -1.31830 \\ \mathrm{H} & 1.60915 & 1.29774 & 0.87526 \\ \mathrm{H} & 0.85353 & -2.28356 & -0.29868 \\ \mathrm{H} & -0.46640 & 1.97127 & 1.09797 \\ \mathrm{H} & -1.34811 & 2.20920 & -0.48433 \\ \mathrm{H} & 2.44904 & 1.66525 & -0.69557\end{array}$

$\mathrm{E}(\operatorname{CASSCF}(6,6) / 6-31+\mathrm{G}(\mathrm{d}))=-270.8434384118$

$\mathrm{E}(\operatorname{CASPT} 2(6,6) / 6-31 \mathrm{G}(\mathrm{d}))=-271.6708520249$
TS-6-2-n

$\mathrm{UB} 3 \mathrm{LYP} / 6-31+\mathrm{G}(\mathrm{d})$ :

$\begin{array}{lrrr}\mathrm{C} & 1.47075 & -1.12690 & -0.71158 \\ \mathrm{C} & 1.94031 & 0.01070 & -0.02713 \\ \mathrm{C} & 1.18018 & 1.12208 & 0.26242 \\ \mathrm{C} & -0.28001 & 1.24783 & -0.06779 \\ \mathrm{C} & -1.14550 & -0.00780 & 0.14883 \\ \mathrm{C} & -2.38608 & -0.03728 & -0.44267 \\ \mathrm{C} & -0.66277 & -1.09664 & 0.90323 \\ \mathrm{H} & 0.18189 & -1.00862 & 1.57108 \\ \mathrm{H} & -1.26276 & -2.00040 & 0.97698 \\ \mathrm{H} & -0.70834 & 2.07497 & 0.51310 \\ \mathrm{H} & -0.40495 & 1.53818 & -1.12521 \\ \mathrm{H} & 2.95418 & -0.02617 & 0.37307 \\ \mathrm{H} & 0.61877 & -1.09106 & -1.37744 \\ \mathrm{H} & 1.64773 & 1.95320 & 0.78534 \\ \mathrm{H} & -2.74269 & 0.78396 & -1.05942 \\ \mathrm{H} & -3.06608 & -0.87107 & -0.28952 \\ \mathrm{H} & 2.08101 & -2.02500 & -0.75984\end{array}$

$\mathrm{E}(\mathrm{UB}+\mathrm{HF}-\mathrm{LYP})=-272.644661348$

$\mathrm{S} * * 2=0.6696$

1 imaginary frequency

$383 \mathrm{~K}, 1.00 \mathrm{~atm}$, freq's scaled by 1.0000

Zero-point correction $=0.143949$

Thermal correction to $\mathrm{U}=0.155362$

Thermal correction to $\mathrm{H}=0.156575$

Thermal correction to $\mathrm{G}=0.101607$

$\mathrm{E}(\operatorname{CASPT} 2(6,6) / 6-31 \mathrm{G}(\mathrm{d}))=-271.6723644291$

$(6,6) \mathrm{CASSCF} / 6-31-\mathrm{G}^{*}:$

$\begin{array}{lrrr}\mathrm{C} & 1.48141 & -1.15384 & -0.70738 \\ \mathrm{C} & 1.94833 & 0.00904 & -0.03529 \\ \mathrm{C} & 1.18367 & 1.10838 & 0.26013 \\ \mathrm{C} & -0.28351 & 1.25255 & -0.06110 \\ \mathrm{C} & -1.15266 & 0.00929 & 0.14638 \\ \mathrm{C} & -2.36481 & -0.05059 & -0.49526 \\ \mathrm{C} & -0.70119 & -1.06863 & 0.95531 \\ \mathrm{H} & 0.11530 & -0.96370 & 1.63777 \\ \mathrm{H} & -1.32506 & -1.93757 & 1.06631 \\ \mathrm{H} & -0.68833 & 2.05833 & 0.54455 \\ \mathrm{H} & -0.40041 & 1.57301 & -1.09499 \\ \mathrm{H} & 2.96002 & -0.01033 & 0.33670 \\ \mathrm{H} & 0.63599 & -1.12923 & -1.36266 \\ \mathrm{H} & 1.65264 & 1.93393 & 0.76649 \\ \mathrm{H} & -2.70062 & 0.74140 & -1.14077 \\ \mathrm{H} & -3.03134 & -0.88289 & -0.35689 \\ \mathrm{H} & 2.11445 & -2.02010 & -0.77329\end{array}$

$\mathrm{E}(\operatorname{CASSCF}(6,6) / 6-31+\mathrm{G}(\mathrm{d}))=-270.8440719592$

$\mathrm{E}(\operatorname{CASPT} 2(6,6) / 6-31 \mathrm{G}(\mathrm{d}))=-271.6704541268$ 
TS-5-6

UB3LYP / 6-31+G (d) :

$\begin{array}{lrrr}\mathrm{C} & 2.53478 & 0.83469 & -0.17660 \\ \mathrm{C} & 2.06501 & -0.45761 & -0.35752 \\ \mathrm{C} & 0.86861 & -0.99778 & 0.11466 \\ \mathrm{C} & -0.17550 & -0.26559 & 0.91440 \\ \mathrm{C} & -1.39883 & 0.13675 & 0.07990 \\ \mathrm{C} & -2.21981 & -0.86457 & -0.43854 \\ \mathrm{C} & -1.65850 & 1.48156 & -0.14497 \\ \mathrm{H} & -1.02927 & 2.26319 & 0.26968 \\ \mathrm{H} & -2.50365 & 1.79473 & -0.75253 \\ \mathrm{H} & -0.51350 & -0.91485 & 1.73472 \\ \mathrm{H} & 0.25115 & 0.62783 & 1.38082 \\ \mathrm{H} & 2.69833 & -1.13247 & -0.93458 \\ \mathrm{H} & 1.98153 & 1.58395 & 0.38134 \\ \mathrm{H} & 0.65062 & -2.03399 & -0.13643 \\ \mathrm{H} & -2.02250 & -1.91732 & -0.25888 \\ \mathrm{H} & -3.09330 & -0.61898 & -1.03610 \\ \mathrm{H} & 3.48799 & 1.14210 & -0.59478\end{array}$

$\mathrm{E}(\mathrm{UB}+\mathrm{HF}-\mathrm{LYP})=-272.651223588$

$\mathrm{S} * * 2=1.0457$

1 imaginary frequency

$383 \mathrm{~K}, 1.00 \mathrm{~atm}$, freq's scaled by 1.0000

Zero-point correction $=0.142986$

Thermal correction to $\mathrm{U}=0.154871$

Thermal correction to $\mathrm{H}=0.156084$

Thermal correction to $\mathrm{G}=0.099172$

$\mathrm{E}(\operatorname{CASPT} 2(6,6) / 6-31 \mathrm{G}(\mathrm{d}))=-271.6722149799$

\section{$(6,6) \operatorname{CASSCF} / 6-31+\mathrm{G} *$}

$\begin{array}{lrrr}\mathrm{C} & 2.62243 & 0.78397 & -0.09245 \\ \mathrm{C} & 2.08803 & -0.47073 & -0.37518 \\ \mathrm{C} & 0.85413 & -0.97752 & 0.03037 \\ \mathrm{C} & -0.17531 & -0.25918 & 0.86697 \\ \mathrm{C} & -1.42028 & 0.14902 & 0.08462 \\ \mathrm{C} & -2.31167 & -0.84341 & -0.33695 \\ \mathrm{C} & -1.64762 & 1.49086 & -0.19831 \\ \mathrm{H} & -0.97161 & 2.25906 & 0.12791 \\ \mathrm{H} & -2.50870 & 1.80057 & -0.76239 \\ \mathrm{H} & -0.47687 & -0.91608 & 1.67958 \\ \mathrm{H} & 0.25750 & 0.61702 & 1.33236 \\ \mathrm{H} & 2.69447 & -1.12746 & -0.97802 \\ \mathrm{H} & 2.10418 & 1.51564 & 0.49735 \\ \mathrm{H} & 0.59452 & -1.96876 & -0.29896 \\ \mathrm{H} & -2.14872 & -1.88099 & -0.11032 \\ \mathrm{H} & -3.19441 & -0.59533 & -0.89720 \\ \mathrm{H} & 3.59137 & 1.05834 & -0.46461\end{array}$

$\mathrm{E}(\operatorname{CASSCF}(6,6) / 6-31+\mathrm{G}(\mathrm{d}))=-270.8512812727$

$\mathrm{E}(\operatorname{CASPT} 2(6,6) / 6-31 \mathrm{G}(\mathrm{d}))=-271.6713329962$
TS-5-5

$\mathrm{UB} 3 \mathrm{LYP} / 6-31+\mathrm{G}(\mathrm{d})$ :

$\begin{array}{lrrr}\mathrm{C} & -2.55505 & -0.80423 & -0.62672 \\ \mathrm{C} & -2.17578 & 0.38813 & -0.02875 \\ \mathrm{C} & -0.96614 & 0.67245 & 0.60721 \\ \mathrm{C} & 0.16967 & -0.28591 & 0.80965 \\ \mathrm{C} & 1.44598 & -0.01355 & -0.00413 \\ \mathrm{C} & 1.67736 & 1.22271 & -0.59164 \\ \mathrm{C} & 2.37382 & -1.04978 & -0.11144 \\ \mathrm{H} & 2.20356 & -2.01867 & 0.34987 \\ \mathrm{H} & 3.29862 & -0.91837 & -0.66640 \\ \mathrm{H} & 0.45344 & -0.27181 & 1.87416 \\ \mathrm{H} & -0.15592 & -1.31101 & 0.60400 \\ \mathrm{H} & -2.90103 & 1.20222 & -0.05914 \\ \mathrm{H} & -1.90467 & -1.67300 & -0.66194 \\ \mathrm{H} & -0.83840 & 1.67520 & 1.01107 \\ \mathrm{H} & 0.96003 & 2.03369 & -0.53788 \\ \mathrm{H} & 2.59454 & 1.40681 & -1.14446 \\ \mathrm{H} & -3.52940 & -0.90400 & -1.09439\end{array}$

$\mathrm{E}(\mathrm{UB}+\mathrm{HF}-\mathrm{LYP})=-272.650459976$

$S * * 2=1.0487$

1 imaginary frequency

$383 \mathrm{~K}, 1.00 \mathrm{~atm}$, freq's scaled by 1.0000

Zero-point correction $=0.142904$

Thermal correction to $\mathrm{U}=0.154811$

Thermal correction to $\mathrm{H}=0.156024$

Thermal correction to $\mathrm{G}=0.098174$

$\mathrm{E}(\operatorname{CASPT} 2(6,6) / 6-31 \mathrm{G}(\mathrm{d}))=-271.6713031821$

$(6,6) \mathrm{CASSCF} / 6-31+\mathrm{G}^{*}$ :

$\begin{array}{lrrr}\mathrm{C} & -2.36797 & -0.77056 & -0.81422 \\ \mathrm{C} & -2.14592 & 0.30684 & 0.03674 \\ \mathrm{C} & -1.00486 & 0.54767 & 0.80507 \\ \mathrm{C} & 0.20584 & -0.34287 & 0.88348 \\ \mathrm{C} & 1.38627 & 0.03028 & -0.01702 \\ \mathrm{C} & 1.44765 & 1.24485 & -0.68627 \\ \mathrm{C} & 2.43025 & -0.89952 & -0.12208 \\ \mathrm{H} & 2.39789 & -1.84162 & 0.39413 \\ \mathrm{H} & 3.29725 & -0.69570 & -0.72311 \\ \mathrm{H} & 0.56114 & -0.34428 & 1.91128 \\ \mathrm{H} & -0.07573 & -1.36754 & 0.66792 \\ \mathrm{H} & -2.93471 & 1.03822 & 0.11091 \\ \mathrm{H} & -1.63795 & -1.54217 & -0.96983 \\ \mathrm{H} & -0.98156 & 1.45325 & 1.38635 \\ \mathrm{H} & 0.65805 & 1.96850 & -0.63684 \\ \mathrm{H} & 2.29820 & 1.48666 & -1.29755 \\ \mathrm{H} & -3.29016 & -0.85554 & -1.35746\end{array}$

$\mathrm{E}(\operatorname{CASSCF}(6,6) / 6-31+\mathrm{G}(\mathrm{d}))=-270.850399451$

$\mathrm{E}(\operatorname{CASPT} 2(6,6) / 6-31 \mathrm{G}(\mathrm{d}))=-271.670842142$ 
UB3LYP / 6-31+G (d) :

$\begin{array}{lrrr}\mathrm{C} & -2.97570 & 0.34798 & -0.51082 \\ \mathrm{C} & -2.20363 & 0.25702 & 0.63404 \\ \mathrm{C} & -0.81756 & 0.08221 & 0.69169 \\ \mathrm{C} & 0.08413 & -0.04807 & -0.50719 \\ \mathrm{C} & 1.54557 & 0.20557 & -0.16392 \\ \mathrm{C} & 2.40010 & -0.87225 & 0.02743 \\ \mathrm{C} & 1.97256 & 1.52452 & -0.01812 \\ \mathrm{H} & 1.29399 & 2.35931 & -0.16671 \\ \mathrm{H} & 3.00065 & 1.75742 & 0.24585 \\ \mathrm{H} & -0.01741 & -1.05346 & -0.94668 \\ \mathrm{H} & -0.23413 & 0.65178 & -1.29279 \\ \mathrm{H} & -2.72154 & 0.32458 & 1.59126 \\ \mathrm{H} & -2.54497 & 0.28549 & -1.50616 \\ \mathrm{H} & -0.34846 & 0.00284 & 1.66983 \\ \mathrm{H} & 2.05953 & -1.89652 & -0.09254 \\ \mathrm{H} & 3.44039 & -0.72304 & 0.30431 \\ \mathrm{H} & -4.05067 & 0.48603 & -0.45028\end{array}$

$\mathrm{E}(\mathrm{UB}+\mathrm{HF}-\mathrm{LYP})=-272.653408276$

$\mathrm{S} * * 2=1.0221$

$383 \mathrm{~K}, 1.00 \mathrm{~atm}$, freq's scaled by 1.0000

Zero-point correction $=0.142907$

Thermal correction to $U=0.155962$

Thermal correction to $\mathrm{H}=0.157175$

Thermal correction to $\mathrm{G}=0.095893$

$\mathrm{E}(\operatorname{CASPT} 2(6,6) / 6-31 \mathrm{G}(\mathrm{d}))=-271.6744334955$

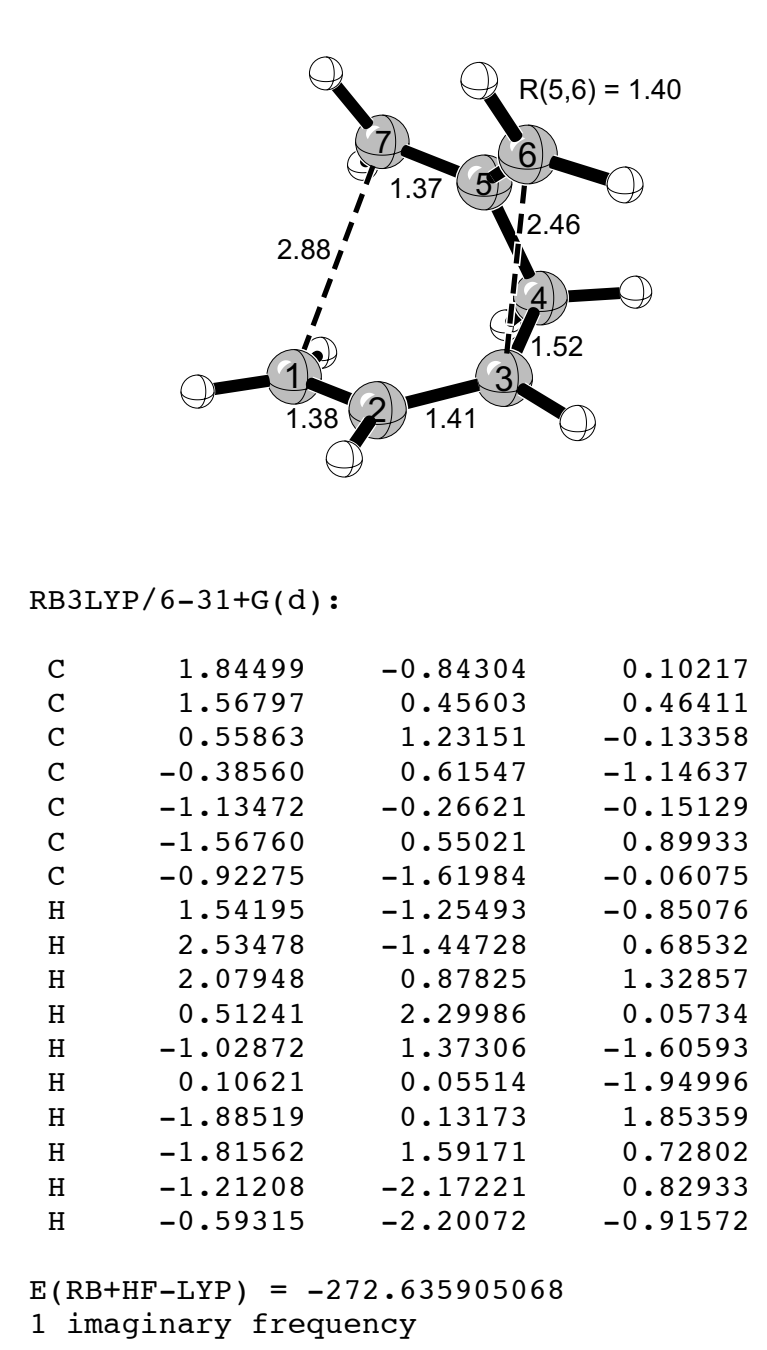




\section{TS-1c-5c}

UB3LYP / 6-31+G (d) :

$\begin{array}{lrrr}\mathrm{C} & 1.84001 & 2.87226 & -0.00452 \\ \mathrm{C} & 0.81198 & 1.98417 & -0.01382 \\ \mathrm{C} & 0.86341 & 0.60139 & -0.40846 \\ \mathrm{C} & 2.07943 & -0.07886 & -1.01246 \\ \mathrm{C} & 2.29711 & -1.14793 & 0.03826 \\ \mathrm{C} & 2.07237 & -0.51316 & 1.30858 \\ \mathrm{C} & 2.37272 & -2.47844 & -0.20344 \\ \mathrm{H} & 2.42060 & -2.87175 & -1.21510 \\ \mathrm{H} & 2.37665 & -3.19933 & 0.61094 \\ \mathrm{H} & 1.87202 & -0.46668 & -2.01574 \\ \mathrm{H} & 2.92739 & 0.61062 & -1.07943 \\ \mathrm{H} & -0.16136 & 2.33261 & 0.32858 \\ \mathrm{H} & 2.84132 & 2.62082 & -0.34091 \\ \mathrm{H} & 1.85410 & -1.11046 & 2.19435 \\ \mathrm{H} & 2.47275 & 0.47698 & 1.49921 \\ \mathrm{H} & 1.68693 & 3.88814 & 0.34626 \\ \mathrm{O} & -0.28874 & -0.04435 & -0.70604 \\ \mathrm{Si} & -1.82111 & -0.17715 & 0.02143 \\ \mathrm{C} & -1.63186 & -0.36778 & 1.88460 \\ \mathrm{H} & -1.15695 & 0.50667 & 2.34434 \\ \mathrm{H} & -2.61714 & -0.49677 & 2.35199 \\ \mathrm{H} & -1.02378 & -1.24479 & 2.13396 \\ \mathrm{C} & -2.86802 & 1.33593 & -0.40126 \\ \mathrm{H} & -2.84822 & 1.53948 & -1.47905 \\ \mathrm{H} & -3.91470 & 1.16459 & -0.11598 \\ \mathrm{H} & -2.53284 & 2.24237 & 0.11668 \\ \mathrm{C} & -2.55070 & -1.71520 & -0.77252 \\ \mathrm{H} & -1.93576 & -2.59726 & -0.55917 \\ \mathrm{H} & -3.56479 & -1.90988 & -0.40034 \\ \mathrm{H} & -2.60943 & -1.60477 & -1.86217\end{array}$

$\mathrm{E}(\mathrm{UB}+\mathrm{HF}-\mathrm{LYP})=-756.581127274$

$\mathrm{S} * * 2=0.3606$

1 imaginary frequency

$383 \mathrm{~K}, 1.00 \mathrm{~atm}$, freq's scaled by 1.0000

Zero-point correction $=0.250478$

Thermal correction to $U=0.275607$

Thermal correction to $\mathrm{H}=0.276820$

Thermal correction to $\mathrm{G}=0.188688$

$\mathrm{E}(\operatorname{CASPT} 2(6,6) / 6-31 \mathrm{G}(\mathrm{d}))=-754.4521349939$

\section{TS-1c-6c}

UB3LYP/ $6-31+G(d)$ :

$\begin{array}{lrrr}\mathrm{C} & 1.92832 & 2.25815 & 0.19409 \\ \mathrm{C} & 0.85724 & 1.42728 & 0.29946 \\ \mathrm{C} & 0.70806 & 0.16936 & -0.37476 \\ \mathrm{C} & 1.82214 & -0.43045 & -1.20805 \\ \mathrm{C} & 1.92934 & -1.45628 & 0.91557 \\ \mathrm{C} & 2.75090 & -0.87772 & -0.09509 \\ \mathrm{C} & 4.04066 & -0.47213 & 0.03930 \\ \mathrm{H} & 1.06628 & -2.05251 & 0.63698 \\ \mathrm{H} & 2.29903 & -1.59275 & 1.93123 \\ \mathrm{H} & 1.41780 & -1.27668 & -1.77428 \\ \mathrm{H} & 2.27147 & 0.27067 & -1.92299 \\ \mathrm{H} & 0.04713 & 1.71590 & 0.96675 \\ \mathrm{H} & 2.75627 & 2.06403 & -0.47717 \\ \mathrm{H} & 4.55425 & 0.06731 & -0.75180 \\ \mathrm{H} & 4.60798 & -0.68708 & 0.94137 \\ \mathrm{H} & 1.98737 & 3.15982 & 0.79682 \\ \mathrm{O} & -0.51648 & -0.35622 & -0.60712 \\ \mathrm{Si} & -2.08295 & -0.09559 & 0.01774 \\ \mathrm{C} & -2.70394 & 1.61206 & -0.48422 \\ \mathrm{H} & -3.75604 & 1.73304 & -0.19333 \\ \mathrm{H} & -2.13580 & 2.42416 & -0.01703 \\ \mathrm{H} & -2.64338 & 1.74610 & -1.57125 \\ \mathrm{C} & -2.07525 & -0.30940 & 1.89015 \\ \mathrm{H} & -3.10159 & -0.26188 & 2.27789 \\ \mathrm{H} & -1.65739 & -1.28238 & 2.17546 \\ \mathrm{H} & -1.49335 & 0.46490 & 2.40313 \\ \mathrm{C} & -3.09267 & -1.44252 & -0.81589 \\ \mathrm{H} & -4.14869 & -1.38302 & -0.52269 \\ \mathrm{H} & -3.04228 & -1.35224 & -1.90766 \\ \mathrm{H} & -2.72475 & -2.43964 & -0.54613\end{array}$

$\mathrm{E}(\mathrm{UB}+\mathrm{HF}-\mathrm{LYP})=-756.583309157$

$\mathrm{S} * * 2=0.2917$

1 imaginary frequency

$383 \mathrm{~K}, 1.00 \mathrm{~atm}$, freq's scaled by 1.0000

Zero-point correction $=0.250440$

Thermal correction to $\mathrm{U}=0.275615$

Thermal correction to $\mathrm{H}=0.276828$

Thermal correction to $\mathrm{G}=0.188524$

$\mathrm{E}(\operatorname{CASPT} 2(6,6) / 6-31 \mathrm{G}(\mathrm{d}))=-754.454484434$ 
$5 c$

UB3LYP / 6-31+G (d) :

$\begin{array}{lrrr}\mathrm{C} & 3.39776 & 0.89227 & 0.00000 \\ \mathrm{C} & 2.22673 & 0.15287 & 0.00000 \\ \mathrm{C} & 0.92593 & 0.66932 & 0.00000 \\ \mathrm{C} & 0.65827 & 2.16121 & 0.00000 \\ \mathrm{C} & -0.80796 & 2.56870 & 0.00000 \\ \mathrm{C} & -1.45744 & 2.76056 & 1.21520 \\ \mathrm{C} & -1.45744 & 2.76056 & -1.21520 \\ \mathrm{H} & -0.94615 & 2.61749 & -2.16260 \\ \mathrm{H} & -2.50339 & 3.05317 & -1.25204 \\ \mathrm{H} & 1.15667 & 2.60532 & 0.87433 \\ \mathrm{H} & 1.15667 & 2.60532 & -0.87433 \\ \mathrm{H} & 2.32516 & -0.93142 & 0.00000 \\ \mathrm{H} & 3.40410 & 1.97771 & 0.00000 \\ \mathrm{H} & -0.94615 & 2.61749 & 2.16260 \\ \mathrm{H} & -2.50339 & 3.05317 & 1.25204 \\ \mathrm{H} & 4.36258 & 0.39613 & 0.00000 \\ \mathrm{O} & -0.17533 & -0.11961 & 0.00000 \\ \mathrm{Si} & -0.47302 & -1.78952 & 0.00000 \\ \mathrm{C} & -2.34909 & -1.88609 & 0.00000 \\ \mathrm{H} & -2.76730 & -1.39204 & -0.88504 \\ \mathrm{H} & -2.69368 & -2.92823 & 0.00000 \\ \mathrm{H} & -2.76730 & -1.39204 & 0.88504 \\ \mathrm{C} & 0.24085 & -2.57950 & -1.55789 \\ \mathrm{H} & 1.33556 & -2.53741 & -1.59082 \\ \mathrm{H} & -0.05122 & -3.63653 & -1.61656 \\ \mathrm{H} & -0.13835 & -2.08078 & -2.45838 \\ \mathrm{C} & 0.24085 & -2.57950 & 1.55789 \\ \mathrm{H} & -0.13835 & -2.08078 & 2.45838 \\ \mathrm{H} & -0.05122 & -3.63653 & 1.61656 \\ \mathrm{H} & 1.33556 & -2.53741 & 1.59082 \\ & & & \end{array}$

$\mathrm{E}(\mathrm{UB}+\mathrm{HF}-\mathrm{LYP})=-756.594416108$ $\mathrm{S} * * 2=1.0468$

1 imaginary frequency

$383 \mathrm{~K}, 1.00 \mathrm{~atm}$, freq's scaled by 1.0000

zero-point correction $=0.249045$

Thermal correction to $\mathrm{U}=0.274546$

Thermal correction to $\mathrm{H}=0.275759$

Thermal correction to $\mathrm{G}=0.186131$

$\mathrm{E}(\operatorname{CASPT} 2(6,6) / 6-31 \mathrm{G}(\mathrm{d}))=-754.4572708402$ 6c

UB3LYP/ $6-31+G(d):$

$\begin{array}{lrrr}\mathrm{C} & 1.42884 & 3.01767 & 0.01796 \\ \mathrm{C} & 0.47822 & 2.01471 & -0.09509 \\ \mathrm{C} & 0.67545 & 0.69637 & -0.51820 \\ \mathrm{C} & 1.98660 & 0.11684 & -0.96890 \\ \mathrm{C} & 2.57054 & -0.90449 & 0.01321 \\ \mathrm{C} & 2.81154 & -2.20161 & -0.41312 \\ \mathrm{C} & 2.84756 & -0.48963 & 1.31676 \\ \mathrm{O} & -0.34253 & -0.20152 & -0.59544 \\ \mathrm{Si} & -1.90030 & -0.31734 & 0.07083 \\ \mathrm{C} & -2.41104 & -2.08053 & -0.33099 \\ \mathrm{C} & -1.83086 & -0.03350 & 1.93341 \\ \mathrm{C} & -3.06332 & 0.90968 & -0.76844 \\ \mathrm{H} & -2.81185 & 1.95383 & -0.54885 \\ \mathrm{H} & 2.63596 & 0.52183 & 1.64918 \\ \mathrm{H} & 3.28552 & -1.17374 & 2.03871 \\ \mathrm{H} & 1.83714 & -0.36678 & -1.94284 \\ \mathrm{H} & 2.70951 & 0.92417 & -1.12309 \\ \mathrm{H} & -0.54295 & 2.28182 & 0.17341 \\ \mathrm{H} & 2.47612 & 2.86865 & -0.22517 \\ \mathrm{H} & 2.58950 & -2.51875 & -1.42752 \\ \mathrm{H} & 3.22260 & -2.94661 & 0.26335 \\ \mathrm{H} & 1.14136 & 4.00737 & 0.35672 \\ \mathrm{H} & -1.72374 & -2.80313 & 0.12472 \\ \mathrm{H} & -3.42182 & -2.29469 & 0.03964 \\ \mathrm{H} & -2.40737 & -2.25377 & -1.41396 \\ \mathrm{H} & -1.54726 & 0.99266 & 2.19393 \\ \mathrm{H} & -2.81323 & -0.22989 & 2.38313 \\ \mathrm{H} & -1.10702 & -0.70916 & 2.40500 \\ \mathrm{H} & -3.04245 & 0.78470 & -1.85823 \\ \mathrm{H} & -4.09675 & 0.74327 & -0.43590\end{array}$

$\mathrm{E}(\mathrm{UB}+\mathrm{HF}-\mathrm{LYP})=-756.598003493$

$\mathrm{S} * * 2=1.0273$

$383 \mathrm{~K}, 1.00 \mathrm{~atm}$, freq's scaled by 1.0000

Zero-point correction $=0.249542$

Thermal correction to $\mathrm{U}=0.276047$

Thermal correction to $\mathrm{H}=0.277260$

Thermal correction to $\mathrm{G}=0.184024$

$\mathrm{E}(\operatorname{CASPT} 2(6,6) / 6-31 \mathrm{G}(\mathrm{d}))=-754.4615878711$ 
TS $-6 c-2 c-x$

UB3LYP / 6-31+G (d) :

$\begin{array}{lrrr}\mathrm{C} & 1.99347 & 2.09661 & 0.21774 \\ \mathrm{C} & 0.82676 & 1.33404 & 0.34001 \\ \mathrm{C} & 0.60242 & 0.17799 & -0.39350 \\ \mathrm{C} & 1.72472 & -0.50345 & -1.13327 \\ \mathrm{C} & 2.83300 & -0.80727 & -0.11594 \\ \mathrm{C} & 2.64203 & -1.87992 & 0.72108 \\ \mathrm{C} & 3.87901 & 0.11797 & 0.03033 \\ \mathrm{O} & -0.57297 & -0.49687 & -0.41380 \\ \mathrm{Si} & -2.16347 & -0.08450 & 0.03670 \\ \mathrm{C} & -2.31660 & -0.01382 & 1.91539 \\ \mathrm{C} & -3.17776 & -1.50747 & -0.65427 \\ \mathrm{C} & -2.64467 & 1.55530 & -0.75705 \\ \mathrm{H} & -2.02930 & 2.38778 & -0.39802 \\ \mathrm{H} & 1.76875 & -2.52002 & 0.63337 \\ \mathrm{H} & 3.37154 & -2.13696 & 1.48494 \\ \mathrm{H} & 1.33806 & -1.42021 & -1.58680 \\ \mathrm{H} & 2.12019 & 0.12531 & -1.94399 \\ \mathrm{H} & 0.10687 & 1.60736 & 1.11021 \\ \mathrm{H} & 2.54962 & 2.13868 & -0.70734 \\ \mathrm{H} & 4.18397 & 0.75981 & -0.78831 \\ \mathrm{H} & 4.56036 & 0.04806 & 0.87416 \\ \mathrm{H} & 2.20906 & 2.88069 & 0.93793 \\ \mathrm{H} & -1.78713 & 0.84037 & 2.35299 \\ \mathrm{H} & -3.37220 & 0.06987 & 2.20691 \\ \mathrm{H} & -1.91682 & -0.92548 & 2.37620 \\ \mathrm{H} & -4.24471 & -1.37890 & -0.43119 \\ \mathrm{H} & -3.06796 & -1.57811 & -1.74308 \\ \mathrm{H} & -2.85847 & -2.46445 & -0.22407 \\ \mathrm{H} & -2.54119 & 1.51000 & -1.84818 \\ \mathrm{H} & -3.69256 & 1.79429 & -0.53222\end{array}$

$\mathrm{E}(\mathrm{UB}+\mathrm{HF}-\mathrm{LYP})=-756.589783399$

$\mathrm{S} * * 2=0.5455$

1 imaginary frequency

$383 \mathrm{~K}, 1.00 \mathrm{~atm}$, freq's scaled by 1.0000

Zero-point correction $=0.250487$

Thermal correction to $U=0.275384$

Thermal correction to $\mathrm{H}=0.276597$

Thermal correction to $\mathrm{G}=0.188901$

$\mathrm{E}(\operatorname{CASPT} 2(6,6) / 6-31 \mathrm{G}(\mathrm{d}))=-754.4596184345$

\section{TS $-6 c-2 c-n$}

$\mathrm{UB} 3 \mathrm{LYP} / 6-31+\mathrm{G}(\mathrm{d})$ :

$\begin{array}{lrrr}\mathrm{C} & -1.87623 & 1.99858 & 0.59151 \\ \mathrm{C} & -0.68151 & 1.34696 & 0.24413 \\ \mathrm{C} & -0.54332 & -0.02576 & 0.15928 \\ \mathrm{C} & -1.67684 & -0.99900 & 0.33533 \\ \mathrm{C} & -3.05561 & -0.58295 & -0.19793 \\ \mathrm{C} & -4.15060 & -1.24866 & 0.30160 \\ \mathrm{C} & -3.18262 & 0.45332 & -1.14527 \\ \mathrm{O} & 0.64001 & -0.64147 & -0.10824 \\ \mathrm{Si} & 2.26559 & -0.14489 & -0.04513 \\ \mathrm{C} & 2.65250 & 1.03374 & -1.46731 \\ \mathrm{C} & 3.21009 & -1.75586 & -0.25621 \\ \mathrm{C} & 2.62328 & 0.65437 & 1.62398 \\ \mathrm{H} & 3.68364 & 0.92975 & 1.69728 \\ \mathrm{H} & -2.34108 & 0.83028 & -1.70878 \\ \mathrm{H} & -4.17448 & 0.75251 & -1.47525 \\ \mathrm{H} & -1.36927 & -1.93588 & -0.14548 \\ \mathrm{H} & -1.79352 & -1.23827 & 1.40554 \\ \mathrm{H} & 0.15815 & 1.96316 & -0.07359 \\ \mathrm{H} & -2.65254 & 1.52528 & 1.17635 \\ \mathrm{H} & -4.05197 & -2.01990 & 1.06119 \\ \mathrm{H} & -5.15179 & -1.04464 & -0.06849 \\ \mathrm{H} & -1.94981 & 3.07690 & 0.47985 \\ \mathrm{H} & 2.17177 & 2.01212 & -1.35244 \\ \mathrm{H} & 3.73520 & 1.20617 & -1.53260 \\ \mathrm{H} & 2.32664 & 0.61172 & -2.42607 \\ \mathrm{H} & 2.95213 & -2.24104 & -1.20534 \\ \mathrm{H} & 4.29411 & -1.58397 & -0.25093 \\ \mathrm{H} & 2.97668 & -2.45909 & 0.55215 \\ \mathrm{H} & 2.03146 & 1.56286 & 1.78285 \\ \mathrm{H} & 2.40144 & -0.03617 & 2.44693\end{array}$

$\mathrm{E}(\mathrm{UB}+\mathrm{HF}-\mathrm{LYP})=-756.589870495$

$\mathrm{S} * * 2=0.6594$

1 imaginary frequency

$383 \mathrm{~K}, 1.00 \mathrm{~atm}$, freq's scaled by 1.0000

Zero-point correction $=0.250089$

Thermal correction to $\mathrm{U}=0.275135$

Thermal correction to $\mathrm{H}=0.276348$

Thermal correction to $\mathrm{G}=0.188089$

$\mathrm{E}(\operatorname{CASPT} 2(6,6) / 6-31 \mathrm{G}(\mathrm{d}))=-754.4581956393$ 
TS-6c-6c

UB3LYP / 6-31+G (d) :

$\begin{array}{lrrr}\mathrm{C} & 1.43262 & 2.84667 & 0.28992 \\ \mathrm{C} & 0.46132 & 1.86823 & 0.15347 \\ \mathrm{C} & 0.63316 & 0.58023 & -0.36381 \\ \mathrm{C} & 1.91997 & 0.04616 & -0.92014 \\ \mathrm{C} & 2.80144 & -0.78298 & 0.02886 \\ \mathrm{C} & 2.37347 & -1.12292 & 1.30304 \\ \mathrm{C} & 4.05012 & -1.18418 & -0.44907 \\ \mathrm{O} & -0.38299 & -0.32584 & -0.41835 \\ \mathrm{Si} & -2.03352 & -0.32267 & -0.02034 \\ \mathrm{H} & 4.38906 & -0.92459 & -1.44836 \\ \mathrm{H} & 4.72464 & -1.77057 & 0.16860 \\ \mathrm{H} & 1.67176 & -0.58281 & -1.78595 \\ \mathrm{H} & 2.51918 & 0.87930 & -1.30497 \\ \mathrm{H} & -0.54291 & 2.12619 & 0.48687 \\ \mathrm{H} & 2.46958 & 2.68518 & 0.01304 \\ \mathrm{H} & 1.40937 & -0.81245 & 1.68924 \\ \mathrm{H} & 3.00794 & -1.71402 & 1.95805 \\ \mathrm{H} & 1.17832 & 3.81927 & 0.69777 \\ \mathrm{C} & -2.26240 & -0.04262 & 1.83198 \\ \mathrm{H} & -1.92201 & 0.94732 & 2.15681 \\ \mathrm{H} & -3.32348 & -0.13154 & 2.10077 \\ \mathrm{H} & -1.71094 & -0.79199 & 2.41327 \\ \mathrm{C} & -2.59259 & -2.04958 & -0.50594 \\ \mathrm{H} & -3.65901 & -2.19659 & -0.29152 \\ \mathrm{H} & -2.43717 & -2.22779 & -1.57679 \\ \mathrm{H} & -2.03022 & -2.81380 & 0.04380 \\ \mathrm{C} & -2.94569 & 0.98596 & -1.02794 \\ \mathrm{H} & -2.74730 & 0.86294 & -2.09985 \\ \mathrm{H} & -4.02964 & 0.89190 & -0.87874 \\ \mathrm{H} & -2.66257 & 2.00836 & -0.75269\end{array}$

$\mathrm{E}(\mathrm{UB}+\mathrm{HF}-\mathrm{LYP})=-756.595814001$

$\mathrm{S} * * 2=1.0507$

1 imaginary frequency

$383 \mathrm{~K}, 1.00 \mathrm{~atm}$, freq's scaled by 1.0000

Zero-point correction $=0.249198$

Thermal correction to $\mathrm{U}=0.274643$

Thermal correction to $\mathrm{H}=0.275855$

Thermal correction to $\mathrm{G}=0.186072$

$\mathrm{E}(\operatorname{CASPT} 2(6,6) / 6-31 \mathrm{G}(\mathrm{d}))=-754.4590578062$ 


\section{TS-1d-5Zd-cis}

UB3LYP / 6-31+G (d) :

$\begin{array}{lrrr}\text { C } & -4.38334 & -0.90066 & -0.39664 \\ \mathrm{C} & -3.70258 & -0.40321 & 0.67705 \\ \mathrm{C} & -2.29216 & -0.24151 & 0.78211 \\ \mathrm{C} & -1.26678 & -0.64552 & -0.24493 \\ \mathrm{C} & -0.47826 & 0.65343 & -0.32804 \\ \mathrm{C} & 0.88448 & 0.80541 & -0.14953 \\ \mathrm{C} & -1.43822 & 1.70446 & -0.33235 \\ \mathrm{C} & 1.76420 & -0.37722 & -0.13738 \\ \mathrm{C} & 1.54159 & 2.15449 & 0.03991 \\ \mathrm{H} & -0.66268 & -1.50618 & 0.05078 \\ \mathrm{H} & -1.72466 & -0.88966 & -1.21065 \\ \mathrm{H} & -4.28348 & -0.08560 & 1.54313 \\ \mathrm{H} & -3.88281 & -1.24535 & -1.29700 \\ \mathrm{H} & -1.89216 & 0.01218 & 1.76047 \\ \mathrm{H} & -1.19339 & 2.73627 & -0.09568 \\ \mathrm{H} & -2.39025 & 1.55173 & -0.82675 \\ \mathrm{H} & -5.46591 & -0.98288 & -0.38043 \\ \mathrm{O} & 3.03609 & -0.05095 & 0.23623 \\ \mathrm{O} & 1.46407 & -1.53017 & -0.42727 \\ \mathrm{C} & 3.97926 & -1.13221 & 0.24796 \\ \mathrm{H} & 4.92938 & -0.68363 & 0.54212 \\ \mathrm{H} & 3.67748 & -1.89919 & 0.96710 \\ \mathrm{H} & 4.05754 & -1.58581 & -0.74433 \\ \mathrm{H} & 2.36354 & 2.29698 & -0.67126 \\ \mathrm{H} & 0.83757 & 2.97715 & -0.10004 \\ \mathrm{H} & 1.97938 & 2.24824 & 1.04194\end{array}$

$\mathrm{E}(\mathrm{UB}+\mathrm{HF}-\mathrm{LYP})=-539.848669759$

$\mathrm{S} * * 2=0.4499$

1 imaginary frequency

$383 \mathrm{~K}, 1.00 \mathrm{~atm}$, freq's scaled by 1.0000

Zero-point correction $=0.216037$

Thermal correction to $U=0.236585$

Thermal correction to $\mathrm{H}=0.237798$

Thermal correction to $\mathrm{G}=0.159365$

\section{TS-1d-5Zd-trans}

UB3LYP/6-31+G (d) :

$\begin{array}{rrrr}\mathrm{C} & -4.16658 & -1.01188 & -0.41589 \\ \mathrm{C} & -3.56132 & -0.37395 & 0.62825 \\ \mathrm{C} & -2.16535 & -0.14199 & 0.78452 \\ \mathrm{C} & -1.07153 & -0.61552 & -0.14058 \\ \mathrm{C} & -0.33493 & 0.70209 & -0.33175 \\ \mathrm{C} & 0.99569 & 0.99354 & -0.09995 \\ \mathrm{C} & -1.33870 & 1.69848 & -0.49356 \\ \mathrm{C} & 2.05512 & -0.01952 & 0.07239 \\ \mathrm{C} & 1.47054 & 2.42990 & -0.01611 \\ \mathrm{H} & -0.45948 & -1.41010 & 0.28875 \\ \mathrm{H} & -1.47039 & -0.98784 & -1.09150 \\ \mathrm{H} & -4.19834 & 0.01197 & 1.42425 \\ \mathrm{H} & -3.60826 & -1.43029 & -1.24857 \\ \mathrm{H} & -1.82619 & 0.22841 & 1.74842 \\ \mathrm{H} & -1.13591 & 2.75752 & -0.35541 \\ \mathrm{H} & -2.25757 & 1.45817 & -1.01541 \\ \mathrm{H} & -5.24500 & -1.13494 & -0.44370 \\ \mathrm{O} & 1.68507 & -1.29255 & -0.25317 \\ \mathrm{O} & 3.19361 & 0.23187 & 0.44511 \\ \mathrm{C} & 2.70653 & -2.29132 & -0.09839 \\ \mathrm{H} & 2.23772 & -3.23116 & -0.39449 \\ \mathrm{H} & 3.56069 & -2.06780 & -0.74350 \\ \mathrm{H} & 3.04688 & -2.33946 & 0.93982 \\ \mathrm{H} & 2.53864 & 2.45832 & 0.20466 \\ \mathrm{H} & 1.30168 & 2.96854 & -0.95778 \\ \mathrm{H} & 0.94920 & 2.98507 & 0.77541 \\ \mathrm{H} & & & \end{array}$

$\mathrm{E}(\mathrm{UB}+\mathrm{HF}-\mathrm{LYP})=-539.846411832$

$\mathrm{S} * * 2=0.4496$

1 imaginary frequency

$383 \mathrm{~K}, 1.00 \mathrm{~atm}$, freq's scaled by 1.0000

Zero-point correction $=0.216000$

Thermal correction to $\mathrm{U}=0.236592$

Thermal correction to $\mathrm{H}=0.237805$

Thermal correction to $\mathrm{G}=0.159112$ 


\section{TS-1d-6Zd-cis}

UB3LYP / 6-31+G (d) :

$\begin{array}{lrrr}\mathrm{C} & 1.41151 & -1.81132 & 1.32361 \\ \mathrm{C} & 2.54958 & -1.20071 & 0.88055 \\ \mathrm{C} & 2.67740 & -0.50765 & -0.35452 \\ \mathrm{C} & 1.51125 & -0.29052 & -1.28932 \\ \mathrm{C} & 0.88538 & 0.89031 & -0.54623 \\ \mathrm{C} & -0.36799 & 0.91027 & 0.04849 \\ \mathrm{C} & 1.93913 & 1.78909 & -0.26677 \\ \mathrm{H} & 2.76928 & 1.86932 & -0.95897 \\ \mathrm{H} & 1.86597 & 2.59033 & 0.46284 \\ \mathrm{H} & 1.85044 & -0.00231 & -2.29004 \\ \mathrm{H} & 0.84441 & -1.14532 & -1.39302 \\ \mathrm{H} & 3.43307 & -1.22568 & 1.51804 \\ \mathrm{H} & 0.51306 & -1.87453 & 0.71949 \\ \mathrm{H} & 3.68126 & -0.27639 & -0.69995 \\ \mathrm{C} & -1.39511 & -0.06549 & -0.34829 \\ \mathrm{C} & -0.77022 & 1.95332 & 1.06939 \\ \mathrm{H} & 1.38405 & -2.29514 & 2.29582 \\ \mathrm{H} & -1.16215 & 1.48527 & 1.97877 \\ \mathrm{H} & 0.06648 & 2.59243 & 1.35754 \\ \mathrm{H} & -1.56738 & 2.60233 & 0.68420 \\ \mathrm{O} & -2.55187 & 0.10923 & 0.35754 \\ \mathrm{O} & -1.30458 & -0.94585 & -1.19975 \\ \mathrm{C} & -3.62791 & -0.77716 & 0.02020 \\ \mathrm{H} & -4.46088 & -0.47532 & 0.65715 \\ \mathrm{H} & -3.89411 & -0.67650 & -1.03596 \\ \mathrm{H} & -3.35004 & -1.81638 & 0.21902\end{array}$

$\mathrm{E}(\mathrm{UB}+\mathrm{HF}-\mathrm{LYP})=-539.847119151$

$\mathrm{S} * * 2=0.3024$

1 imaginary frequency

$383 \mathrm{~K}, 1.00 \mathrm{~atm}$, freq's scaled by 1.0000

Zero-point correction $=0.216741$

Thermal correction to $U=0.237036$

Thermal correction to $\mathrm{H}=0.238249$

Thermal correction to $\mathrm{G}=0.160448$

\section{TS-1d-6Zd-trans}

UB3LYP/ $6-31+G(d)$ :

$\begin{array}{lrrr}\mathrm{C} & 1.44334 & -1.35797 & 1.77581 \\ \mathrm{C} & 2.50429 & -1.07257 & 0.96571 \\ \mathrm{C} & 2.42819 & -0.81981 & -0.43200 \\ \mathrm{C} & 1.11585 & -0.76136 & -1.18046 \\ \mathrm{C} & 0.72855 & 0.66347 & -0.78341 \\ \mathrm{C} & -0.35453 & 1.07020 & -0.02074 \\ \mathrm{C} & 1.88133 & 1.45922 & -0.97621 \\ \mathrm{H} & 2.57005 & 1.22708 & -1.78056 \\ \mathrm{H} & 1.99488 & 2.44301 & -0.52926 \\ \mathrm{H} & 1.26876 & -0.85417 & -2.26117 \\ \mathrm{H} & 0.39597 & -1.51944 & -0.87741 \\ \mathrm{H} & 3.49512 & -1.01202 & 1.41521 \\ \mathrm{H} & 0.43544 & -1.47924 & 1.39447 \\ \mathrm{H} & 3.35548 & -0.83758 & -0.99762 \\ \mathrm{C} & -1.60168 & 0.30144 & 0.13937 \\ \mathrm{C} & -0.35570 & 2.42577 & 0.65769 \\ \mathrm{H} & 1.58046 & -1.49963 & 2.84380 \\ \mathrm{H} & -1.23194 & 2.51828 & 1.30122 \\ \mathrm{H} & 0.53921 & 2.57055 & 1.27521 \\ \mathrm{H} & -0.38853 & 3.24669 & -0.07217 \\ \mathrm{O} & -1.68244 & -0.82870 & -0.62691 \\ \mathrm{O} & -2.52856 & 0.63311 & 0.86755 \\ \mathrm{C} & -2.90819 & -1.56859 & -0.50750 \\ \mathrm{H} & -2.79216 & -2.42709 & -1.17117 \\ \mathrm{H} & -3.06586 & -1.89620 & 0.52391 \\ \mathrm{H} & -3.75754 & -0.95430 & -0.81920\end{array}$

$\mathrm{E}(\mathrm{UB}+\mathrm{HF}-\mathrm{LYP})=-539.844595454$

$\mathrm{S} * * 2=0.3212$

1 imaginary frequency

$383 \mathrm{~K}, 1.00 \mathrm{~atm}$, freq's scaled by 1.0000

Zero-point correction $=0.216468$

Thermal correction to $\mathrm{U}=0.236871$

Thermal correction to $\mathrm{H}=0.238084$

Thermal correction to $\mathrm{G}=0.159961$ 


\section{TS-1d-5Ed-Cis}

UB3LYP / 6-31+G (d) :

$\begin{array}{lrrr}\text { C } & 4.68048 & -0.18714 & -0.40480 \\ \mathrm{C} & 3.83952 & -0.40508 & 0.64939 \\ \mathrm{C} & 2.49606 & 0.04508 & 0.77197 \\ \mathrm{C} & 1.75289 & 0.90721 & -0.21993 \\ \mathrm{C} & 0.47362 & 0.09407 & -0.33964 \\ \mathrm{C} & -0.80232 & 0.58628 & -0.13230 \\ \mathrm{C} & 0.88224 & -1.26834 & -0.43687 \\ \mathrm{C} & -1.10672 & 2.06386 & -0.02195 \\ \mathrm{C} & -1.95406 & -0.33590 & -0.04721 \\ \mathrm{H} & 1.62258 & 1.93504 & 0.13242 \\ \mathrm{H} & 2.27689 & 0.95885 & -1.18173 \\ \mathrm{H} & 4.21908 & -0.99252 & 1.48556 \\ \mathrm{H} & 4.38721 & 0.38753 & -1.27891 \\ \mathrm{H} & 2.01461 & -0.06667 & 1.73965 \\ \mathrm{H} & 0.20518 & -2.08891 & -0.23413 \\ \mathrm{H} & 1.80864 & -1.49919 & -0.95085 \\ \mathrm{H} & 5.69256 & -0.58010 & -0.40004 \\ \mathrm{O} & -3.12824 & 0.34658 & 0.09424 \\ \mathrm{O} & -1.93484 & -1.55941 & -0.09011 \\ \mathrm{C} & -4.31052 & -0.46167 & 0.18506 \\ \mathrm{H} & -5.13590 & 0.24443 & 0.28806 \\ \mathrm{H} & -4.43220 & -1.06801 & -0.71709 \\ \mathrm{H} & -4.25884 & -1.12345 & 1.05442 \\ \mathrm{H} & -1.75392 & 2.39471 & -0.84394 \\ \mathrm{H} & -1.64590 & 2.29591 & 0.90457 \\ \mathrm{H} & -0.20248 & 2.67474 & -0.05341\end{array}$

$\mathrm{E}(\mathrm{UB}+\mathrm{HF}-\mathrm{LYP})=-539.847107919$

$\mathrm{S} * * 2=0.4798$

1 imaginary frequency

$383 \mathrm{~K}, 1.00 \mathrm{~atm}$, freq's scaled by 1.0000

Zero-point correction $=0.216028$

Thermal correction to $U=0.236563$

Thermal correction to $\mathrm{H}=0.237776$

Thermal correction to $\mathrm{G}=0.159284$

\section{TS-1d-5Ed-trans}

UB3LYP/6-31+G (d) :

$\begin{array}{lrrr}\mathrm{C} & 4.51141 & -0.47577 & -0.45825 \\ \mathrm{C} & 3.67299 & -0.62466 & 0.60928 \\ \mathrm{C} & 2.38155 & -0.04730 & 0.76279 \\ \mathrm{C} & 1.70804 & 0.89813 & -0.20228 \\ \mathrm{C} & 0.35628 & 0.20756 & -0.31721 \\ \mathrm{C} & -0.83877 & 0.86833 & -0.10238 \\ \mathrm{C} & 0.63874 & -1.18509 & -0.41990 \\ \mathrm{C} & -0.87189 & 2.38015 & 0.00389 \\ \mathrm{C} & -2.16294 & 0.22018 & 0.01257 \\ \mathrm{H} & 1.66058 & 1.92522 & 0.17487 \\ \mathrm{H} & 2.22437 & 0.92936 & -1.16924 \\ \mathrm{H} & 4.00846 & -1.25720 & 1.43132 \\ \mathrm{H} & 4.26021 & 0.13740 & -1.31920 \\ \mathrm{H} & 1.91292 & -0.12126 & 1.74037 \\ \mathrm{H} & -0.10066 & -1.94870 & -0.21544 \\ \mathrm{H} & 1.52934 & -1.49621 & -0.95417 \\ \mathrm{H} & 5.48047 & -0.96498 & -0.47752 \\ \mathrm{O} & -2.14837 & -1.14049 & -0.05204 \\ \mathrm{O} & -3.21272 & 0.83337 & 0.15606 \\ \mathrm{C} & -3.43154 & -1.77930 & 0.05003 \\ \mathrm{H} & -3.22736 & -2.84845 & -0.02765 \\ \mathrm{H} & -3.90269 & -1.54577 & 1.00875 \\ \mathrm{H} & -4.08894 & -1.45310 & -0.76053 \\ \mathrm{H} & -1.90410 & 2.72777 & 0.06328 \\ \mathrm{H} & -0.34814 & 2.73767 & 0.90238 \\ \mathrm{H} & -0.39904 & 2.86185 & -0.86055\end{array}$

$\mathrm{E}(\mathrm{UB}+\mathrm{HF}-\mathrm{LYP})=-539.845110539$

$\mathrm{S} * * 2=0.4694$

1 imaginary frequency

$383 \mathrm{~K}, 1.00 \mathrm{~atm}$, freq's scaled by 1.0000

Zero-point correction $=0.215938$

Thermal correction to $\mathrm{U}=0.236539$

Thermal correction to $\mathrm{H}=0.237752$

Thermal correction to $G=0.158775$ 


\section{TS-1d-6Ed-Cis}

UB3LYP / 6-31+G (d) :

$\begin{array}{lrrr}\mathrm{C} & 2.02573 & -1.56319 & 1.41753 \\ \mathrm{C} & 2.74580 & -0.41012 & 1.29271 \\ \mathrm{C} & 2.85485 & 0.38271 & 0.11791 \\ \mathrm{C} & 2.12828 & 0.06187 & -1.17043 \\ \mathrm{C} & 0.76104 & 0.62060 & -0.77779 \\ \mathrm{C} & -0.39402 & -0.14293 & -0.70306 \\ \mathrm{C} & 0.99558 & 1.89507 & -0.20705 \\ \mathrm{H} & 1.82495 & 2.48833 & -0.57666 \\ \mathrm{H} & 0.25924 & 2.39578 & 0.40921 \\ \mathrm{H} & 2.56784 & 0.60113 & -2.01664 \\ \mathrm{H} & 2.13657 & -1.00292 & -1.41427 \\ \mathrm{H} & 3.27444 & -0.03895 & 2.17029 \\ \mathrm{H} & 1.49489 & -2.01302 & 0.58534 \\ \mathrm{H} & 3.61947 & 1.15374 & 0.10240 \\ \mathrm{C} & -0.54815 & -1.45882 & -1.43456 \\ \mathrm{C} & -1.56818 & 0.34243 & 0.04877 \\ \mathrm{H} & 1.97158 & -2.08519 & 2.36822 \\ \mathrm{H} & -0.80800 & -2.28104 & -0.75644 \\ \mathrm{H} & -1.36219 & -1.40057 & -2.16785 \\ \mathrm{H} & 0.35673 & -1.73386 & -1.98087 \\ \mathrm{O} & -2.61081 & -0.53691 & -0.03673 \\ \mathrm{O} & -1.66961 & 1.38192 & 0.68823 \\ \mathrm{C} & -3.80007 & -0.16125 & 0.67222 \\ \mathrm{H} & -4.50996 & -0.97081 & 0.49522 \\ \mathrm{H} & -3.59386 & -0.05660 & 1.74133 \\ \mathrm{H} & -4.19342 & 0.78572 & 0.29139\end{array}$

$\mathrm{E}(\mathrm{UB}+\mathrm{HF}-\mathrm{LYP})=-539.844836933$

$\mathrm{S} * * 2=0.3710$

1 imaginary frequency

$383 \mathrm{~K}, 1.00 \mathrm{~atm}$, freq's scaled by 1.0000

Zero-point correction $=0.216546$

Thermal correction to $U=0.236816$

Thermal correction to $\mathrm{H}=0.238029$

Thermal correction to $\mathrm{G}=0.160667$

\section{TS-1d-6Ed-trans}

UB3LYP / 6-31+G (d) :

$\begin{array}{lrrr}\mathrm{C} & -2.13346 & 0.26230 & 1.97546 \\ \mathrm{C} & -2.63505 & -0.72874 & 1.18140 \\ \mathrm{C} & -2.65884 & -0.72674 & -0.24000 \\ \mathrm{C} & -2.08085 & 0.39425 & -1.07568 \\ \mathrm{C} & -0.60680 & -0.00956 & -0.98181 \\ \mathrm{C} & 0.37307 & 0.80598 & -0.43836 \\ \mathrm{C} & -0.57088 & -1.40105 & -1.24364 \\ \mathrm{H} & -1.29329 & -1.81756 & -1.93691 \\ \mathrm{H} & 0.27432 & -2.02577 & -0.98666 \\ \mathrm{H} & -2.45550 & 0.35331 & -2.10440 \\ \mathrm{H} & -2.30920 & 1.38549 & -0.67850 \\ \mathrm{H} & -3.03868 & -1.61794 & 1.66511 \\ \mathrm{H} & -1.73855 & 1.18925 & 1.57352 \\ \mathrm{H} & -3.26699 & -1.47997 & -0.73236 \\ \mathrm{C} & 0.20982 & 2.30669 & -0.32930 \\ \mathrm{C} & 1.70273 & 0.35865 & 0.02600 \\ \mathrm{H} & -2.12455 & 0.15817 & 3.05637 \\ \mathrm{H} & 0.98683 & 2.81429 & -0.91370 \\ \mathrm{H} & -0.75730 & 2.65069 & -0.70059 \\ \mathrm{H} & 0.33785 & 2.65546 & 0.70311 \\ \mathrm{O} & 1.92542 & -0.98487 & -0.00759 \\ \mathrm{O} & 2.56102 & 1.13394 & 0.42739 \\ \mathrm{C} & 3.21729 & -1.40835 & 0.45745 \\ \mathrm{H} & 3.21856 & -2.49469 & 0.35397 \\ \mathrm{H} & 4.01035 & -0.96239 & -0.14906 \\ \mathrm{H} & 3.36246 & -1.12149 & 1.50261\end{array}$

$\mathrm{E}(\mathrm{UB}+\mathrm{HF}-\mathrm{LYP})=-539.842876507$

$\mathrm{S} * * 2=0.3719$

1 imaginary frequency

$383 \mathrm{~K}, 1.00 \mathrm{~atm}$, freq's scaled by 1.0000

Zero-point correction $=0.216405$

Thermal correction to $\mathrm{U}=0.236824$

Thermal correction to $\mathrm{H}=0.238037$

Thermal correction to $\mathrm{G}=0.159730$ 
TS-Zd-2d-x-cis

UB3LYP / 6-31+G (d) :

$\begin{array}{lrrr}\text { C } & -0.17405 & 0.78131 & 0.23708 \\ \mathrm{C} & 1.06799 & 0.93889 & -0.47434 \\ \mathrm{C} & 1.81006 & 2.07479 & -0.37784 \\ \mathrm{C} & 1.72070 & -0.24702 & -1.22967 \\ \mathrm{C} & 2.62696 & -0.89982 & -0.23294 \\ \mathrm{C} & 2.13240 & -1.52346 & 0.90373 \\ \mathrm{C} & 0.78096 & -1.80083 & 1.09732 \\ \mathrm{H} & 2.77330 & 2.15828 & -0.87441 \\ \mathrm{H} & 1.45485 & 2.95827 & 0.14448 \\ \mathrm{H} & 2.28838 & 0.12765 & -2.08582 \\ \mathrm{H} & 0.94248 & -0.91373 & -1.60910 \\ \mathrm{H} & 2.82844 & -1.74613 & 1.71258 \\ \mathrm{H} & 0.09369 & -1.87107 & 0.26552 \\ \mathrm{H} & 3.69895 & -0.74065 & -0.32622 \\ \mathrm{C} & -1.28150 & 0.04650 & -0.37865 \\ \mathrm{C} & -0.44638 & 1.61311 & 1.46773 \\ \mathrm{H} & 0.42106 & -2.15796 & 2.05832 \\ \mathrm{O} & -2.42692 & 0.11161 & 0.36341 \\ \mathrm{O} & -1.24858 & -0.56968 & -1.44201 \\ \mathrm{C} & -3.56541 & -0.56365 & -0.19052 \\ \mathrm{H} & -4.37833 & -0.38400 & 0.51477 \\ \mathrm{H} & -3.81290 & -0.15787 & -1.17556 \\ \mathrm{H} & -3.37046 & -1.63581 & -0.28632 \\ \mathrm{H} & -1.18399 & 1.13372 & 2.11420 \\ \mathrm{H} & 0.47210 & 1.77207 & 2.04013 \\ \mathrm{H} & -0.85393 & 2.60281 & 1.20477\end{array}$

$\mathrm{E}(\mathrm{UB}+\mathrm{HF}-\mathrm{LYP})=-539.853081368$

$\mathrm{S} * * 2=0.5217$

1 imaginary frequency

$383 \mathrm{~K}, 1.00 \mathrm{~atm}$, freq's scaled by 1.0000

Zero-point correction $=0.216623$

Thermal correction to $U=0.236685$

Thermal correction to $\mathrm{H}=0.237898$

Thermal correction to $\mathrm{G}=0.161919$

\section{TS-Zd-2d-x-trans}

UB3LYP/ $6-31+G(d)$ :

$\begin{array}{lrrr}\mathrm{C} & -0.13325 & 0.96315 & 0.12772 \\ \mathrm{C} & 0.88313 & 0.65207 & -0.84752 \\ \mathrm{C} & 1.66230 & 1.62521 & -1.39005 \\ \mathrm{C} & 1.28895 & -0.80942 & -1.17052 \\ \mathrm{C} & 2.38341 & -1.15131 & -0.20598 \\ \mathrm{C} & 2.15111 & -1.23789 & 1.15987 \\ \mathrm{C} & 0.87979 & -1.24294 & 1.72790 \\ \mathrm{H} & 2.46678 & 1.38167 & -2.07954 \\ \mathrm{H} & 1.48675 & 2.68034 & -1.20255 \\ \mathrm{H} & 1.64673 & -0.87145 & -2.20208 \\ \mathrm{H} & 0.42475 & -1.46999 & -1.07902 \\ \mathrm{H} & 3.01276 & -1.23338 & 1.82761 \\ \mathrm{H} & 0.00154 & -1.50634 & 1.15453 \\ \mathrm{H} & 3.40719 & -1.16881 & -0.57239 \\ \mathrm{C} & -1.45322 & 0.32251 & 0.17131 \\ \mathrm{C} & -0.00710 & 2.20334 & 0.97628 \\ \mathrm{H} & 0.74757 & -1.15880 & 2.80260 \\ \mathrm{O} & -1.63136 & -0.66371 & -0.76025 \\ \mathrm{O} & -2.35204 & 0.64012 & 0.94127 \\ \mathrm{C} & -2.92901 & -1.28091 & -0.76928 \\ \mathrm{H} & -2.88630 & -2.03021 & -1.56130 \\ \mathrm{H} & -3.14298 & -1.75049 & 0.19508 \\ \mathrm{H} & -3.70324 & -0.53902 & -0.98358 \\ \mathrm{H} & -0.65112 & 2.13053 & 1.85533 \\ \mathrm{H} & 1.02798 & 2.35946 & 1.29395 \\ \mathrm{H} & -0.32800 & 3.10227 & 0.42477\end{array}$

$\mathrm{E}(\mathrm{UB}+\mathrm{HF}-\mathrm{LYP})=-539.851183018$

$\mathrm{S} * * 2=0.5886$

1 imaginary frequency

$383 \mathrm{~K}, 1.00 \mathrm{~atm}$, freq's scaled by 1.0000

Zero-point correction $=0.216289$

Thermal correction to $\mathrm{U}=0.236476$

Thermal correction to $\mathrm{H}=0.237689$

Thermal correction to $\mathrm{G}=0.161318$ 


\section{TS-Zd-2d-n-cis}

UB3LYP / 6-31+G (d) :

$\begin{array}{lrrr}\text { C } & -0.91246 & -0.91059 & 1.94602 \\ \mathrm{C} & -0.86145 & -1.95214 & 1.01931 \\ \mathrm{C} & -1.28191 & -1.87653 & -0.29693 \\ \mathrm{C} & -1.82984 & -0.65991 & -0.98347 \\ \mathrm{C} & -1.38200 & 0.72989 & -0.49421 \\ \mathrm{C} & -0.06054 & 0.97085 & 0.04270 \\ \mathrm{C} & -2.30088 & 1.73268 & -0.56069 \\ \mathrm{C} & 1.04540 & 0.13689 & -0.40385 \\ \mathrm{C} & 0.22254 & 2.20689 & 0.85747 \\ \mathrm{H} & -1.60231 & -0.75324 & -2.05211 \\ \mathrm{H} & -2.92954 & -0.67344 & -0.90527 \\ \mathrm{H} & -0.35782 & -2.87007 & 1.32243 \\ \mathrm{H} & -1.56693 & -0.05786 & 1.83229 \\ \mathrm{H} & -1.17037 & -2.76060 & -0.91874 \\ \mathrm{H} & -3.30625 & 1.54732 & -0.93008 \\ \mathrm{H} & -2.06555 & 2.75790 & -0.29395 \\ \mathrm{H} & -0.41824 & -1.01466 & 2.90873 \\ \mathrm{O} & 2.23918 & 0.44953 & 0.18356 \\ \mathrm{O} & 0.95525 & -0.76696 & -1.23483 \\ \mathrm{C} & 3.36354 & -0.32895 & -0.24600 \\ \mathrm{H} & 4.22135 & 0.08226 & 0.28873 \\ \mathrm{H} & 3.22377 & -1.38372 & 0.00916 \\ \mathrm{H} & 3.50569 & -0.24037 & -1.32719 \\ \mathrm{H} & 1.12926 & 2.08443 & 1.45226 \\ \mathrm{H} & 0.37704 & 3.08640 & 0.21200 \\ \mathrm{H} & -0.60996 & 2.44059 & 1.52978\end{array}$

$\mathrm{E}(\mathrm{UB}+\mathrm{HF}-\mathrm{LYP})=-539.851583199$

$\mathrm{S} * * 2=0.6081$

1 imaginary frequency

$383 \mathrm{~K}, 1.00 \mathrm{~atm}$, freq's scaled by 1.0000

Zero-point correction $=0.216204$

Thermal correction to $\mathrm{U}=0.236293$

Thermal correction to $\mathrm{H}=0.237506$

Thermal correction to $\mathrm{G}=0.161893$

\section{TS-Zd-2d-n-trans}

UB3LYP/ $6-31+G(d):$

$\begin{array}{lrrr}\mathrm{C} & -0.97863 & -0.80388 & 2.00727 \\ \mathrm{C} & -0.31212 & -1.85603 & 1.36475 \\ \mathrm{C} & -0.36521 & -2.14110 & 0.01606 \\ \mathrm{C} & -1.05864 & -1.32790 & -1.04013 \\ \mathrm{C} & -1.30531 & 0.16986 & -0.78057 \\ \mathrm{C} & -0.40777 & 0.99985 & -0.00421 \\ \mathrm{C} & -2.45762 & 0.69371 & -1.28415 \\ \mathrm{C} & 1.04873 & 0.80727 & 0.04464 \\ \mathrm{C} & -0.88653 & 2.29982 & 0.58734 \\ \mathrm{H} & -0.49307 & -1.44268 & -1.97348 \\ \mathrm{H} & -2.04808 & -1.77083 & -1.23878 \\ \mathrm{H} & 0.37235 & -2.45279 & 1.96867 \\ \mathrm{H} & -1.85961 & -0.33344 & 1.59366 \\ \mathrm{H} & 0.19856 & -2.99819 & -0.34580 \\ \mathrm{H} & -3.16054 & 0.07404 & -1.83527 \\ \mathrm{H} & -2.70697 & 1.74622 & -1.19873 \\ \mathrm{H} & -0.73646 & -0.55608 & 3.03730 \\ \mathrm{O} & 1.49585 & -0.16268 & -0.80190 \\ \mathrm{O} & 1.81883 & 1.46874 & 0.73024 \\ \mathrm{C} & 2.91404 & -0.38958 & -0.78732 \\ \mathrm{H} & 3.08680 & -1.19268 & -1.50546 \\ \mathrm{H} & 3.44865 & 0.51620 & -1.08664 \\ \mathrm{H} & 3.24596 & -0.68584 & 0.21174 \\ \mathrm{H} & -0.19860 & 2.63094 & 1.36812 \\ \mathrm{H} & -0.91879 & 3.09785 & -0.17171 \\ \mathrm{H} & -1.89319 & 2.20666 & 1.00757\end{array}$

$\mathrm{E}(\mathrm{UB}+\mathrm{HF}-\mathrm{LYP})=-539.848197366$

$\mathrm{S} * * 2=0.7078$

1 imaginary frequency

$383 \mathrm{~K}, 1.00 \mathrm{~atm}$, freq's scaled by 1.0000

Zero-point correction $=0.216036$

Thermal correction to $\mathrm{U}=0.236146$

Thermal correction to $\mathrm{H}=0.237359$

Thermal correction to $\mathrm{G}=0.161718$ 
TS ' $-Z d-2 d-x-c i s$

UB3LYP / 6-31+G (d) :

$\begin{array}{rrrr}\mathrm{C} & -1.86797 & 1.62639 & -0.47094 \\ \mathrm{C} & -0.84383 & 0.68705 & -0.64399 \\ \mathrm{C} & 0.45984 & 0.89313 & -0.18129 \\ \mathrm{C} & -1.30078 & -0.68597 & -1.17182 \\ \mathrm{C} & -1.52285 & -1.55546 & 0.03743 \\ \mathrm{C} & -2.44210 & -1.21604 & 1.00572 \\ \mathrm{C} & -3.39217 & -0.19090 & 0.85242 \\ \mathrm{H} & -0.56248 & -1.13350 & -1.83418 \\ \mathrm{H} & -2.23066 & -0.53599 & -1.73765 \\ \mathrm{H} & -2.36993 & -1.70513 & 1.97802 \\ \mathrm{H} & -3.81446 & 0.04232 & -0.11499 \\ \mathrm{H} & -0.82689 & -2.37087 & 0.20992 \\ \mathrm{H} & -3.93817 & 0.18877 & 1.71202 \\ \mathrm{H} & -1.74575 & 2.52391 & 0.12367 \\ \mathrm{H} & -2.75752 & 1.58358 & -1.08761 \\ \mathrm{C} & 1.49287 & -0.17086 & -0.23052 \\ \mathrm{O} & 1.44769 & -1.23914 & -0.82600 \\ \mathrm{O} & 2.59771 & 0.18671 & 0.48456 \\ \mathrm{C} & 0.90612 & 2.22699 & 0.37508 \\ \mathrm{H} & 0.24169 & 3.03653 & 0.06919 \\ \mathrm{H} & 0.93879 & 2.20687 & 1.47376 \\ \mathrm{H} & 1.91750 & 2.47616 & 0.04117 \\ \mathrm{C} & 3.67082 & -0.76694 & 0.49273 \\ \mathrm{H} & 4.45221 & -0.31590 & 1.10599 \\ \mathrm{H} & 3.33968 & -1.71484 & 0.92655 \\ \mathrm{H} & 4.03302 & -0.94687 & -0.52336 \\ & & & \end{array}$

$\mathrm{E}(\mathrm{UB}+\mathrm{HF}-\mathrm{LYP})=-539.848347352$

$\mathrm{S} * * 2=0.5456$

1 imaginary frequency

$383 \mathrm{~K}, 1.00 \mathrm{~atm}$, freq's scaled by 1.0000

Zero-point correction $=0.216507$

Thermal correction to $\mathrm{U}=0.236582$

Thermal correction to $\mathrm{H}=0.237795$

Thermal correction to $\mathrm{G}=0.160541$

\section{TS ' $-z d-2 d-x-t r a n s$}

UB3LYP / 6-31+G (d) :

$\begin{array}{rrrr}\mathrm{C} & -1.92670 & 1.33479 & -0.84941 \\ \mathrm{C} & -0.76074 & 0.56980 & -0.73728 \\ \mathrm{C} & 0.38881 & 1.08792 & -0.13015 \\ \mathrm{C} & -0.92370 & -0.92486 & -1.06148 \\ \mathrm{C} & -1.15767 & -1.61301 & 0.25933 \\ \mathrm{C} & -2.22672 & -1.27475 & 1.05961 \\ \mathrm{C} & -3.29489 & -0.46688 & 0.62995 \\ \mathrm{H} & -0.05384 & -1.33645 & -1.56837 \\ \mathrm{H} & -1.78648 & -1.03538 & -1.73260 \\ \mathrm{H} & -2.20049 & -1.57359 & 2.10816 \\ \mathrm{H} & -3.62881 & -0.47975 & -0.39820 \\ \mathrm{H} & -0.38161 & -2.27039 & 0.64184 \\ \mathrm{H} & -3.99393 & -0.04922 & 1.34961 \\ \mathrm{H} & -2.01016 & 2.32668 & -0.42084 \\ \mathrm{H} & -2.70813 & 1.04862 & -1.54332 \\ \mathrm{C} & 1.63019 & 0.33888 & 0.18896 \\ \mathrm{O} & 2.46000 & 0.73882 & 0.99389 \\ \mathrm{O} & 1.82308 & -0.81561 & -0.50399 \\ \mathrm{C} & 0.43551 & 2.53783 & 0.30166 \\ \mathrm{H} & 0.16519 & 3.20635 & -0.52364 \\ \mathrm{H} & -0.26995 & 2.73028 & 1.12293 \\ \mathrm{H} & 1.43148 & 2.80253 & 0.65787 \\ \mathrm{C} & 3.03423 & -1.52688 & -0.19726 \\ \mathrm{H} & 3.01766 & -2.41089 & -0.83655 \\ \mathrm{H} & 3.90873 & -0.90875 & -0.41771 \\ \mathrm{H} & 3.05580 & -1.81275 & 0.85815\end{array}$

$\mathrm{E}(\mathrm{UB}+\mathrm{HF}-\mathrm{LYP})=-539.846394504$

$\mathrm{S} * * 2=0.5266$

1 imaginary frequency

$383 \mathrm{~K}, 1.00 \mathrm{~atm}$, freq's scaled by 1.0000

Zero-point correction $=0.216600$

Thermal correction to $\mathrm{U}=0.236599$

Thermal correction to $\mathrm{H}=0.237812$

Thermal correction to $\mathrm{G}=0.161147$ 
TS ' - Zd-2d-n-cis

UB3LYP / 6-31+G (d) :

$\begin{array}{rrrr}\mathrm{C} & 3.07556 & 0.29573 & 1.35131 \\ \mathrm{C} & 3.51713 & -0.62301 & 0.37545 \\ \mathrm{C} & 2.66818 & -1.25751 & -0.49944 \\ \mathrm{C} & 1.17983 & -1.04625 & -0.50387 \\ \mathrm{C} & 0.70172 & 0.41905 & -0.44264 \\ \mathrm{C} & 1.65772 & 1.44504 & -0.54076 \\ \mathrm{C} & -0.64969 & 0.73986 & -0.24173 \\ \mathrm{H} & 0.73421 & -1.53659 & -1.37461 \\ \mathrm{H} & 0.73503 & -1.56889 & 0.35366 \\ \mathrm{H} & 4.59158 & -0.74919 & 0.24102 \\ \mathrm{H} & 2.07789 & 0.25206 & 1.76932 \\ \mathrm{H} & 3.08343 & -1.94193 & -1.23557 \\ \mathrm{H} & 3.78986 & 0.92000 & 1.88189 \\ \mathrm{H} & 1.38005 & 2.48206 & -0.39230 \\ \mathrm{H} & 2.63670 & 1.26895 & -0.95793 \\ \mathrm{C} & -1.67692 & -0.30945 & -0.06816 \\ \mathrm{O} & -1.53216 & -1.51521 & -0.22765 \\ \mathrm{O} & -2.88639 & 0.20531 & 0.30150 \\ \mathrm{C} & -1.10331 & 2.18668 & -0.22199 \\ \mathrm{H} & -0.70515 & 2.72158 & 0.65234 \\ \mathrm{H} & -0.75871 & 2.72325 & -1.11378 \\ \mathrm{H} & -2.18898 & 2.26166 & -0.18145 \\ \mathrm{C} & -3.93854 & -0.75326 & 0.48570 \\ \mathrm{H} & -4.13152 & -1.29639 & -0.44379 \\ \mathrm{H} & -3.67387 & -1.46968 & 1.26863 \\ \mathrm{H} & -4.81208 & -0.16895 & 0.77858 \\ & & & \end{array}$

$\mathrm{E}(\mathrm{UB}+\mathrm{HF}-\mathrm{LYP})=-539.848031767$

$\mathrm{S} * * 2=0.6188$

1 imaginary frequency

$383 \mathrm{~K}, 1.00 \mathrm{~atm}$, freq's scaled by 1.0000

Zero-point correction $=0.216333$

Thermal correction to $U=0.236433$

Thermal correction to $\mathrm{H}=0.237646$

Thermal correction to $\mathrm{G}=0.160436$

\section{TS ' -zd-2d-n-trans}

UB3LYP / 6-31+G (d) :

$\begin{array}{lrrr}\mathrm{C} & 3.05244 & 0.23395 & 1.21037 \\ \mathrm{C} & 3.30494 & -0.85693 & 0.35098 \\ \mathrm{C} & 2.33721 & -1.47555 & -0.40321 \\ \mathrm{C} & 0.88968 & -1.06367 & -0.41303 \\ \mathrm{C} & 0.60990 & 0.45248 & -0.46869 \\ \mathrm{C} & 1.68077 & 1.31643 & -0.75372 \\ \mathrm{C} & -0.65485 & 0.98785 & -0.18247 \\ \mathrm{H} & 0.37600 & -1.55799 & -1.24334 \\ \mathrm{H} & 0.40019 & -1.45380 & 0.49045 \\ \mathrm{H} & 4.34257 & -1.15698 & 0.20253 \\ \mathrm{H} & 2.08425 & 0.39411 & 1.66690 \\ \mathrm{H} & 2.62197 & -2.30913 & -1.04117 \\ \mathrm{H} & 3.87797 & 0.80547 & 1.62673 \\ \mathrm{H} & 1.55465 & 2.39208 & -0.72149 \\ \mathrm{H} & 2.59107 & 0.95547 & -1.20710 \\ \mathrm{C} & -1.87230 & 0.21063 & 0.15441 \\ \mathrm{O} & -2.85806 & 0.69962 & 0.69055 \\ \mathrm{O} & -1.84806 & -1.09838 & -0.21255 \\ \mathrm{C} & -0.88423 & 2.48570 & -0.18308 \\ \mathrm{H} & -0.23634 & 2.99704 & 0.54146 \\ \mathrm{H} & -0.67854 & 2.92238 & -1.16871 \\ \mathrm{H} & -1.91768 & 2.70811 & 0.08384 \\ \mathrm{C} & -3.03288 & -1.85168 & 0.09649 \\ \mathrm{H} & -3.21909 & -1.85133 & 1.17401 \\ \mathrm{H} & -3.90074 & -1.42780 & -0.41617 \\ \mathrm{H} & -2.83132 & -2.86282 & -0.26015 \\ & & & \end{array}$

$\mathrm{E}(\mathrm{UB}+\mathrm{HF}-\mathrm{LYP})=-539.846338967$

$\mathrm{S} * * 2=0.6402$

1 imaginary frequency

$383 \mathrm{~K}, 1.00 \mathrm{~atm}$, freq's scaled by 1.0000

Zero-point correction $=0.216182$

Thermal correction to $\mathrm{U}=0.236333$

Thermal correction to $\mathrm{H}=0.237546$

Thermal correction to $\mathrm{G}=0.160210$ 


\section{TS-Ed-2d-x-cis}

UB3LYP / 6-31+G (d) :

$\begin{array}{lrrr}\text { C } & 0.19972 & 0.27454 & 0.68650 \\ \mathrm{C} & -0.97393 & 1.03228 & 0.34477 \\ \mathrm{C} & -0.96433 & 2.16966 & -0.39866 \\ \mathrm{C} & -2.37078 & 0.44948 & 0.69094 \\ \mathrm{C} & -2.81358 & -0.26129 & -0.54972 \\ \mathrm{C} & -2.14255 & -1.37418 & -1.04208 \\ \mathrm{C} & -1.15061 & -2.05557 & -0.34624 \\ \mathrm{H} & -1.89566 & 2.66666 & -0.66109 \\ \mathrm{H} & -0.04298 & 2.62893 & -0.73546 \\ \mathrm{H} & -3.06440 & 1.25408 & 0.94993 \\ \mathrm{H} & -2.31456 & -0.22131 & 1.55471 \\ \mathrm{H} & -2.35497 & -1.68238 & -2.06546 \\ \mathrm{H} & -1.03747 & -1.97184 & 0.72577 \\ \mathrm{H} & -3.57380 & 0.21218 & -1.16617 \\ \mathrm{C} & 0.36854 & -0.32307 & 2.06221 \\ \mathrm{C} & 1.36797 & 0.30084 & -0.20557 \\ \mathrm{H} & -0.55867 & -2.82481 & -0.83395 \\ \mathrm{H} & 0.87203 & -1.29417 & 2.04055 \\ \mathrm{H} & 0.99145 & 0.33673 & 2.68483 \\ \mathrm{H} & -0.58803 & -0.43202 & 2.58201 \\ \mathrm{O} & 2.43448 & -0.35940 & 0.34187 \\ \mathrm{O} & 1.44172 & 0.83050 & -1.30733 \\ \mathrm{C} & 3.61566 & -0.40665 & -0.47037 \\ \mathrm{H} & 4.34556 & -0.97065 & 0.11266 \\ \mathrm{H} & 3.41054 & -0.90904 & -1.42037 \\ \mathrm{H} & 3.98466 & 0.60263 & -0.67495\end{array}$

$\mathrm{E}(\mathrm{UB}+\mathrm{HF}-\mathrm{LYP})=-539.850101737$

$\mathrm{S} * * 2=0.5590$

1 imaginary frequency

$383 \mathrm{~K}, 1.00 \mathrm{~atm}$, freq's scaled by 1.0000

Zero-point correction $=0.216230$

Thermal correction to $U=0.236391$

Thermal correction to $\mathrm{H}=0.237604$

Thermal correction to $\mathrm{G}=0.161452$
TS-Ed-2d-x-trans

UB3LYP / 6-31+G (d) :

$\begin{array}{lrrr}\mathrm{C} & 0.16828 & 0.82997 & -0.44281 \\ \mathrm{C} & -0.77909 & -0.01423 & -1.12840 \\ \mathrm{C} & -0.44683 & -1.01044 & -1.98694 \\ \mathrm{C} & -2.28898 & 0.13232 & -0.79094 \\ \mathrm{C} & -2.57532 & -0.88807 & 0.26575 \\ \mathrm{C} & -2.00971 & -0.82051 & 1.53391 \\ \mathrm{C} & -1.30311 & 0.27364 & 2.01864 \\ \mathrm{H} & -1.21589 & -1.62289 & -2.45241 \\ \mathrm{H} & 0.58091 & -1.21817 & -2.26120 \\ \mathrm{H} & -2.89298 & -0.05187 & -1.68360 \\ \mathrm{H} & -2.51380 & 1.14900 & -0.45182 \\ \mathrm{H} & -2.06920 & -1.70931 & 2.16190 \\ \mathrm{H} & -1.37254 & 1.25339 & 1.56606 \\ \mathrm{H} & -3.10927 & -1.78905 & -0.02644 \\ \mathrm{C} & -0.04276 & 2.31963 & -0.35415 \\ \mathrm{C} & 1.49722 & 0.38287 & -0.00291 \\ \mathrm{H} & -0.76677 & 0.21636 & 2.96139 \\ \mathrm{H} & 0.37576 & 2.73593 & 0.56673 \\ \mathrm{H} & 0.47908 & 2.81906 & -1.18480 \\ \mathrm{H} & -1.09806 & 2.59801 & -0.43251 \\ \mathrm{O} & 1.70212 & -0.96110 & -0.07411 \\ \mathrm{O} & 2.36189 & 1.14715 & 0.41390 \\ \mathrm{C} & 2.98203 & -1.41388 & 0.38974 \\ \mathrm{H} & 2.97228 & -2.49630 & 0.25198 \\ \mathrm{H} & 3.78918 & -0.95768 & -0.19076 \\ \mathrm{H} & 3.11877 & -1.16275 & 1.44586\end{array}$

$\mathrm{E}(\mathrm{UB}+\mathrm{HF}-\mathrm{LYP})=-539.849246692$

$\mathrm{S} * * 2=0.6107$

1 imaginary frequency

$383 \mathrm{~K}, 1.00 \mathrm{~atm}$, freq's scaled by 1.0000

Zero-point correction $=0.216030$

Thermal correction to $\mathrm{U}=0.236326$

Thermal correction to $\mathrm{H}=0.237539$

Thermal correction to $\mathrm{G}=0.160808$ 


\section{TS-Ed-2d-n-cis}

UB3LYP / 6-31+G (d) :

$\begin{array}{lrrr}\mathrm{C} & 1.01935 & -1.20196 & -1.54952 \\ \mathrm{C} & 2.15293 & -1.52136 & -0.78800 \\ \mathrm{C} & 2.81235 & -0.68341 & 0.08994 \\ \mathrm{C} & 2.40245 & 0.71432 & 0.46759 \\ \mathrm{C} & 0.93791 & 1.11908 & 0.23378 \\ \mathrm{C} & -0.10356 & 0.15959 & 0.56220 \\ \mathrm{C} & 0.69285 & 2.36175 & -0.25324 \\ \mathrm{C} & 0.11129 & -0.76137 & 1.72585 \\ \mathrm{C} & -1.42760 & 0.25278 & -0.07047 \\ \mathrm{H} & 2.67015 & 0.86691 & 1.52476 \\ \mathrm{H} & 3.02262 & 1.43390 & -0.08909 \\ \mathrm{H} & 2.49940 & -2.55498 & -0.82649 \\ \mathrm{H} & 0.74460 & -0.19109 & -1.81333 \\ \mathrm{H} & 3.68524 & -1.07503 & 0.60918 \\ \mathrm{H} & 1.51575 & 3.03625 & -0.47951 \\ \mathrm{H} & -0.31155 & 2.72630 & -0.42721 \\ \mathrm{H} & 0.48916 & -1.99390 & -2.07319 \\ \mathrm{O} & -2.34605 & -0.56721 & 0.52175 \\ \mathrm{O} & -1.72772 & 0.95950 & -1.02622 \\ \mathrm{C} & -3.65364 & -0.55867 & -0.06801 \\ \mathrm{H} & -4.24106 & -1.26365 & 0.52230 \\ \mathrm{H} & -4.09197 & 0.44229 & -0.01993 \\ \mathrm{H} & -3.60722 & -0.87670 & -1.11376 \\ \mathrm{H} & -0.68252 & -1.50258 & 1.81629 \\ \mathrm{H} & 1.07593 & -1.28186 & 1.63895 \\ \mathrm{H} & 0.15562 & -0.18859 & 2.66614 \\ & & & \end{array}$

$\mathrm{E}(\mathrm{UB}+\mathrm{HF}-\mathrm{LYP})=-539.849192567$

$\mathrm{S} * * 2=0.6449$

1 imaginary frequency

$383 \mathrm{~K}, 1.00 \mathrm{~atm}$, freq's scaled by 1.0000

Zero-point correction $=0.216116$

Thermal correction to $U=0.236146$

Thermal correction to $\mathrm{H}=0.237359$

Thermal correction to $\mathrm{G}=0.161643$

\section{TS-Ed-2d-n-trans}

UB3LYP / 6-31+G (d) :

$\begin{array}{lrrr}\mathrm{C} & 0.92609 & -0.99123 & -1.68237 \\ \mathrm{C} & 2.21494 & -1.12391 & -1.14943 \\ \mathrm{C} & 2.84140 & -0.23111 & -0.30157 \\ \mathrm{C} & 2.23577 & 1.01609 & 0.28048 \\ \mathrm{C} & 0.70039 & 1.10574 & 0.36644 \\ \mathrm{C} & -0.03679 & -0.08707 & 0.75279 \\ \mathrm{C} & 0.12972 & 2.31146 & 0.12321 \\ \mathrm{C} & 0.58015 & -1.01738 & 1.75179 \\ \mathrm{C} & -1.44850 & -0.34386 & 0.42590 \\ \mathrm{H} & 2.67100 & 1.16545 & 1.28047 \\ \mathrm{H} & 2.56740 & 1.88654 & -0.30713 \\ \mathrm{H} & 2.74235 & -2.05690 & -1.35171 \\ \mathrm{H} & 0.42253 & -0.04061 & -1.78160 \\ \mathrm{H} & 3.85179 & -0.46447 & 0.02883 \\ \mathrm{H} & 0.74257 & 3.16879 & -0.14631 \\ \mathrm{H} & -0.93786 & 2.47478 & 0.20201 \\ \mathrm{H} & 0.46978 & -1.83325 & -2.19694 \\ \mathrm{O} & -1.96302 & 0.46523 & -0.54331 \\ \mathrm{O} & -2.12280 & -1.22307 & 0.95014 \\ \mathrm{C} & -3.32764 & 0.20382 & -0.90533 \\ \mathrm{H} & -3.56756 & 0.93965 & -1.67450 \\ \mathrm{H} & -3.43342 & -0.81149 & -1.29860 \\ \mathrm{H} & -3.98648 & 0.32033 & -0.04019 \\ \mathrm{H} & -0.05320 & -1.88949 & 1.92033 \\ \mathrm{H} & 1.57678 & -1.34700 & 1.42358 \\ \mathrm{H} & 0.72773 & -0.50486 & 2.71562\end{array}$

$\mathrm{E}(\mathrm{UB}+\mathrm{HF}-\mathrm{LYP})=-539.847985420$

$\mathrm{S} * * 2=0.6514$

1 imaginary frequency

$383 \mathrm{~K}, 1.00 \mathrm{~atm}$, freq's scaled by 1.0000

Zero-point correction $=0.215858$

Thermal correction to $\mathrm{U}=0.235998$

Thermal correction to $\mathrm{H}=0.237211$

Thermal correction to $\mathrm{G}=0.161193$ 
TS ' -Ed-2d-x-Cis

UB3LYP / 6-31+G (d) :

$\begin{array}{rrrr}\text { C } & 0.92658 & -1.50766 & -0.79284 \\ \mathrm{C} & 0.69852 & -0.12638 & -0.73897 \\ \mathrm{C} & -0.48407 & 0.48490 & -0.31378 \\ \mathrm{C} & 1.93361 & 0.75407 & -1.02575 \\ \mathrm{C} & 2.59140 & 1.08380 & 0.28849 \\ \mathrm{C} & 3.02847 & 0.09247 & 1.13815 \\ \mathrm{C} & 3.14160 & -1.26177 & 0.77386 \\ \mathrm{H} & 1.67117 & 1.66410 & -1.57131 \\ \mathrm{H} & 2.60579 & 0.18155 & -1.67982 \\ \mathrm{H} & 3.22619 & 0.35876 & 2.17747 \\ \mathrm{H} & 3.39110 & -1.55154 & -0.23720 \\ \mathrm{H} & 2.57807 & 2.11538 & 0.63184 \\ \mathrm{H} & 3.27323 & -2.02778 & 1.53310 \\ \mathrm{H} & 0.21817 & -2.21661 & -0.38871 \\ \mathrm{H} & 1.71149 & -1.89693 & -1.43121 \\ \mathrm{C} & -1.71252 & -0.31770 & -0.08638 \\ \mathrm{O} & -1.85609 & -1.52290 & -0.23238 \\ \mathrm{O} & -2.74473 & 0.46956 & 0.33433 \\ \mathrm{C} & -0.62967 & 1.97542 & -0.10684 \\ \mathrm{H} & -0.94411 & 2.19743 & 0.92011 \\ \mathrm{H} & 0.29882 & 2.51638 & -0.29007 \\ \mathrm{H} & -1.40777 & 2.39230 & -0.75865 \\ \mathrm{C} & -3.98335 & -0.21149 & 0.58530 \\ \mathrm{H} & -3.85861 & -0.95654 & 1.37627 \\ \mathrm{H} & -4.68224 & 0.56627 & 0.89641 \\ \mathrm{H} & -4.33828 & -0.70997 & -0.32117 \\ & & & \end{array}$

$\mathrm{E}(\mathrm{UB}+\mathrm{HF}-\mathrm{LYP})=-539.846959328$

$\mathrm{S} * * 2=0.5917$

1 imaginary frequency

$383 \mathrm{~K}, 1.00 \mathrm{~atm}$, freq's scaled by 1.0000

Zero-point correction $=0.216514$

Thermal correction to $\mathrm{U}=0.236455$

Thermal correction to $\mathrm{H}=0.237668$

Thermal correction to $\mathrm{G}=0.161291$

\section{TS ' -Ed-2d-x-trans}

UB3LYP / 6-31+G (d) :

$\begin{array}{lrrr}\mathrm{C} & -0.53033 & -1.46748 & 0.69069 \\ \mathrm{C} & -0.55108 & -0.06661 & 0.68070 \\ \mathrm{C} & 0.45286 & 0.77398 & 0.19454 \\ \mathrm{C} & -1.90151 & 0.56053 & 1.09996 \\ \mathrm{C} & -2.72084 & 0.79112 & -0.14084 \\ \mathrm{C} & -3.04964 & -0.24358 & -0.98878 \\ \mathrm{C} & -2.89006 & -1.60344 & -0.67003 \\ \mathrm{H} & -1.76210 & 1.49082 & 1.65616 \\ \mathrm{H} & -2.39519 & -0.14245 & 1.78522 \\ \mathrm{H} & -3.38019 & 0.00765 & -1.99760 \\ \mathrm{H} & -2.98696 & -1.95734 & 0.34669 \\ \mathrm{H} & -2.91750 & 1.81509 & -0.44879 \\ \mathrm{H} & -2.95745 & -2.36224 & -1.44491 \\ \mathrm{H} & 0.24301 & -2.03486 & 0.19360 \\ \mathrm{H} & -1.17232 & -2.01085 & 1.37399 \\ \mathrm{C} & 1.83233 & 0.34348 & -0.15397 \\ \mathrm{O} & 2.63458 & 1.09184 & -0.69624 \\ \mathrm{O} & 2.16820 & -0.92619 & 0.18849 \\ \mathrm{C} & 0.28114 & 2.26193 & -0.00667 \\ \mathrm{H} & 0.44562 & 2.52456 & -1.05875 \\ \mathrm{H} & -0.70917 & 2.61772 & 0.27624 \\ \mathrm{H} & 1.03351 & 2.82292 & 0.56155 \\ \mathrm{C} & 3.51331 & -1.31892 & -0.13318 \\ \mathrm{H} & 4.23256 & -0.67717 & 0.38307 \\ \mathrm{H} & 3.60094 & -2.35008 & 0.21211 \\ \mathrm{H} & 3.68586 & -1.25503 & -1.21103\end{array}$

$\mathrm{E}(\mathrm{UB}+\mathrm{HF}-\mathrm{LYP})=-539.845144856$

$\mathrm{S} * * 2=0.5854$

1 imaginary frequency

$383 \mathrm{~K}, 1.00 \mathrm{~atm}$, freq's scaled by 1.0000

Zero-point correction $=0.216408$

Thermal correction to $\mathrm{U}=0.236460$

Thermal correction to $\mathrm{H}=0.237673$

Thermal correction to $\mathrm{G}=0.160760$ 
TS ' -Ed-2d-n-cis

UB3LYP / 6-31+G (d) :

$\begin{array}{lrrr}\mathrm{C} & -2.89544 & -1.14569 & 1.19455 \\ \mathrm{C} & -3.68116 & -0.44304 & 0.25901 \\ \mathrm{C} & -3.21990 & 0.63480 & -0.45977 \\ \mathrm{C} & -1.82597 & 1.17129 & -0.31013 \\ \mathrm{C} & -0.67456 & 0.13477 & -0.36630 \\ \mathrm{C} & -0.98909 & -1.20419 & -0.64936 \\ \mathrm{C} & 0.61931 & 0.59671 & -0.08564 \\ \mathrm{H} & -1.65263 & 1.94618 & -1.06666 \\ \mathrm{H} & -1.74485 & 1.68305 & 0.66216 \\ \mathrm{H} & -4.66549 & -0.84341 & 0.01595 \\ \mathrm{H} & -2.06150 & -0.68555 & 1.70936 \\ \mathrm{H} & -3.87527 & 1.11234 & -1.18380 \\ \mathrm{H} & -3.23083 & -2.10177 & 1.58722 \\ \mathrm{H} & -0.22913 & -1.96948 & -0.57781 \\ \mathrm{H} & -1.91835 & -1.46135 & -1.13392 \\ \mathrm{C} & 1.79794 & -0.29979 & -0.16332 \\ \mathrm{O} & 1.84288 & -1.45322 & -0.56876 \\ \mathrm{O} & 2.92040 & 0.32317 & 0.30299 \\ \mathrm{C} & 4.12189 & -0.46177 & 0.27951 \\ \mathrm{H} & 4.36132 & -0.76802 & -0.74281 \\ \mathrm{H} & 4.90009 & 0.19054 & 0.67848 \\ \mathrm{H} & 4.01049 & -1.35428 & 0.90182 \\ \mathrm{C} & 0.91167 & 2.02902 & 0.30579 \\ \mathrm{H} & 0.07087 & 2.69666 & 0.11356 \\ \mathrm{H} & 1.16051 & 2.10834 & 1.37373 \\ \mathrm{H} & 1.78035 & 2.41452 & -0.23706\end{array}$

$\mathrm{E}(\mathrm{UB}+\mathrm{HF}-\mathrm{LYP})=-539.847782211$

$\mathrm{S} * * 2=0.6216$

1 imaginary frequency

$383 \mathrm{~K}, 1.00 \mathrm{~atm}$, freq's scaled by 1.0000

Zero-point correction $=0.216274$

Thermal correction to $U=0.236406$

Thermal correction to $\mathrm{H}=0.237619$

Thermal correction to $\mathrm{G}=0.160211$

\section{TS ' -Ed-2d-n-trans}

UB3LYP / 6-31+G (d) :

$\begin{array}{lrrr}\mathrm{C} & -2.73685 & -1.15023 & 1.26758 \\ \mathrm{C} & -3.51928 & -0.71020 & 0.18009 \\ \mathrm{C} & -3.13107 & 0.29719 & -0.67109 \\ \mathrm{C} & -1.82964 & 1.02930 & -0.51803 \\ \mathrm{C} & -0.56013 & 0.16114 & -0.32196 \\ \mathrm{C} & -0.68880 & -1.23441 & -0.39990 \\ \mathrm{C} & 0.61744 & 0.85501 & -0.01238 \\ \mathrm{H} & -1.68002 & 1.69155 & -1.37977 \\ \mathrm{H} & -1.89207 & 1.69757 & 0.35487 \\ \mathrm{H} & -4.42181 & -1.27221 & -0.06055 \\ \mathrm{H} & -2.02115 & -0.50419 & 1.75988 \\ \mathrm{H} & -3.77057 & 0.56296 & -1.50910 \\ \mathrm{H} & -2.98627 & -2.07127 & 1.78755 \\ \mathrm{H} & 0.13614 & -1.88118 & -0.13835 \\ \mathrm{H} & -1.52680 & -1.68463 & -0.90935 \\ \mathrm{C} & 1.96702 & 0.25311 & 0.12466 \\ \mathrm{O} & 2.91091 & 0.84292 & 0.63494 \\ \mathrm{O} & 2.11147 & -1.00177 & -0.37639 \\ \mathrm{C} & 3.42561 & -1.57125 & -0.25026 \\ \mathrm{H} & 3.71447 & -1.64470 & 0.80179 \\ \mathrm{H} & 3.35292 & -2.56190 & -0.70153 \\ \mathrm{H} & 4.16017 & -0.95932 & -0.78086 \\ \mathrm{C} & 0.61031 & 2.34917 & 0.23352 \\ \mathrm{H} & 0.04035 & 2.88510 & -0.53436 \\ \mathrm{H} & 0.15860 & 2.60289 & 1.20358 \\ \mathrm{H} & 1.62935 & 2.73716 & 0.24444\end{array}$

$\mathrm{E}(\mathrm{UB}+\mathrm{HF}-\mathrm{LYP})=-539.846052793$

$\mathrm{S} * * 2=0.6158$

1 imaginary frequency

$383 \mathrm{~K}, 1.00 \mathrm{~atm}$, freq's scaled by 1.0000

Zero-point correction $=0.216204$

Thermal correction to $\mathrm{U}=0.236357$

Thermal correction to $\mathrm{H}=0.237570$

Thermal correction to $\mathrm{G}=0.160231$ 
A reviewer suggested, and we agree, that it would be desirable to examine the cumulative effect of the various substituents present in 1a in order to explain how the Jung reactions proceed under much more mild conditions than the parent reaction. Full-scale computation of these large systems was not feasible due to wavefunction convergence problems encountered with singlet-state optimizations. Therefore, we roughly approximated the trend in activation energy by examining the trend in homolysis energy, which was in turn approximated by optimizing diradicals in the triplet state, and then performing a singlepoint electronic energy calculation on those structures in the singlet state. This method provided a reasonable approximation to singlet-state optimization, as can be seen by comparing the energies of $\mathbf{6}^{*}$ and $\mathbf{6} \mathbf{c}^{*}$ to their singlet-optimized counterparts, $\mathbf{6}$ and 6c. Table S1 summarizes these results; the effect appears highly additive, with the sum of $\Delta \Delta H\left(6^{*}-1\right)$ for the fullysubstituted system 1a/6a (-7.5) being very close to the sum of the sum of those for siloxy (-6.2), phenyl (-4.5), and estermethyl (+3.3) cases individually. Computational data and illustrations are shown on the following pages.

Table S1. Approximated homolysis enthalpies for variously-substituted derivatives of 3-vinylmethylenecyclobutane (UB3LYP/6-31+G(d)).

\begin{tabular}{lcc}
\hline substitution & $\Delta H\left(\mathbf{6}^{*-1}\right)$ & $\Delta \Delta H\left(\mathbf{6}^{*}-\mathbf{1}\right)$ \\
\hline none (parent) & 27.6 & 0.0 \\
siloxy (c) & 21.4 & -6.2 \\
phenyl & 23.1 & -4.5 \\
ester & 27.5 & -0.1 \\
ester-methyl $(\mathbf{d})$ & 30.9 & +3.3 \\
all $(\mathbf{a})$ & 20.1 & -7.5 \\
\hline
\end{tabular}

1

RB3LYP / 6-31+G(d) :

$\begin{array}{lrrr}\text { C } & -0.498928 & 0.310982 & 0.553194 \\ \mathrm{C} & 0.596993 & 1.142879 & -0.205680 \\ \mathrm{C} & 1.493396 & -0.083844 & -0.120325 \\ \mathrm{C} & 0.316495 & -0.981386 & 0.234561 \\ \mathrm{H} & 0.952547 & 2.067068 & 0.263621 \\ \mathrm{H} & 0.289594 & 1.363998 & -1.236538 \\ \mathrm{H} & 0.455423 & -1.708313 & 1.042291 \\ \mathrm{H} & -0.076916 & -1.505924 & -0.645914 \\ \mathrm{C} & -1.925139 & 0.452830 & 0.116674 \\ \mathrm{H} & -2.337283 & 1.459942 & 0.215295 \\ \mathrm{C} & -2.711544 & -0.517261 & -0.361885 \\ \mathrm{H} & -2.363660 & -1.541633 & -0.475923 \\ \mathrm{C} & 2.811450 & -0.267501 & -0.173891 \\ \mathrm{H} & -3.740314 & -0.320165 & -0.652511 \\ \mathrm{H} & -0.425666 & 0.536791 & 1.626234 \\ \mathrm{H} & 3.256977 & -1.246600 & -0.009426 \\ \mathrm{H} & 3.492961 & 0.554639 & -0.383020\end{array}$

$\mathrm{E}(\mathrm{RB}+\mathrm{HF}-\mathrm{LYP})=-272.700155$

$298 \mathrm{~K}, 1.00 \mathrm{~atm}$, freq's scaled by 1.0000

Zero-point correction $=0.148045$

Thermal correction to $U=0.155289$

Thermal correction to $\mathrm{H}=0.156233$

Thermal correction to $\mathrm{G}=0.116654$
6 *

UB3LYP/6-31+G(d) (triplet):

$\begin{array}{lrrr}\text { C } & 0.810763 & -0.774265 & -0.445400 \\ \mathrm{C} & -2.619882 & -0.635128 & 0.018495 \\ \mathrm{C} & -1.470698 & 0.139818 & 0.109946 \\ \mathrm{C} & -0.181094 & -0.487265 & 0.648185 \\ \mathrm{H} & -3.545849 & -0.221930 & -0.372753 \\ \mathrm{H} & -2.635472 & -1.673085 & 0.338050 \\ \mathrm{H} & -0.433551 & -1.425622 & 1.163626 \\ \mathrm{H} & 0.255861 & 0.172898 & 1.404845 \\ \mathrm{C} & 2.123279 & -0.309059 & -0.516734 \\ \mathrm{H} & 2.692292 & -0.625432 & -1.391818 \\ \mathrm{C} & 2.785716 & 0.502341 & 0.394321 \\ \mathrm{H} & 2.312535 & 0.875262 & 1.297768 \\ \mathrm{C} & -1.451774 & 1.476160 & -0.290619 \\ \mathrm{H} & 3.818149 & 0.793038 & 0.228438 \\ \mathrm{H} & 0.448317 & -1.399974 & -1.259471 \\ \mathrm{H} & -0.548904 & 2.075857 & -0.225139 \\ \mathrm{H} & -2.341237 & 1.953379 & -0.692711\end{array}$

$\mathrm{E}(\mathrm{UB}+\mathrm{HF}-\mathrm{LYP}) \quad($ singlet $)=-272.652227$

$298 \mathrm{~K}, 1.00 \mathrm{~atm}$, freq's scaled by 1.0000

Zero-point correction $=0.143046$

Thermal correction to $\mathrm{U}=0.151389$

Thermal correction to $\mathrm{H}=0.152333$

Thermal correction to $\mathrm{G}=0.107979$ 
$1 c$

RB3LYP / 6-31+G (d) :

$\begin{array}{lrrr}\mathrm{C} & 0.838703 & 0.323329 & -0.198758 \\ \mathrm{C} & 1.305758 & -0.773682 & 0.837629 \\ \mathrm{C} & 2.532874 & -0.990215 & -0.032744 \\ \mathrm{C} & 1.903791 & -0.234230 & -1.190324 \\ \mathrm{H} & 0.633981 & -1.640794 & 0.826515 \\ \mathrm{H} & 1.463808 & -0.450877 & 1.872573 \\ \mathrm{H} & 1.409804 & -0.902050 & -1.906240 \\ \mathrm{H} & 2.521358 & 0.477784 & -1.747007 \\ \mathrm{C} & 1.073907 & 1.722706 & 0.319727 \\ \mathrm{H} & 0.301524 & 2.064655 & 1.009487 \\ \mathrm{C} & 2.098516 & 2.535114 & 0.048181 \\ \mathrm{H} & 2.908190 & 2.259378 & -0.621519 \\ \mathrm{O} & -0.482723 & 0.232026 & -0.702910 \\ \mathrm{Si} & -1.970078 & -0.151867 & -0.019995 \\ \mathrm{C} & -3.230930 & 0.688562 & -1.135402 \\ \mathrm{H} & -3.130860 & 0.339359 & -2.170374 \\ \mathrm{H} & -4.257732 & 0.479779 & -0.808099 \\ \mathrm{H} & -3.090903 & 1.776291 & -1.138190 \\ \mathrm{C} & -2.112370 & 0.484602 & 1.755892 \\ \mathrm{H} & -1.324553 & 0.082075 & 2.404906 \\ \mathrm{H} & -2.059038 & 1.579399 & 1.801669 \\ \mathrm{H} & -3.075907 & 0.185068 & 2.189670 \\ \mathrm{C} & -2.241979 & -2.023403 & -0.041791 \\ \mathrm{H} & -2.060059 & -2.432901 & -1.043360 \\ \mathrm{H} & -1.585569 & -2.553542 & 0.659183 \\ \mathrm{H} & -3.277397 & -2.266340 & 0.232589 \\ \mathrm{C} & 3.733065 & -1.523965 & 0.184786 \\ \mathrm{H} & 2.163578 & 3.520824 & 0.501954 \\ \mathrm{H} & 3.991267 & -1.976420 & 1.140022 \\ \mathrm{H} & 4.503377 & -1.524670 & -0.583751\end{array}$

$\mathrm{E}(\mathrm{RB}+\mathrm{HF}-\mathrm{LYP})=-756.634400$

$298 \mathrm{~K}, 1.00 \mathrm{~atm}$, freq's scaled by 1.0000

zero-point correction $=0.253571$

Thermal correction to $\mathrm{U}=0.269716$

Thermal correction to $\mathrm{H}=0.270660$

Thermal correction to $\mathrm{G}=0.209316$

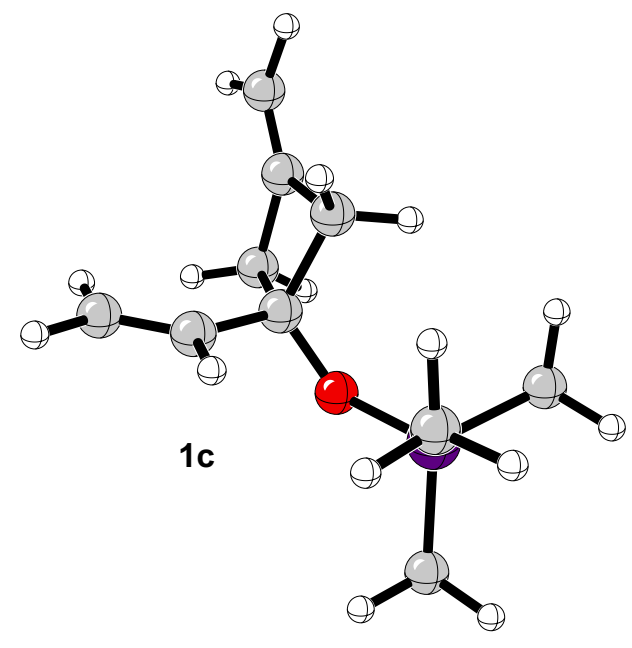

$6 c *$

UB3LYP/6-31+G(d) (triplet) :

$\begin{array}{lrrr}\text { C } & 0.681710 & 0.695040 & -0.548108 \\ \mathrm{C} & 2.441033 & -2.314830 & -0.342189 \\ \mathrm{C} & 2.529201 & -0.965995 & -0.023902 \\ \mathrm{C} & 1.974419 & 0.073870 & -1.003355 \\ \mathrm{H} & 2.825524 & -3.075776 & 0.332277 \\ \mathrm{H} & 1.986346 & -2.652711 & -1.268262 \\ \mathrm{H} & 1.798158 & -0.406535 & -1.974487 \\ \mathrm{H} & 2.720628 & 0.857713 & -1.164793 \\ \mathrm{C} & 0.530866 & 2.010462 & -0.102906 \\ \mathrm{H} & -0.484033 & 2.312589 & 0.152216 \\ \mathrm{C} & 1.518492 & 2.973660 & 0.043873 \\ \mathrm{H} & 2.564039 & 2.785150 & -0.178421 \\ \mathrm{O} & -0.368866 & -0.164902 & -0.637260 \\ \mathrm{Si} & -1.898322 & -0.270634 & 0.092344 \\ \mathrm{C} & -2.472212 & -2.001211 & -0.363428 \\ \mathrm{H} & -1.782936 & -2.758880 & 0.027859 \\ \mathrm{H} & -3.470335 & -2.209689 & 0.042927 \\ \mathrm{H} & -2.521295 & -2.124929 & -1.452115 \\ \mathrm{C} & -3.067337 & 1.020396 & -0.636175 \\ \mathrm{H} & -2.800031 & 2.044882 & -0.351789 \\ \mathrm{H} & -3.073032 & 0.969740 & -1.732089 \\ \mathrm{H} & -4.094485 & 0.841187 & -0.291087 \\ \mathrm{C} & -1.737428 & -0.067480 & 1.959582 \\ \mathrm{H} & -2.708724 & -0.231298 & 2.445130 \\ \mathrm{H} & -1.030106 & -0.795687 & 2.374521 \\ \mathrm{H} & -1.390184 & 0.932638 & 2.243471 \\ \mathrm{C} & 3.109314 & -0.529817 & 1.167557 \\ \mathrm{H} & 3.172804 & 0.524499 & 1.418699 \\ \mathrm{H} & 3.503404 & -1.240273 & 1.889278 \\ \mathrm{H} & 1.263348 & 3.970913 & 0.386234\end{array}$

$\mathrm{E}(\mathrm{UB}+\mathrm{HF}-\mathrm{LYP}) \quad($ singlet $)=-756.597225$ $298 \mathrm{~K}, 1.00 \mathrm{~atm}$, freq's scaled by 1.0000 Zero-point correction $=0.249467$ Thermal correction to $\mathrm{U}=0.266716$ Thermal correction to $\mathrm{H}=0.267660$ Thermal correction to $\mathrm{G}=0.201707$

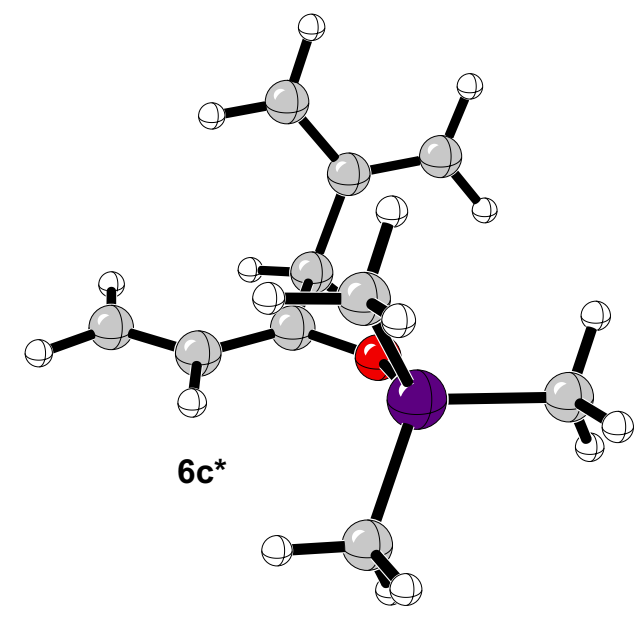




\section{1-phenyl}

RB3LYP / 6-31+G (d) :

$\begin{array}{lrrr}\text { C } & -2.114752 & -0.455857 & 0.656876 \\ \mathrm{C} & -3.098913 & -1.093172 & -0.390784 \\ \mathrm{C} & -3.928825 & 0.177628 & -0.285018 \\ \mathrm{C} & -2.788106 & 0.927024 & 0.388765 \\ \mathrm{H} & -3.581423 & -2.040830 & -0.126489 \\ \mathrm{H} & -2.621790 & -1.199260 & -1.374212 \\ \mathrm{H} & -3.030097 & 1.551448 & 1.255788 \\ \mathrm{H} & -2.218012 & 1.530446 & -0.329677 \\ \mathrm{C} & -0.644515 & -0.659961 & 0.464708 \\ \mathrm{H} & -0.326464 & -1.703720 & 0.496147 \\ \mathrm{C} & 0.269211 & 0.307437 & 0.276342 \\ \mathrm{H} & -0.072533 & 1.342571 & 0.264361 \\ \mathrm{C} & 1.722132 & 0.147436 & 0.090528 \\ \mathrm{C} & 2.366345 & -1.104095 & 0.026607 \\ \mathrm{C} & 2.519039 & 1.301006 & -0.032005 \\ \mathrm{C} & 3.745880 & -1.192716 & -0.149115 \\ \mathrm{H} & 1.785485 & -2.018311 & 0.111524 \\ \mathrm{C} & 3.901568 & 1.215100 & -0.207718 \\ \mathrm{H} & 2.043750 & 2.278922 & 0.012824 \\ \mathrm{C} & 4.523158 & -0.034067 & -0.266894 \\ \mathrm{H} & 4.218696 & -2.170793 & -0.196153 \\ \mathrm{H} & 4.491105 & 2.124117 & -0.298515 \\ \mathrm{H} & 5.598881 & -0.107265 & -0.404178 \\ \mathrm{C} & -5.203688 & 0.469958 & -0.536044 \\ \mathrm{H} & -5.617333 & 1.451235 & -0.312079 \\ \mathrm{H} & -5.880221 & -0.261283 & -0.973812 \\ \mathrm{H} & -2.401246 & -0.811585 & 1.656982\end{array}$

$E(R B+H F-L Y P)=-503.767535$

$298 \mathrm{~K}, 1.00 \mathrm{~atm}$, freq's scaled by 1.0000

Zero-point correction $=0.229543$

Thermal correction to $\mathrm{U}=0.241337$

Thermal correction to $\mathrm{H}=0.242281$

Thermal correction to $\mathrm{G}=0.189595$

\section{6-phenyl *}

UB3LYP/6-31+G(d) (triplet):

$\begin{array}{lrrr}\mathrm{C} & -1.901938 & 1.074692 & -0.335280 \\ \mathrm{C} & -5.230241 & -0.032289 & -0.229884 \\ \mathrm{C} & -3.932650 & -0.339883 & 0.157320 \\ \mathrm{C} & -2.773767 & -0.059077 & -0.803854 \\ \mathrm{H} & -6.071602 & -0.212909 & 0.433980 \\ \mathrm{H} & -5.449432 & 0.392287 & -1.205253 \\ \mathrm{H} & -3.189190 & 0.191111 & -1.790943 \\ \mathrm{H} & -2.187099 & -0.973730 & -0.939783 \\ \mathrm{C} & -0.538111 & 1.053208 & -0.137387 \\ \mathrm{H} & -0.098249 & 1.989067 & 0.204143 \\ \mathrm{C} & 0.331779 & -0.037826 & -0.333610 \\ \mathrm{H} & -0.095229 & -0.980507 & -0.669512 \\ \mathrm{C} & 1.759179 & -0.045567 & -0.137504 \\ \mathrm{C} & 2.504712 & 1.083491 & 0.292280 \\ \mathrm{C} & 2.480939 & -1.242583 & -0.384223 \\ \mathrm{C} & 3.883266 & 1.011213 & 0.462996 \\ \mathrm{H} & 1.998302 & 2.022681 & 0.493241 \\ \mathrm{C} & 3.859719 & -1.310095 & -0.212508 \\ \mathrm{H} & 1.935672 & -2.124004 & -0.715454 \\ \mathrm{C} & 4.573574 & -0.182822 & 0.213102 \\ \mathrm{H} & 4.427935 & 1.892405 & 0.793608 \\ \mathrm{H} & 4.382169 & -2.242877 & -0.410480 \\ \mathrm{H} & 5.650720 & -0.232542 & 0.347992 \\ \mathrm{C} & -3.650165 & -0.891190 & 1.407802 \\ \mathrm{H} & -2.632721 & -1.117158 & 1.712437 \\ \mathrm{H} & -4.443628 & -1.098888 & 2.120318 \\ \mathrm{H} & -2.425421 & 2.007427 & -0.129788\end{array}$

$\mathrm{E}(\mathrm{UB}+\mathrm{HF}-\mathrm{LYP}) \quad($ singlet $)=-503.727100$ $298 \mathrm{~K}, 1.00 \mathrm{~atm}$, freq's scaled by 1.0000 Zero-point correction $=0.225012$ Thermal correction to $\mathrm{U}=0.237746$ Thermal correction to $\mathrm{H}=0.238690$ Thermal correction to $\mathrm{G}=0.181980$

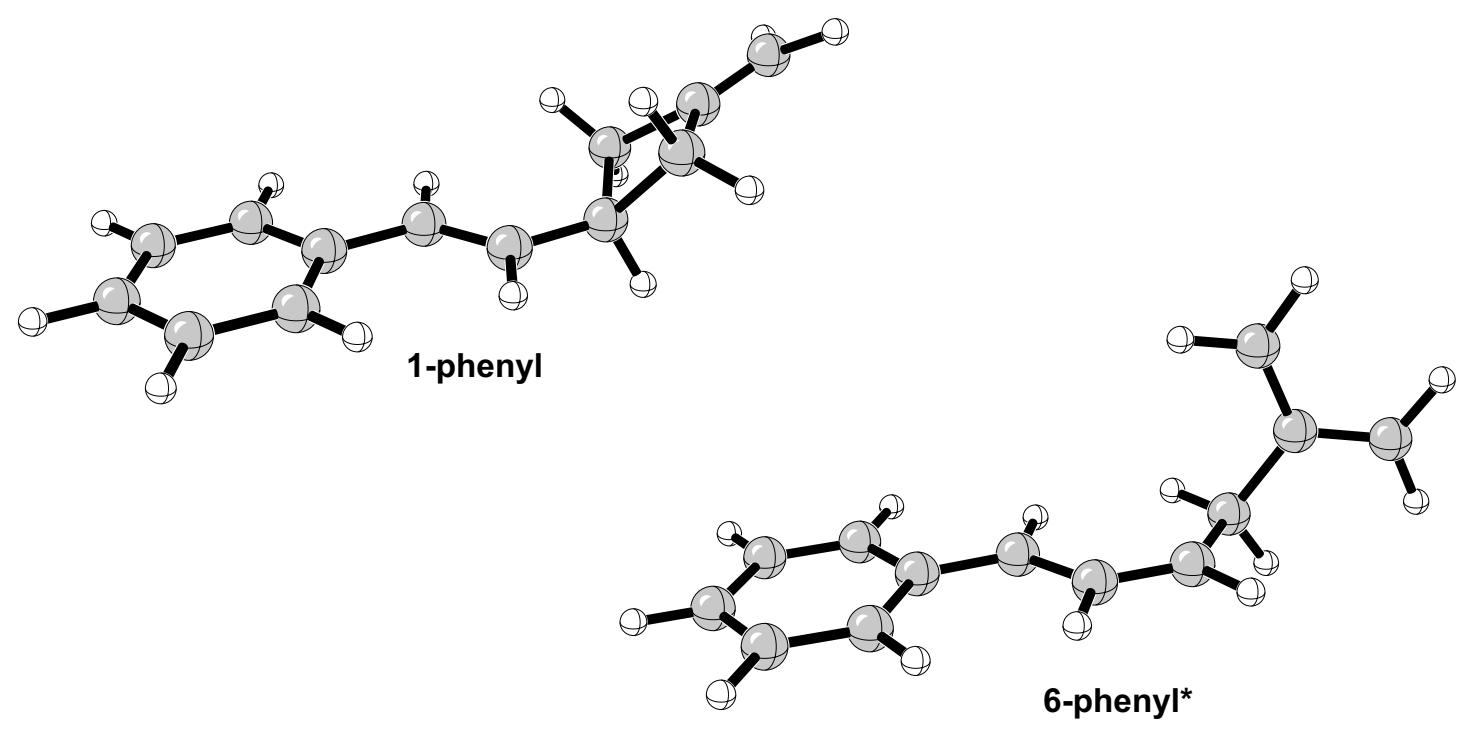




\section{1-ester}

RB3LYP / 6-31+G (d) :

$\begin{array}{lrrr}\mathrm{C} & 3.821223 & 1.360165 & -0.485705 \\ \mathrm{C} & 3.545712 & 0.186361 & 0.092345 \\ \mathrm{C} & 2.208299 & -0.269212 & 0.591544 \\ \mathrm{C} & 0.907245 & 0.511491 & 0.227636 \\ \mathrm{C} & 0.244714 & -0.820972 & -0.066776 \\ \mathrm{C} & -1.014040 & -1.271234 & -0.168241 \\ \mathrm{C} & -2.244677 & -0.462373 & -0.101573 \\ \mathrm{O} & -2.016210 & 0.869572 & 0.044061 \\ \mathrm{C} & -3.190718 & 1.696715 & 0.108965 \\ \mathrm{C} & 1.576343 & -1.549512 & -0.063539 \\ \mathrm{H} & -1.202334 & -2.331527 & -0.317676 \\ \mathrm{O} & -3.367449 & -0.933038 & -0.175023 \\ \mathrm{H} & 1.632879 & -2.492861 & 0.491060 \\ \mathrm{H} & 1.956007 & -1.715262 & -1.080611 \\ \mathrm{H} & 0.447625 & 1.135018 & 0.998931 \\ \mathrm{H} & 1.014696 & 1.116355 & -0.681856 \\ \mathrm{H} & 4.357132 & -0.531204 & 0.234413 \\ \mathrm{H} & 3.060641 & 2.121121 & -0.646109 \\ \mathrm{H} & -3.778311 & 1.599173 & -0.808029 \\ \mathrm{H} & -3.811504 & 1.415037 & 0.964004 \\ \mathrm{H} & -2.820049 & 2.716407 & 0.221681 \\ \mathrm{H} & 4.827627 & 1.605588 & -0.814853 \\ \mathrm{H} & 2.260252 & -0.418687 & 1.678805\end{array}$

$\mathrm{E}(\mathrm{RB}+\mathrm{HF}-\mathrm{LYP})=-500.590613$

$298 \mathrm{~K}, 1.00 \mathrm{~atm}$, freq's scaled by 1.0000

Zero-point correction $=0.191631$

Thermal correction to $\mathrm{U}=0.203255$

Thermal correction to $\mathrm{H}=0.204199$

Thermal correction to $\mathrm{G}=0.153011$ 6-ester*

UB3LYP/6-31+G(d) (triplet) :

\begin{tabular}{|c|c|c|c|}
\hline $\mathrm{C}$ & -1.215956 & 2.788115 & -0.144824 \\
\hline $\mathrm{C}$ & -0.682829 & 1.539594 & 0.142820 \\
\hline $\mathrm{C}$ & 0.666068 & 1.304448 & -0.175890 \\
\hline $\mathrm{C}$ & 1.452789 & 0.107670 & 0.117924 \\
\hline $\mathrm{O}$ & 2.700848 & 0.228321 & -0.416061 \\
\hline $\mathrm{C}$ & 3.586774 & -0.879217 & -0.190162 \\
\hline $\mathrm{C}$ & -1.596493 & 0.480976 & 0.759444 \\
\hline $\mathrm{C}$ & -2.462864 & -0.168034 & -0.290724 \\
\hline $\mathrm{C}$ & -2.542071 & -1.531084 & -0.574561 \\
\hline $\mathrm{C}$ & -1.812333 & -2.560383 & 0.002639 \\
\hline $\mathrm{H}$ & 1.216373 & 2.098666 & -0.672871 \\
\hline $\mathrm{O}$ & 1.108989 & -0.878745 & 0.756191 \\
\hline $\mathrm{H}$ & -0.620325 & 3.564170 & -0.617893 \\
\hline $\mathrm{H}$ & -2.246175 & 3.032582 & 0.09825 \\
\hline $\mathrm{H}$ & -2.244344 & 0.983122 & 1.494162 \\
\hline $\mathrm{H}$ & -1.005040 & -0.260254 & 1.294563 \\
\hline $\mathrm{H}$ & -3.266455 & -1.810870 & -1.341019 \\
\hline $\mathrm{H}$ & -1.033710 & -2.380355 & 0.73639 \\
\hline $\mathrm{H}$ & 3.747043 & -1.029402 & 0.88138 \\
\hline $\mathrm{H}$ & 3.173141 & -1.794829 & -0.62246 \\
\hline $\mathrm{H}$ & 4.520202 & -0.607787 & -0.68461 \\
\hline $\mathrm{H}$ & -1.982045 & -3.589340 & -0.29958 \\
\hline $\mathrm{H}$ & -3.095877 & 0.505177 & -0.86736 \\
\hline
\end{tabular}

$\mathrm{E}(\mathrm{UB}+\mathrm{HF}-\mathrm{LYP}) \quad($ singlet $)=-500.543488$ $298 \mathrm{~K}, 1.00 \mathrm{~atm}$, freq's scaled by 1.0000 Zero-point correction $=0.187522$ Thermal correction to $\mathrm{U}=0.199953$ Thermal correction to $\mathrm{H}=0.200897$ Thermal correction to $\mathrm{G}=0.146723$

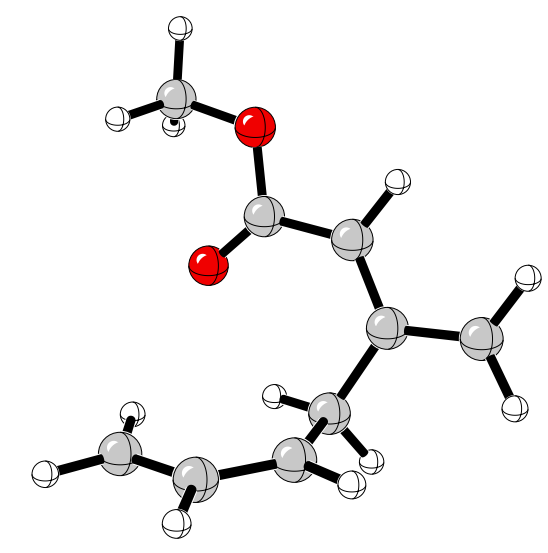

6-ester* 
$1 d$

RB3LYP / 6-31+G (d) :

$\begin{array}{lrrr}\mathrm{C} & -2.314904 & 0.160485 & 0.634845 \\ \mathrm{C} & -1.585083 & 1.437715 & 0.086809 \\ \mathrm{C} & -0.299631 & 0.625921 & 0.045316 \\ \mathrm{C} & -1.045837 & -0.674981 & 0.287003 \\ \mathrm{H} & -1.595962 & 2.331049 & 0.721905 \\ \mathrm{H} & -1.934827 & 1.720110 & -0.915583 \\ \mathrm{H} & -0.649631 & -1.335575 & 1.062968 \\ \mathrm{H} & -1.150755 & -1.262559 & -0.633694 \\ \mathrm{C} & -3.649152 & -0.184705 & 0.045838 \\ \mathrm{H} & -4.424753 & 0.570186 & 0.195686 \\ \mathrm{C} & -3.963890 & -1.301831 & -0.618336 \\ \mathrm{H} & -3.242143 & -2.096520 & -0.793566 \\ \mathrm{C} & 0.988112 & 0.996271 & -0.076710 \\ \mathrm{C} & 1.405607 & 2.436887 & -0.252979 \\ \mathrm{H} & 2.083915 & 2.751583 & 0.548804 \\ \mathrm{H} & 1.952450 & 2.575299 & -1.193401 \\ \mathrm{H} & 0.537079 & 3.101503 & -0.258175 \\ \mathrm{C} & 2.111723 & 0.025527 & -0.049850 \\ \mathrm{O} & 3.286450 & 0.351541 & -0.103498 \\ \mathrm{O} & 1.723270 & -1.273777 & 0.030504 \\ \mathrm{C} & 2.788370 & -2.239091 & 0.055533 \\ \mathrm{H} & 3.387022 & -2.172871 & -0.857170 \\ \mathrm{H} & 3.435996 & -2.074114 & 0.921087 \\ \mathrm{H} & 2.295787 & -3.210108 & 0.122649 \\ \mathrm{H} & -4.965778 & -1.464245 & -1.007449 \\ \mathrm{H} & -2.418050 & 0.250955 & 1.725073\end{array}$

$\mathrm{E}(\mathrm{RB}+\mathrm{HF}-\mathrm{LYP})=-539.908535$

$298 \mathrm{~K}, 1.00 \mathrm{~atm}$, freq's scaled by 1.0000

Zero-point correction $=0.219433$

Thermal correction to $\mathrm{U}=0.232723$

Thermal correction to $\mathrm{H}=0.233667$

Thermal correction to $\mathrm{G}=0.178802$
$6 d *$

UB3LYP/6-31+G(d) (triplet) :

$\begin{array}{lrrr}\mathrm{C} & -2.347093 & 0.082734 & -0.540263 \\ \mathrm{C} & -0.777630 & 2.903416 & 0.139459 \\ \mathrm{C} & -0.498621 & 1.561583 & 0.293747 \\ \mathrm{C} & -1.669116 & 0.650937 & 0.680309 \\ \mathrm{H} & -0.035766 & 3.630840 & -0.169969 \\ \mathrm{H} & -1.775220 & 3.285389 & 0.336933 \\ \mathrm{H} & -2.397005 & 1.266063 & 1.230605 \\ \mathrm{H} & -1.340075 & -0.137143 & 1.354459 \\ \mathrm{C} & -2.655057 & -1.256888 & -0.777439 \\ \mathrm{H} & -3.198896 & -1.459038 & -1.701606 \\ \mathrm{C} & -2.357408 & -2.349815 & 0.023986 \\ \mathrm{H} & -1.782087 & -2.258891 & 0.939339 \\ \mathrm{C} & 0.811714 & 1.065960 & 0.017292 \\ \mathrm{C} & 1.871512 & 1.981653 & -0.548817 \\ \mathrm{H} & 2.074317 & 2.818576 & 0.133465 \\ \mathrm{H} & 2.807676 & 1.450448 & -0.716180 \\ \mathrm{H} & 1.554168 & 2.416468 & -1.505912 \\ \mathrm{C} & 1.186008 & -0.335480 & 0.304929 \\ \mathrm{O} & 0.641166 & -1.082336 & 1.106610 \\ \mathrm{O} & 2.269557 & -0.737500 & -0.414057 \\ \mathrm{C} & 2.710076 & -2.084393 & -0.176311 \\ \mathrm{H} & 2.995591 & -2.214872 & 0.871299 \\ \mathrm{H} & 1.916651 & -2.795192 & -0.423216 \\ \mathrm{H} & 3.570837 & -2.225039 & -0.831297 \\ \mathrm{H} & -2.673978 & -3.347110 & -0.266109 \\ \mathrm{H} & -2.648309 & 0.809950 & -1.293586\end{array}$

$\mathrm{E}(\mathrm{UB}+\mathrm{HF}-\mathrm{LYP}) \quad(\mathrm{singlet})=-539.856291$ $298 \mathrm{~K}, 1.00 \mathrm{~atm}$, freq's scaled by 1.0000 Zero-point correction $=0.215640$ Thermal correction to $U=0.229666$ Thermal correction to $\mathrm{H}=0.230610$ Thermal correction to $\mathrm{G}=0.172600$

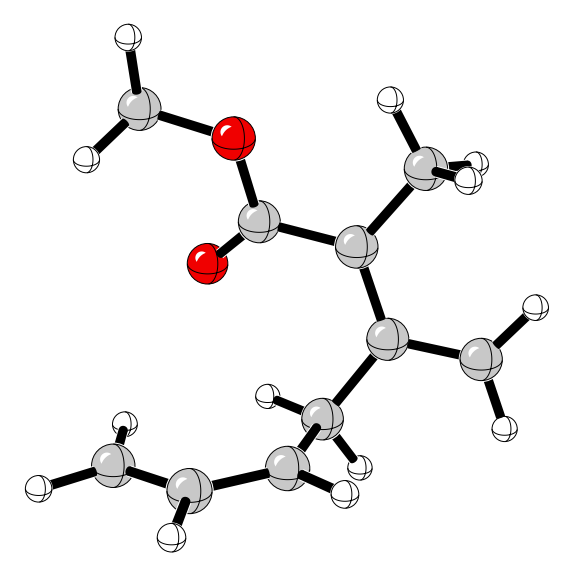

$6 d^{*}$ 
$1 a$

RB3LYP / 6-31+G (d) :

\begin{tabular}{|c|c|c|c|}
\hline C & -0.203568 & 0.890519 & -0.141362 \\
\hline C & -1.100133 & 0.959600 & 1.154873 \\
\hline C & -2.044957 & -0.031617 & 0.497803 \\
\hline C & -1.368465 & 0.137155 & -0.848008 \\
\hline $\mathrm{H}$ & -1.558743 & 1.948128 & 1.279813 \\
\hline $\mathrm{H}$ & -0.629178 & 0.661640 & 2.098667 \\
\hline $\mathrm{H}$ & -1.913195 & 0.829269 & -1.501144 \\
\hline $\mathrm{H}$ & -1.146005 & -0.762751 & -1.424307 \\
\hline $\mathrm{C}$ & 1.071007 & 0.117512 & 0.093739 \\
\hline $\mathrm{H}$ & 1.797286 & 0.661342 & 0.696611 \\
\hline C & 1.368170 & -1.115730 & -0.345121 \\
\hline $\mathrm{H}$ & 0.640337 & -1.646297 & -0.956153 \\
\hline $\mathrm{C}$ & 2.613442 & -1.867110 & -0.104996 \\
\hline C & 3.635722 & -1.425151 & 0.757535 \\
\hline $\mathrm{C}$ & 2.799521 & -3.093653 & -0.769219 \\
\hline $\mathrm{C}$ & 4.796378 & -2.175127 & 0.936716 \\
\hline $\mathrm{H}$ & 3.521681 & -0.491534 & 1.301258 \\
\hline C & 3.961564 & -3.846925 & -0.591681 \\
\hline $\mathrm{H}$ & 2.020915 & -3.457234 & -1.436770 \\
\hline C & 4.967702 & -3.390003 & 0.262261 \\
\hline $\mathrm{H}$ & 5.570356 & -1.813486 & 1.609328 \\
\hline $\mathrm{H}$ & 4.079025 & -4.789836 & -1.119922 \\
\hline $\mathrm{H}$ & 5.873512 & -3.973257 & 0.405894 \\
\hline 0 & 0.092472 & 2.123565 & -0.778227 \\
\hline $\mathrm{Si}$ & 0.697920 & 3.612581 & -0.285625 \\
\hline $\mathrm{C}$ & 1.230047 & 4.437805 & -1.889807 \\
\hline $\mathrm{H}$ & 0.386791 & 4.520724 & -2.586277 \\
\hline $\mathrm{H}$ & 1.618145 & 5.448715 & -1.709916 \\
\hline $\mathrm{H}$ & 2.015721 & 3.857136 & -2.387959 \\
\hline C & 2.166943 & 3.442492 & 0.894826 \\
\hline $\mathrm{H}$ & 1.895025 & 2.938438 & 1.831168 \\
\hline $\mathrm{H}$ & 2.995223 & 2.887385 & 0.437788 \\
\hline $\mathrm{H}$ & 2.546354 & 4.437624 & 1.163887 \\
\hline $\mathrm{C}$ & -0.648192 & 4.640539 & 0.557385 \\
\hline $\mathrm{H}$ & -1.560448 & 4.674755 & -0.051226 \\
\hline $\mathrm{H}$ & -0.918689 & 4.252027 & 1.546993 \\
\hline $\mathrm{H}$ & -0.305638 & 5.674694 & 0.697777 \\
\hline $\mathrm{C}$ & -3.034586 & -0.790960 & 1.000317 \\
\hline $\mathrm{C}$ & -3.432770 & -0.749375 & 2.456350 \\
\hline $\mathrm{H}$ & -4.479363 & -0.441687 & 2.569487 \\
\hline $\mathrm{H}$ & -3.350109 & -1.741372 & 2.915529 \\
\hline $\mathrm{H}$ & -2.805336 & -0.050640 & 3.016834 \\
\hline C & -3.824430 & -1.736639 & 0.170520 \\
\hline $\mathrm{O}$ & -4.687516 & -2.471340 & 0.622224 \\
\hline $\mathrm{O}$ & -3.513187 & -1.711296 & -1.151398 \\
\hline C & -4.265232 & -2.611262 & -1.983115 \\
\hline $\mathrm{H}$ & -4.108268 & -3.646828 & -1.668765 \\
\hline $\mathrm{H}$ & -5.332552 & -2.379309 & -1.930681 \\
\hline $\mathrm{H}$ & -3.886865 & -2.453637 & -2.994049 \\
\hline
\end{tabular}

$\mathrm{E}(\mathrm{RB}+\mathrm{HF}-\mathrm{LYP})=-1254.910185$

$298 \mathrm{~K}, 1.00 \mathrm{~atm}$, freq's scaled by 1.0000 Zero-point correction $=0.406484$ Thermal correction to $\mathrm{U}=0.432374$ Thermal correction to $\mathrm{H}=0.433318$ Thermal correction to $\mathrm{G}=0.347961$
$6 a *$

UB3LYP/6-31+G(d) (triplet):

\begin{tabular}{|c|c|c|c|}
\hline C & 0.983118 & -0.414568 & -0.851852 \\
\hline C & 3.340778 & 1.865949 & -0.821958 \\
\hline C & 1.964278 & 1.920181 & -0.773441 \\
\hline C & 1.202371 & 0.878082 & -1.602201 \\
\hline $\mathrm{H}$ & 3.976961 & 2.540953 & -0.260326 \\
\hline $\mathrm{H}$ & 3.840036 & 1.134528 & -1.448586 \\
\hline $\mathrm{H}$ & 1.808474 & 0.642809 & -2.487294 \\
\hline $\mathrm{H}$ & 0.255870 & 1.281178 & -1.951234 \\
\hline $\mathrm{C}$ & -0.240314 & -0.950257 & -0.490478 \\
\hline $\mathrm{H}$ & -0.202783 & -1.920592 & -0.000750 \\
\hline $\mathrm{C}$ & -1.508028 & -0.381853 & -0.703217 \\
\hline $\mathrm{H}$ & -1.563576 & 0.596012 & -1.172100 \\
\hline C & -2.773042 & -0.967988 & -0.340445 \\
\hline C & -2.916047 & -2.223611 & 0.307443 \\
\hline $\mathrm{C}$ & -3.966857 & -0.259031 & -0.640412 \\
\hline $\mathrm{C}$ & -4.171049 & -2.730013 & 0.630374 \\
\hline $\mathrm{H}$ & -2.035205 & -2.807560 & 0.558804 \\
\hline C & -5.219389 & -0.769312 & -0.315028 \\
\hline $\mathrm{H}$ & -3.888589 & 0.704179 & -1.140243 \\
\hline $\mathrm{C}$ & -5.334492 & -2.010663 & 0.324614 \\
\hline $\mathrm{H}$ & -4.246413 & -3.695389 & 1.125923 \\
\hline $\mathrm{H}$ & -6.112450 & -0.199649 & -0.561689 \\
\hline $\mathrm{H}$ & -6.311980 & -2.411613 & 0.579146 \\
\hline 0 & 2.155423 & -1.060298 & -0.597294 \\
\hline $\mathrm{Si}$ & 2.615884 & -2.401385 & 0.335948 \\
\hline $\mathrm{C}$ & 4.492829 & -2.375469 & 0.231149 \\
\hline $\mathrm{H}$ & 4.898149 & -1.442928 & 0.641839 \\
\hline $\mathrm{H}$ & 4.930337 & -3.209166 & 0.795437 \\
\hline $\mathrm{H}$ & 4.833466 & -2.459877 & -0.807929 \\
\hline $\mathrm{C}$ & 1.937118 & -3.991304 & -0.420090 \\
\hline $\mathrm{H}$ & 0.843848 & -4.053060 & -0.374005 \\
\hline $\mathrm{H}$ & 2.229556 & -4.078348 & -1.473870 \\
\hline $\mathrm{H}$ & 2.339245 & -4.865855 & 0.108685 \\
\hline $\mathrm{C}$ & 2.043265 & -2.185110 & 2.120194 \\
\hline $\mathrm{H}$ & 2.453627 & -2.986433 & 2.749096 \\
\hline $\mathrm{H}$ & 2.394127 & -1.229697 & 2.529482 \\
\hline $\mathrm{H}$ & 0.952601 & -2.209541 & 2.224479 \\
\hline $\mathrm{C}$ & 1.315495 & 2.876981 & 0.066008 \\
\hline $\mathrm{C}$ & 2.123242 & 3.711665 & 1.032805 \\
\hline $\mathrm{H}$ & 2.752926 & 4.437737 & 0.497364 \\
\hline $\mathrm{H}$ & 1.476571 & 4.270177 & 1.708322 \\
\hline $\mathrm{H}$ & 2.792951 & 3.088370 & 1.636973 \\
\hline $\mathrm{C}$ & -0.135056 & 3.143376 & -0.029006 \\
\hline 0 & -0.858140 & 2.888940 & -0.984957 \\
\hline 0 & -0.623523 & 3.764488 & 1.078280 \\
\hline C & -2.026174 & 4.075746 & 1.053503 \\
\hline $\mathrm{H}$ & -2.257141 & 4.737192 & 0.213857 \\
\hline $\mathrm{H}$ & -2.618832 & 3.161069 & 0.965995 \\
\hline $\mathrm{H}$ & -2.226507 & 4.573047 & 2.003336 \\
\hline
\end{tabular}

$\mathrm{E}(\mathrm{UB}+\mathrm{HF}-\mathrm{LYP}) \quad($ singlet $)=-1254.876120$ $298 \mathrm{~K}, 1.00 \mathrm{~atm}$, freq's scaled by 1.0000 Zero-point correction $=0.403629$ Thermal correction to $U=0.430318$ Thermal correction to $\mathrm{H}=0.431263$ Thermal correction to $\mathrm{G}=0.342517$ 

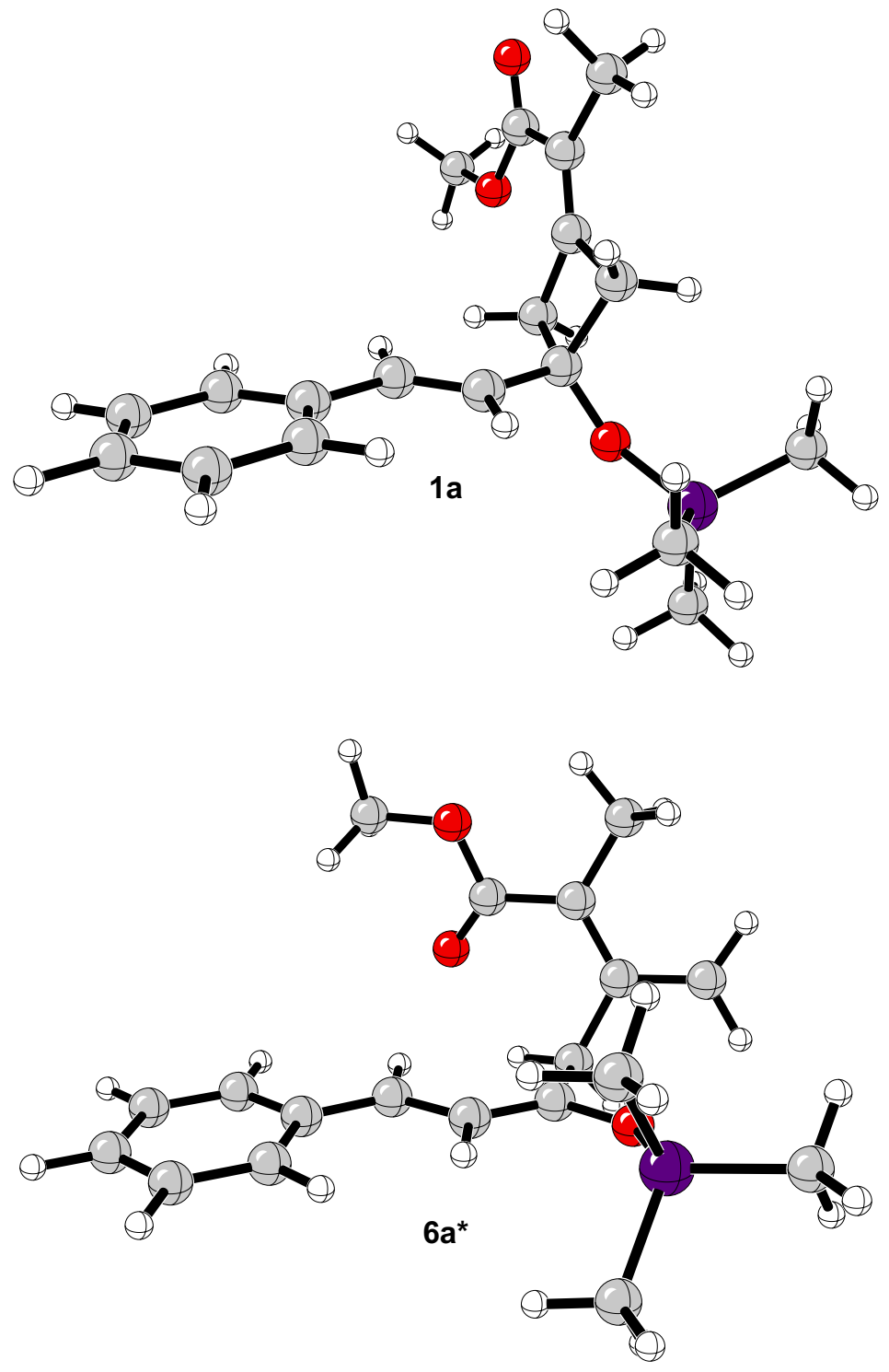
Table S2. Raw data for potential energy surface plot in Figure 2. Dihedral angles are listed in degrees. UB3LYP/6-31+G(d) electronic energies from constrained optimizations are listed in a.u. and in $\mathrm{kcal} / \mathrm{mol}$ relative to 1 . Table continues on following pages.

\begin{tabular}{|c|c|c|c|c|c|c|c|c|c|c|c|}
\hline $\mathrm{D}(3,4,5,6)$ & $\mathrm{D}(2,3,4,5)$ & E-ele (a.u.) & $\begin{array}{c}\text { E-ele-rel } \\
(\mathrm{kcal} / \mathrm{mol})\end{array}$ & $\mathrm{D}(3,4,5,6)$ & $\mathrm{D}(2,3,4,5)$ & E-ele (a.u.) & $\begin{array}{c}\text { E-ele-rel } \\
(\mathrm{kcal} / \mathrm{mol})\end{array}$ & $\mathrm{D}(3,4,5,6)$ & $\mathrm{D}(2,3,4,5)$ & E-ele (a.u.) & $\begin{array}{c}\text { E-ele-rel } \\
(\mathrm{kcal} / \mathrm{mol})\end{array}$ \\
\hline-180 & 50 & -272.64690 & 33.42 & -160 & 180 & -272.65020 & 31.34 & -140 & 310 & -272.64129 & 36.94 \\
\hline-180 & 60 & -272.64907 & 32.05 & -160 & 190 & -272.65051 & 31.15 & -130 & 50 & -272.64900 & 32.10 \\
\hline-180 & 70 & -272.65018 & 31.36 & -160 & 200 & -272.65080 & 30.97 & -130 & 60 & -272.64974 & 31.63 \\
\hline-180 & 80 & -272.65056 & 31.12 & -160 & 210 & -272.65102 & 30.83 & -130 & 70 & -272.65046 & 31.19 \\
\hline-180 & 90 & -272.65063 & 31.08 & -160 & 220 & -272.65119 & 30.73 & -130 & 80 & -272.65092 & 30.90 \\
\hline-180 & 100 & -272.65065 & 31.07 & -160 & 230 & -272.65129 & 30.66 & -130 & 90 & -272.65109 & 30.79 \\
\hline-180 & 110 & -272.65066 & 31.06 & -160 & 240 & -272.65137 & 30.61 & -130 & 100 & -272.65108 & 30.80 \\
\hline-180 & 120 & -272.65065 & 31.07 & -160 & 250 & -272.65146 & 30.56 & -130 & 110 & -272.65098 & 30.86 \\
\hline-180 & 130 & -272.65061 & 31.09 & -160 & 260 & -272.65150 & 30.53 & -130 & 120 & -272.65088 & 30.92 \\
\hline-180 & 140 & -272.65050 & 31.16 & -160 & 270 & -272.65139 & 30.60 & -130 & 130 & -272.65079 & 30.98 \\
\hline-180 & 150 & -272.65032 & 31.27 & -160 & 280 & -272.65082 & 30.96 & -130 & 140 & -272.65080 & 30.97 \\
\hline-180 & 160 & -272.65007 & 31.43 & -160 & 290 & -272.64935 & 31.88 & -130 & 150 & -272.65091 & 30.90 \\
\hline-180 & 170 & -272.64984 & 31.58 & -160 & 300 & -272.64671 & 33.54 & -130 & 160 & -272.65114 & 30.76 \\
\hline-180 & 180 & -272.64975 & 31.63 & -160 & 310 & -272.64129 & 36.94 & -130 & 170 & -272.65146 & 30.56 \\
\hline-180 & 190 & -272.64984 & 31.58 & -150 & 50 & -272.64900 & 32.10 & -130 & 180 & -272.65181 & 30.34 \\
\hline-180 & 200 & -272.65007 & 31.43 & -150 & 60 & -272.64957 & 31.74 & -130 & 190 & -272.65214 & 30.13 \\
\hline-180 & 210 & -272.65032 & 31.27 & -150 & 70 & -272.64997 & 31.49 & -130 & 200 & -272.65243 & 29.95 \\
\hline-180 & 220 & -272.65050 & 31.16 & -150 & 80 & -272.65027 & 31.30 & -130 & 210 & -272.65268 & 29.79 \\
\hline-180 & 230 & -272.65061 & 31.09 & -150 & 90 & -272.65049 & 31.17 & -130 & 220 & -272.65286 & 29.68 \\
\hline-180 & 240 & -272.65065 & 31.07 & -150 & 100 & -272.65059 & 31.10 & -130 & 230 & -272.65296 & 29.61 \\
\hline-180 & 250 & -272.65066 & 31.06 & -150 & 110 & -272.65059 & 31.10 & -130 & 240 & -272.65295 & 29.62 \\
\hline-180 & 260 & -272.65065 & 31.07 & -150 & 120 & -272.65049 & 31.16 & -130 & 250 & -272.65278 & 29.73 \\
\hline-180 & 270 & -272.65063 & 31.08 & -150 & 130 & -272.65033 & 31.26 & -130 & 260 & -272.65231 & 30.02 \\
\hline-180 & 280 & -272.65056 & 31.12 & -150 & 140 & -272.65016 & 31.37 & -130 & 270 & -272.65124 & 30.69 \\
\hline-180 & 290 & -272.65018 & 31.36 & -150 & 150 & -272.65005 & 31.44 & -130 & 280 & -272.64929 & 31.92 \\
\hline-180 & 300 & -272.64907 & 32.05 & -150 & 160 & -272.65010 & 31.41 & -130 & 290 & -272.64637 & 33.75 \\
\hline-180 & 310 & -272.64690 & 33.42 & -150 & 170 & -272.65033 & 31.27 & -130 & 300 & -272.64273 & 36.04 \\
\hline-170 & 50 & -272.64814 & 32.64 & -150 & 180 & -272.65067 & 31.05 & -130 & 310 & -272.64129 & 36.94 \\
\hline-170 & 60 & -272.64952 & 31.77 & -150 & 190 & -272.65098 & 30.86 & -120 & 50 & -272.64896 & 32.13 \\
\hline-170 & 70 & -272.65010 & 31.41 & -150 & 200 & -272.65126 & 30.68 & -120 & 60 & -272.64986 & 31.56 \\
\hline-170 & 80 & -272.65029 & 31.29 & -150 & 210 & -272.65149 & 30.54 & -120 & 70 & -272.65068 & 31.05 \\
\hline-170 & 90 & -272.65039 & 31.23 & -150 & 220 & -272.65166 & 30.43 & -120 & 80 & -272.65113 & 30.77 \\
\hline-170 & 100 & -272.65044 & 31.19 & -150 & 230 & -272.65181 & 30.34 & -120 & 90 & -272.65125 & 30.69 \\
\hline-170 & 110 & -272.65050 & 31.16 & -150 & 240 & -272.65193 & 30.26 & -120 & 100 & -272.65122 & 30.71 \\
\hline-170 & 120 & -272.65049 & 31.17 & -150 & 250 & -272.65201 & 30.21 & -120 & 110 & -272.65114 & 30.76 \\
\hline-170 & 130 & -272.65041 & 31.22 & -150 & 260 & -272.65197 & 30.24 & -120 & 120 & -272.65111 & 30.77 \\
\hline-170 & 140 & -272.65026 & 31.31 & -150 & 270 & -272.65161 & 30.46 & -120 & 130 & -272.65115 & 30.75 \\
\hline-170 & 150 & -272.65006 & 31.43 & -150 & 280 & -272.65053 & 31.14 & -120 & 140 & -272.65126 & 30.68 \\
\hline-170 & 160 & -272.64984 & 31.58 & -150 & 290 & -272.64840 & 32.48 & -120 & 150 & -272.65146 & 30.56 \\
\hline-170 & 170 & -272.64975 & 31.63 & -150 & 300 & -272.64514 & 34.52 & -120 & 160 & -272.65175 & 30.38 \\
\hline-170 & 180 & -272.64987 & 31.56 & -150 & 310 & -272.64129 & 36.94 & -120 & 170 & -272.65209 & 30.16 \\
\hline-170 & 190 & -272.65012 & 31.40 & -140 & 50 & -272.64902 & 32.09 & -120 & 180 & -272.65245 & 29.94 \\
\hline-170 & 200 & -272.65041 & 31.22 & -140 & 60 & -272.64961 & 31.72 & -120 & 190 & -272.65276 & 29.74 \\
\hline-170 & 210 & -272.65064 & 31.07 & -140 & 70 & -272.65015 & 31.38 & -120 & 200 & -272.65300 & 29.59 \\
\hline-170 & 220 & -272.65080 & 30.97 & -140 & 80 & -272.65055 & 31.13 & -120 & 210 & -272.65319 & 29.47 \\
\hline-170 & 230 & -272.65090 & 30.91 & -140 & 90 & -272.65079 & 30.98 & -120 & 220 & -272.65328 & 29.41 \\
\hline-170 & 240 & -272.65095 & 30.88 & -140 & 100 & -272.65084 & 30.95 & -120 & 230 & -272.65324 & 29.44 \\
\hline-170 & 250 & -272.65098 & 30.86 & -140 & 110 & -272.65078 & 30.98 & -120 & 240 & -272.65306 & 29.55 \\
\hline-170 & 260 & -272.65101 & 30.84 & -140 & 120 & -272.65066 & 31.06 & -120 & 250 & -272.65271 & 29.78 \\
\hline-170 & 270 & -272.65101 & 30.84 & -140 & 130 & -272.65051 & 31.16 & -120 & 260 & -272.65203 & 30.20 \\
\hline-170 & 280 & -272.65081 & 30.97 & -140 & 140 & -272.65040 & 31.22 & -120 & 270 & -272.65078 & 30.98 \\
\hline-170 & 290 & -272.64998 & 31.49 & -140 & 150 & -272.65042 & 31.21 & -120 & 280 & -272.64872 & 32.28 \\
\hline-170 & 300 & -272.64811 & 32.66 & -140 & 160 & -272.65057 & 31.12 & -120 & 290 & -272.64590 & 34.05 \\
\hline-170 & 310 & -272.64512 & 34.53 & -140 & 170 & -272.65086 & 30.93 & -120 & 300 & -272.64260 & 36.11 \\
\hline-160 & 50 & -272.64880 & 32.23 & -140 & 180 & -272.65121 & 30.71 & -120 & 310 & -272.64129 & 36.94 \\
\hline-160 & 60 & -272.64960 & 31.73 & -140 & 190 & -272.65153 & 30.52 & -110 & 50 & -272.64869 & 32.30 \\
\hline-160 & 70 & -272.64997 & 31.49 & -140 & 200 & -272.65182 & 30.33 & -110 & 60 & -272.64967 & 31.68 \\
\hline-160 & 80 & -272.65018 & 31.36 & -140 & 210 & -272.65206 & 30.18 & -110 & 70 & -272.65051 & 31.15 \\
\hline-160 & 90 & -272.65034 & 31.26 & -140 & 220 & -272.65226 & 30.05 & -110 & 80 & -272.65098 & 30.86 \\
\hline-160 & 100 & -272.65044 & 31.19 & -140 & 230 & -272.65242 & 29.96 & -110 & 90 & -272.65115 & 30.75 \\
\hline-160 & 110 & -272.65048 & 31.17 & -140 & 240 & -272.65251 & 29.90 & -110 & 100 & -272.65119 & 30.73 \\
\hline-160 & 120 & -272.65042 & 31.21 & -140 & 250 & -272.65250 & 29.90 & -110 & 110 & -272.65128 & 30.67 \\
\hline-160 & 130 & -272.65030 & 31.28 & -140 & 260 & -272.65228 & 30.04 & -110 & 120 & -272.65138 & 30.60 \\
\hline-160 & 140 & -272.65012 & 31.40 & -140 & 270 & -272.65155 & 30.50 & -110 & 130 & -272.65156 & 30.49 \\
\hline-160 & 150 & -272.64993 & 31.52 & -140 & 280 & -272.64997 & 31.49 & -110 & 140 & -272.65178 & 30.36 \\
\hline-160 & 160 & -272.64983 & 31.58 & -140 & 290 & -272.64731 & 33.16 & -110 & 150 & -272.65206 & 30.18 \\
\hline-160 & 170 & -272.64994 & 31.51 & -140 & 300 & -272.64370 & 35.43 & -110 & 160 & -272.65235 & 30.00 \\
\hline
\end{tabular}




\begin{tabular}{|c|c|c|c|c|c|c|c|c|c|c|c|}
\hline $\mathrm{D}(3,4,5,6)$ & $\mathrm{D}(2,3,4,5)$ & E-ele (a.u.) & $\begin{array}{c}\text { E-ele-rel } \\
(\mathrm{kcal} / \mathrm{mol}) \\
\end{array}$ & $\mathrm{D}(3,4,5,6)$ & $\mathrm{D}(2,3,4,5)$ & E-ele (a.u.) & $\begin{array}{c}\text { E-ele-rel } \\
(\mathrm{kcal} / \mathrm{mol}) \\
\end{array}$ & $\mathrm{D}(3,4,5,6)$ & $\mathrm{D}(2,3,4,5)$ & E-ele (a.u.) & $\begin{array}{c}\text { E-ele-rel } \\
(\mathrm{kcal} / \mathrm{mol}) \\
\end{array}$ \\
\hline-110 & 170 & -272.65267 & 29.80 & -80 & 70 & -272.64715 & 33.26 & -60 & 240 & -272.65111 & 30.77 \\
\hline-110 & 180 & -272.65297 & 29.61 & -80 & 80 & -272.64889 & 32.17 & -60 & 250 & -272.65114 & 30.76 \\
\hline-110 & 190 & -272.65320 & 29.46 & -80 & 90 & -272.65027 & 31.30 & -60 & 260 & -272.65122 & 30.71 \\
\hline-110 & 200 & -272.65334 & 29.38 & -80 & 100 & -272.65126 & 30.69 & -60 & 270 & -272.65125 & 30.69 \\
\hline-110 & 210 & -272.65340 & 29.34 & -80 & 110 & -272.65194 & 30.25 & -60 & 280 & -272.65113 & 30.77 \\
\hline-110 & 220 & -272.65336 & 29.37 & -80 & 120 & -272.65246 & 29.93 & -60 & 290 & -272.65068 & 31.05 \\
\hline-110 & 230 & -272.65317 & 29.49 & -80 & 130 & -272.65284 & 29.69 & -60 & 300 & -272.64986 & 31.56 \\
\hline-110 & 240 & -272.65287 & 29.68 & -80 & 140 & -272.65313 & 29.51 & -60 & 310 & -272.64896 & 32.13 \\
\hline-110 & 250 & -272.65237 & 29.99 & -80 & 150 & -272.65331 & 29.39 & -50 & 50 & -272.64129 & 36.94 \\
\hline-110 & 260 & -272.65161 & 30.46 & -80 & 160 & -272.65340 & 29.34 & -50 & 60 & -272.64273 & 36.04 \\
\hline-110 & 270 & -272.65039 & 31.23 & -80 & 170 & -272.65340 & 29.34 & -50 & 70 & -272.64637 & 33.75 \\
\hline-110 & 280 & -272.64855 & 32.38 & -80 & 180 & -272.65330 & 29.40 & -50 & 80 & -272.64929 & 31.92 \\
\hline-110 & 290 & -272.64615 & 33.89 & -80 & 190 & -272.65310 & 29.53 & -50 & 90 & -272.65124 & 30.69 \\
\hline-110 & 300 & -272.64354 & 35.53 & -80 & 200 & -272.65288 & 29.67 & -50 & 100 & -272.65231 & 30.02 \\
\hline-110 & 310 & -272.64129 & 36.94 & -80 & 210 & -272.65262 & 29.83 & -50 & 110 & -272.65278 & 29.73 \\
\hline-100 & 50 & -272.64796 & 32.75 & -80 & 220 & -272.65232 & 30.02 & -50 & 120 & -272.65295 & 29.62 \\
\hline-100 & 60 & -272.64893 & 32.14 & -80 & 230 & -272.65200 & 30.22 & -50 & 130 & -272.65296 & 29.61 \\
\hline-100 & 70 & -272.64979 & 31.61 & -80 & 240 & -272.65170 & 30.40 & -50 & 140 & -272.65286 & 29.68 \\
\hline-100 & 80 & -272.65043 & 31.20 & -80 & 250 & -272.65142 & 30.58 & -50 & 150 & -272.65268 & 29.79 \\
\hline-100 & 90 & -272.65085 & 30.94 & -80 & 260 & -272.65112 & 30.77 & -50 & 160 & -272.65243 & 29.95 \\
\hline-100 & 100 & -272.65112 & 30.77 & -80 & 270 & -272.65085 & 30.94 & -50 & 170 & -272.65214 & 30.13 \\
\hline-100 & 110 & -272.65142 & 30.58 & -80 & 280 & -272.65043 & 31.20 & -50 & 180 & -272.65181 & 30.34 \\
\hline-100 & 120 & -272.65170 & 30.40 & -80 & 290 & -272.64979 & 31.61 & -50 & 190 & -272.65146 & 30.56 \\
\hline-100 & 130 & -272.65200 & 30.22 & -80 & 300 & -272.64893 & 32.14 & -50 & 200 & -272.65114 & 30.76 \\
\hline-100 & 140 & -272.65232 & 30.02 & -80 & 310 & -272.64796 & 32.75 & -50 & 210 & -272.65091 & 30.90 \\
\hline-100 & 150 & -272.65262 & 29.83 & -70 & 50 & -272.64129 & 36.94 & -50 & 220 & -272.65080 & 30.97 \\
\hline-100 & 160 & -272.65288 & 29.67 & -70 & 60 & -272.64354 & 35.53 & -50 & 230 & -272.65079 & 30.98 \\
\hline-100 & 170 & -272.65310 & 29.53 & -70 & 70 & -272.64615 & 33.89 & -50 & 240 & -272.65088 & 30.92 \\
\hline-100 & 180 & -272.65330 & 29.40 & -70 & 80 & -272.64855 & 32.38 & -50 & 250 & -272.65098 & 30.86 \\
\hline-100 & 190 & -272.65340 & 29.34 & -70 & 90 & -272.65039 & 31.23 & -50 & 260 & -272.65108 & 30.80 \\
\hline-100 & 200 & -272.65340 & 29.34 & -70 & 100 & -272.65161 & 30.46 & -50 & 270 & -272.65109 & 30.79 \\
\hline-100 & 210 & -272.65331 & 29.39 & -70 & 110 & -272.65237 & 29.99 & -50 & 280 & -272.65092 & 30.90 \\
\hline-100 & 220 & -272.65313 & 29.51 & -70 & 120 & -272.65287 & 29.68 & -50 & 290 & -272.65046 & 31.19 \\
\hline-100 & 230 & -272.65284 & 29.69 & -70 & 130 & -272.65317 & 29.49 & -50 & 300 & -272.64974 & 31.63 \\
\hline-100 & 240 & -272.65246 & 29.93 & -70 & 140 & -272.65336 & 29.37 & -50 & 310 & -272.64900 & 32.10 \\
\hline-100 & 250 & -272.65194 & 30.25 & -70 & 150 & -272.65340 & 29.34 & -40 & 50 & -272.64129 & 36.94 \\
\hline-100 & 260 & -272.65126 & 30.69 & -70 & 160 & -272.65334 & 29.38 & -40 & 60 & -272.64370 & 35.43 \\
\hline-100 & 270 & -272.65027 & 31.30 & -70 & 170 & -272.65320 & 29.46 & -40 & 70 & -272.64731 & 33.16 \\
\hline-100 & 280 & -272.64889 & 32.17 & -70 & 180 & -272.65297 & 29.61 & -40 & 80 & -272.64997 & 31.49 \\
\hline-100 & 290 & -272.64715 & 33.26 & -70 & 190 & -272.65267 & 29.80 & -40 & 90 & -272.65155 & 30.50 \\
\hline-100 & 300 & -272.64539 & 34.37 & -70 & 200 & -272.65235 & 30.00 & -40 & 100 & -272.65228 & 30.04 \\
\hline-100 & 310 & -272.64129 & 36.94 & -70 & 210 & -272.65206 & 30.18 & -40 & 110 & -272.65250 & 29.90 \\
\hline-90 & 50 & -272.64661 & 33.60 & -70 & 220 & -272.65178 & 30.36 & -40 & 120 & -272.65251 & 29.90 \\
\hline-90 & 60 & -272.64755 & 33.01 & -70 & 230 & -272.65156 & 30.49 & -40 & 130 & -272.65242 & 29.96 \\
\hline-90 & 70 & -272.64861 & 32.34 & -70 & 240 & -272.65138 & 30.60 & -40 & 140 & -272.65226 & 30.05 \\
\hline-90 & 80 & -272.64966 & 31.69 & -70 & 250 & -272.65128 & 30.67 & -40 & 150 & -272.65206 & 30.18 \\
\hline-90 & 90 & -272.65049 & 31.16 & -70 & 260 & -272.65119 & 30.73 & -40 & 160 & -272.65182 & 30.33 \\
\hline-90 & 100 & -272.65112 & 30.77 & -70 & 270 & -272.65115 & 30.75 & -40 & 170 & -272.65153 & 30.52 \\
\hline-90 & 110 & -272.65164 & 30.45 & -70 & 280 & -272.65098 & 30.86 & -40 & 180 & -272.65121 & 30.71 \\
\hline-90 & 120 & -272.65208 & 30.17 & -70 & 290 & -272.65051 & 31.15 & -40 & 190 & -272.65086 & 30.93 \\
\hline-90 & 130 & -272.65247 & 29.93 & -70 & 300 & -272.64967 & 31.68 & -40 & 200 & -272.65057 & 31.12 \\
\hline-90 & 140 & -272.65281 & 29.71 & -70 & 310 & -272.64869 & 32.30 & -40 & 210 & -272.65042 & 31.21 \\
\hline-90 & 150 & -272.65307 & 29.55 & -60 & 50 & -272.64129 & 36.94 & -40 & 220 & -272.65040 & 31.22 \\
\hline-90 & 160 & -272.65324 & 29.44 & -60 & 60 & -272.64260 & 36.11 & -40 & 230 & -272.65051 & 31.16 \\
\hline-90 & 170 & -272.65335 & 29.37 & -60 & 70 & -272.64590 & 34.05 & -40 & 240 & -272.65066 & 31.06 \\
\hline-90 & 180 & -272.65340 & 29.34 & -60 & 80 & -272.64872 & 32.28 & -40 & 250 & -272.65078 & 30.98 \\
\hline-90 & 190 & -272.65335 & 29.37 & -60 & 90 & -272.65078 & 30.98 & -40 & 260 & -272.65084 & 30.95 \\
\hline-90 & 200 & -272.65324 & 29.44 & -60 & 100 & -272.65203 & 30.20 & -40 & 270 & -272.65079 & 30.98 \\
\hline-90 & 210 & -272.65307 & 29.55 & -60 & 110 & -272.65271 & 29.78 & -40 & 280 & -272.65055 & 31.13 \\
\hline-90 & 220 & -272.65281 & 29.71 & -60 & 120 & -272.65306 & 29.55 & -40 & 290 & -272.65015 & 31.38 \\
\hline-90 & 230 & -272.65247 & 29.93 & -60 & 130 & -272.65324 & 29.44 & -40 & 300 & -272.64961 & 31.72 \\
\hline-90 & 240 & -272.65208 & 30.17 & -60 & 140 & -272.65328 & 29.41 & -40 & 310 & -272.64902 & 32.09 \\
\hline-90 & 250 & -272.65164 & 30.45 & -60 & 150 & -272.65319 & 29.47 & -30 & 50 & -272.64129 & 36.94 \\
\hline-90 & 260 & -272.65112 & 30.77 & -60 & 160 & -272.65300 & 29.59 & -30 & 60 & -272.64514 & 34.52 \\
\hline-90 & 270 & -272.65049 & 31.16 & -60 & 170 & -272.65276 & 29.74 & -30 & 70 & -272.64840 & 32.48 \\
\hline-90 & 280 & -272.64966 & 31.69 & -60 & 180 & -272.65245 & 29.94 & -30 & 80 & -272.65053 & 31.14 \\
\hline-90 & 290 & -272.64861 & 32.34 & -60 & 190 & -272.65209 & 30.16 & -30 & 90 & -272.65161 & 30.46 \\
\hline-90 & 300 & -272.64755 & 33.01 & -60 & 200 & -272.65175 & 30.38 & -30 & 100 & -272.65197 & 30.24 \\
\hline-90 & 310 & -272.64661 & 33.60 & -60 & 210 & -272.65146 & 30.56 & -30 & 110 & -272.65201 & 30.21 \\
\hline-80 & 50 & -272.64129 & 36.94 & -60 & 220 & -272.65126 & 30.68 & -30 & 120 & -272.65193 & 30.26 \\
\hline-80 & 60 & -272.64539 & 34.37 & -60 & 230 & -272.65115 & 30.75 & -30 & 130 & -272.65181 & 30.34 \\
\hline
\end{tabular}




\begin{tabular}{|c|c|c|c|c|c|c|c|c|c|c|c|}
\hline $\mathrm{D}(3,4,5,6)$ & $\mathrm{D}(2,3,4,5)$ & E-ele (a.u.) & $\begin{array}{c}\text { E-ele-rel } \\
(\mathrm{kcal} / \mathrm{mol}) \\
\end{array}$ & $\mathrm{D}(3,4,5,6)$ & $\mathrm{D}(2,3,4,5)$ & E-ele (a.u.) & $\begin{array}{c}\text { E-ele-rel } \\
(\mathrm{kcal} / \mathrm{mol}) \\
\end{array}$ & $\mathrm{D}(3,4,5,6)$ & $\mathrm{D}(2,3,4,5)$ & E-ele (a.u.) & $\begin{array}{c}\text { E-ele-rel } \\
(\mathrm{kcal} / \mathrm{mol}) \\
\end{array}$ \\
\hline-30 & 140 & -272.65166 & 30.43 & -10 & 310 & -272.64814 & 32.64 & 20 & 210 & -272.65102 & 30.83 \\
\hline-30 & 150 & -272.65149 & 30.54 & 0 & 50 & -272.64690 & 33.42 & 20 & 220 & -272.65119 & 30.73 \\
\hline-30 & 160 & -272.65126 & 30.68 & 0 & 60 & -272.64907 & 32.05 & 20 & 230 & -272.65129 & 30.66 \\
\hline-30 & 170 & -272.65098 & 30.86 & 0 & 70 & -272.65018 & 31.36 & 20 & 240 & -272.65137 & 30.61 \\
\hline-30 & 180 & -272.65067 & 31.05 & 0 & 80 & -272.65056 & 31.12 & 20 & 250 & -272.65146 & 30.56 \\
\hline-30 & 190 & -272.65033 & 31.27 & 0 & 90 & -272.65063 & 31.08 & 20 & 260 & -272.65150 & 30.53 \\
\hline-30 & 200 & -272.65010 & 31.41 & 0 & 100 & -272.65065 & 31.07 & 20 & 270 & -272.65139 & 30.60 \\
\hline-30 & 210 & -272.65005 & 31.44 & 0 & 110 & -272.65066 & 31.06 & 20 & 280 & -272.65082 & 30.96 \\
\hline-30 & 220 & -272.65016 & 31.37 & 0 & 120 & -272.65065 & 31.07 & 20 & 290 & -272.64935 & 31.88 \\
\hline-30 & 230 & -272.65033 & 31.26 & 0 & 130 & -272.65061 & 31.09 & 20 & 300 & -272.64671 & 33.54 \\
\hline-30 & 240 & -272.65049 & 31.16 & 0 & 140 & -272.65050 & 31.16 & 20 & 310 & -272.64129 & 36.94 \\
\hline-30 & 250 & -272.65059 & 31.10 & 0 & 150 & -272.65032 & 31.27 & 30 & 50 & -272.64900 & 32.10 \\
\hline-30 & 260 & -272.65059 & 31.10 & 0 & 160 & -272.65007 & 31.43 & 30 & 60 & -272.64957 & 31.74 \\
\hline-30 & 270 & -272.65049 & 31.17 & 0 & 170 & -272.64984 & 31.58 & 30 & 70 & -272.64997 & 31.49 \\
\hline-30 & 280 & -272.65027 & 31.30 & 0 & 180 & -272.64975 & 31.63 & 30 & 80 & -272.65027 & 31.30 \\
\hline-30 & 290 & -272.64997 & 31.49 & 0 & 190 & -272.64984 & 31.58 & 30 & 90 & -272.65049 & 31.17 \\
\hline-30 & 300 & -272.64957 & 31.74 & 0 & 200 & -272.65007 & 31.43 & 30 & 100 & -272.65059 & 31.10 \\
\hline-30 & 310 & -272.64900 & 32.10 & 0 & 210 & -272.65032 & 31.27 & 30 & 110 & -272.65059 & 31.10 \\
\hline-20 & 50 & -272.64129 & 36.94 & 0 & 220 & -272.65050 & 31.16 & 30 & 120 & -272.65049 & 31.16 \\
\hline-20 & 60 & -272.64671 & 33.54 & 0 & 230 & -272.65061 & 31.09 & 30 & 130 & -272.65033 & 31.26 \\
\hline-20 & 70 & -272.64935 & 31.88 & 0 & 240 & -272.65065 & 31.07 & 30 & 140 & -272.65016 & 31.37 \\
\hline-20 & 80 & -272.65082 & 30.96 & 0 & 250 & -272.65066 & 31.06 & 30 & 150 & -272.65005 & 31.44 \\
\hline-20 & 90 & -272.65139 & 30.60 & 0 & 260 & -272.65065 & 31.07 & 30 & 160 & -272.65010 & 31.41 \\
\hline-20 & 100 & -272.65150 & 30.53 & 0 & 270 & -272.65063 & 31.08 & 30 & 170 & -272.65033 & 31.27 \\
\hline-20 & 110 & -272.65146 & 30.56 & 0 & 280 & -272.65056 & 31.12 & 30 & 180 & -272.65067 & 31.05 \\
\hline-20 & 120 & -272.65137 & 30.61 & 0 & 290 & -272.65018 & 31.36 & 30 & 190 & -272.65098 & 30.86 \\
\hline-20 & 130 & -272.65129 & 30.66 & 0 & 300 & -272.64907 & 32.05 & 30 & 200 & -272.65126 & 30.68 \\
\hline-20 & 140 & -272.65119 & 30.73 & 0 & 310 & -272.64690 & 33.42 & 30 & 210 & -272.65149 & 30.54 \\
\hline-20 & 150 & -272.65102 & 30.83 & 10 & 50 & -272.64814 & 32.64 & 30 & 220 & -272.65166 & 30.43 \\
\hline-20 & 160 & -272.65080 & 30.97 & 10 & 60 & -272.64952 & 31.77 & 30 & 230 & -272.65181 & 30.34 \\
\hline-20 & 170 & -272.65051 & 31.15 & 10 & 70 & -272.65010 & 31.41 & 30 & 240 & -272.65193 & 30.26 \\
\hline-20 & 180 & -272.65020 & 31.34 & 10 & 80 & -272.65029 & 31.29 & 30 & 250 & -272.65201 & 30.21 \\
\hline-20 & 190 & -272.64994 & 31.51 & 10 & 90 & -272.65039 & 31.23 & 30 & 260 & -272.65197 & 30.24 \\
\hline-20 & 200 & -272.64983 & 31.58 & 10 & 100 & -272.65044 & 31.19 & 30 & 270 & -272.65161 & 30.46 \\
\hline-20 & 210 & -272.64993 & 31.52 & 10 & 110 & -272.65050 & 31.16 & 30 & 280 & -272.65053 & 31.14 \\
\hline-20 & 220 & -272.65012 & 31.40 & 10 & 120 & -272.65049 & 31.17 & 30 & 290 & -272.64840 & 32.48 \\
\hline-20 & 230 & -272.65030 & 31.28 & 10 & 130 & -272.65041 & 31.22 & 30 & 300 & -272.64514 & 34.52 \\
\hline-20 & 240 & -272.65042 & 31.21 & 10 & 140 & -272.65026 & 31.31 & 30 & 310 & -272.64129 & 36.94 \\
\hline-20 & 250 & -272.65048 & 31.17 & 10 & 150 & -272.65006 & 31.43 & 40 & 50 & -272.64902 & 32.09 \\
\hline-20 & 260 & -272.65044 & 31.19 & 10 & 160 & -272.64984 & 31.58 & 40 & 60 & -272.64961 & 31.72 \\
\hline-20 & 270 & -272.65034 & 31.26 & 10 & 170 & -272.64975 & 31.63 & 40 & 70 & -272.65015 & 31.38 \\
\hline-20 & 280 & -272.65018 & 31.36 & 10 & 180 & -272.64987 & 31.56 & 40 & 80 & -272.65055 & 31.13 \\
\hline-20 & 290 & -272.64997 & 31.49 & 10 & 190 & -272.65012 & 31.40 & 40 & 90 & -272.65079 & 30.98 \\
\hline-20 & 300 & -272.64960 & 31.73 & 10 & 200 & -272.65041 & 31.22 & 40 & 100 & -272.65084 & 30.95 \\
\hline-20 & 310 & -272.64880 & 32.23 & 10 & 210 & -272.65064 & 31.07 & 40 & 110 & -272.65078 & 30.98 \\
\hline-10 & 50 & -272.64512 & 34.53 & 10 & 220 & -272.65080 & 30.97 & 40 & 120 & -272.65066 & 31.06 \\
\hline-10 & 60 & -272.64811 & 32.66 & 10 & 230 & -272.65090 & 30.91 & 40 & 130 & -272.65051 & 31.16 \\
\hline-10 & 70 & -272.64998 & 31.49 & 10 & 240 & -272.65095 & 30.88 & 40 & 140 & -272.65040 & 31.22 \\
\hline-10 & 80 & -272.65081 & 30.97 & 10 & 250 & -272.65098 & 30.86 & 40 & 150 & -272.65042 & 31.21 \\
\hline-10 & 90 & -272.65101 & 30.84 & 10 & 260 & -272.65101 & 30.84 & 40 & 160 & -272.65057 & 31.12 \\
\hline-10 & 100 & -272.65101 & 30.84 & 10 & 270 & -272.65101 & 30.84 & 40 & 170 & -272.65086 & 30.93 \\
\hline-10 & 110 & -272.65098 & 30.86 & 10 & 280 & -272.65081 & 30.97 & 40 & 180 & -272.65121 & 30.71 \\
\hline-10 & 120 & -272.65095 & 30.88 & 10 & 290 & -272.64998 & 31.49 & 40 & 190 & -272.65153 & 30.52 \\
\hline-10 & 130 & -272.65090 & 30.91 & 10 & 300 & -272.64811 & 32.66 & 40 & 200 & -272.65182 & 30.33 \\
\hline-10 & 140 & -272.65080 & 30.97 & 10 & 310 & -272.64512 & 34.53 & 40 & 210 & -272.65206 & 30.18 \\
\hline-10 & 150 & -272.65064 & 31.07 & 20 & 50 & -272.64880 & 32.23 & 40 & 220 & -272.65226 & 30.05 \\
\hline-10 & 160 & -272.65041 & 31.22 & 20 & 60 & -272.64960 & 31.73 & 40 & 230 & -272.65242 & 29.96 \\
\hline-10 & 170 & -272.65012 & 31.40 & 20 & 70 & -272.64997 & 31.49 & 40 & 240 & -272.65251 & 29.90 \\
\hline-10 & 180 & -272.64987 & 31.56 & 20 & 80 & -272.65018 & 31.36 & 40 & 250 & -272.65250 & 29.90 \\
\hline-10 & 190 & -272.64975 & 31.63 & 20 & 90 & -272.65034 & 31.26 & 40 & 260 & -272.65228 & 30.04 \\
\hline-10 & 200 & -272.64984 & 31.58 & 20 & 100 & -272.65044 & 31.19 & 40 & 270 & -272.65155 & 30.50 \\
\hline-10 & 210 & -272.65006 & 31.43 & 20 & 110 & -272.65048 & 31.17 & 40 & 280 & -272.64997 & 31.49 \\
\hline-10 & 220 & -272.65026 & 31.31 & 20 & 120 & -272.65042 & 31.21 & 40 & 290 & -272.64731 & 33.16 \\
\hline-10 & 230 & -272.65041 & 31.22 & 20 & 130 & -272.65030 & 31.28 & 40 & 300 & -272.64370 & 35.43 \\
\hline-10 & 240 & -272.65049 & 31.17 & 20 & 140 & -272.65012 & 31.40 & 40 & 310 & -272.64129 & 36.94 \\
\hline-10 & 250 & -272.65050 & 31.16 & 20 & 150 & -272.64993 & 31.52 & 50 & 50 & -272.64900 & 32.10 \\
\hline-10 & 260 & -272.65044 & 31.19 & 20 & 160 & -272.64983 & 31.58 & 50 & 60 & -272.64974 & 31.63 \\
\hline-10 & 270 & -272.65039 & 31.23 & 20 & 170 & -272.64994 & 31.51 & 50 & 70 & -272.65046 & 31.19 \\
\hline-10 & 280 & -272.65029 & 31.29 & 20 & 180 & -272.65020 & 31.34 & 50 & 80 & -272.65092 & 30.90 \\
\hline-10 & 290 & -272.65010 & 31.41 & 20 & 190 & -272.65051 & 31.15 & 50 & 90 & -272.65109 & 30.79 \\
\hline-10 & 300 & -272.64952 & 31.77 & 20 & 200 & -272.65080 & 30.97 & 50 & 100 & -272.65108 & 30.80 \\
\hline
\end{tabular}




\begin{tabular}{|c|c|c|c|c|c|c|c|c|c|c|c|}
\hline $\mathrm{D}(3,4,5,6)$ & $\mathrm{D}(2,3,4,5)$ & E-ele (a.u.) & $\begin{array}{c}\text { E-ele-rel } \\
(\mathrm{kcal} / \mathrm{mol}) \\
\end{array}$ & $\mathrm{D}(3,4,5,6)$ & $\mathrm{D}(2,3,4,5)$ & E-ele (a.u.) & $\begin{array}{c}\text { E-ele-rel } \\
(\mathrm{kcal} / \mathrm{mol}) \\
\end{array}$ & $\mathrm{D}(3,4,5,6)$ & $\mathrm{D}(2,3,4,5)$ & E-ele (a.u.) & $\begin{array}{c}\text { E-ele-rel } \\
(\mathrm{kcal} / \mathrm{mol})\end{array}$ \\
\hline 50 & 110 & -272.65098 & 30.86 & 70 & 280 & -272.64855 & 32.38 & 100 & 180 & -272.65330 & 29.40 \\
\hline 50 & 120 & -272.65088 & 30.92 & 70 & 290 & -272.64615 & 33.89 & 100 & 190 & -272.65310 & 29.53 \\
\hline 50 & 130 & -272.65079 & 30.98 & 70 & 300 & -272.64354 & 35.53 & 100 & 200 & -272.65288 & 29.67 \\
\hline 50 & 140 & -272.65080 & 30.97 & 70 & 310 & -272.64129 & 36.94 & 100 & 210 & -272.65262 & 29.83 \\
\hline 50 & 150 & -272.65091 & 30.90 & 80 & 50 & -272.64796 & 32.75 & 100 & 220 & -272.65232 & 30.02 \\
\hline 50 & 160 & -272.65114 & 30.76 & 80 & 60 & -272.64893 & 32.14 & 100 & 230 & -272.65200 & 30.22 \\
\hline 50 & 170 & -272.65146 & 30.56 & 80 & 70 & -272.64979 & 31.61 & 100 & 240 & -272.65170 & 30.40 \\
\hline 50 & 180 & -272.65181 & 30.34 & 80 & 80 & -272.65043 & 31.20 & 100 & 250 & -272.65142 & 30.58 \\
\hline 50 & 190 & -272.65214 & 30.13 & 80 & 90 & -272.65085 & 30.94 & 100 & 260 & -272.65112 & 30.77 \\
\hline 50 & 200 & -272.65243 & 29.95 & 80 & 100 & -272.65112 & 30.77 & 100 & 270 & -272.65085 & 30.94 \\
\hline 50 & 210 & -272.65268 & 29.79 & 80 & 110 & -272.65142 & 30.58 & 100 & 280 & -272.65043 & 31.20 \\
\hline 50 & 220 & -272.65286 & 29.68 & 80 & 120 & -272.65170 & 30.40 & 100 & 290 & -272.64979 & 31.61 \\
\hline 50 & 230 & -272.65296 & 29.61 & 80 & 130 & -272.65200 & 30.22 & 100 & 300 & -272.64893 & 32.14 \\
\hline 50 & 240 & -272.65295 & 29.62 & 80 & 140 & -272.65232 & 30.02 & 100 & 310 & -272.64796 & 32.75 \\
\hline 50 & 250 & -272.65278 & 29.73 & 80 & 150 & -272.65262 & 29.83 & 110 & 50 & -272.64129 & 36.94 \\
\hline 50 & 260 & -272.65231 & 30.02 & 80 & 160 & -272.65288 & 29.67 & 110 & 60 & -272.64354 & 35.53 \\
\hline 50 & 270 & -272.65124 & 30.69 & 80 & 170 & -272.65310 & 29.53 & 110 & 70 & -272.64615 & 33.89 \\
\hline 50 & 280 & -272.64929 & 31.92 & 80 & 180 & -272.65330 & 29.40 & 110 & 80 & -272.64855 & 32.38 \\
\hline 50 & 290 & -272.64637 & 33.75 & 80 & 190 & -272.65340 & 29.34 & 110 & 90 & -272.65039 & 31.23 \\
\hline 50 & 300 & -272.64273 & 36.04 & 80 & 200 & -272.65340 & 29.34 & 110 & 100 & -272.65161 & 30.46 \\
\hline 50 & 310 & -272.64129 & 36.94 & 80 & 210 & -272.65331 & 29.39 & 110 & 110 & -272.65237 & 29.99 \\
\hline 60 & 50 & -272.64896 & 32.13 & 80 & 220 & -272.65313 & 29.51 & 110 & 120 & -272.65287 & 29.68 \\
\hline 60 & 60 & -272.64986 & 31.56 & 80 & 230 & -272.65284 & 29.69 & 110 & 130 & -272.65317 & 29.49 \\
\hline 60 & 70 & -272.65068 & 31.05 & 80 & 240 & -272.65246 & 29.93 & 110 & 140 & -272.65336 & 29.37 \\
\hline 60 & 80 & -272.65113 & 30.77 & 80 & 250 & -272.65194 & 30.25 & 110 & 150 & -272.65340 & 29.34 \\
\hline 60 & 90 & -272.65125 & 30.69 & 80 & 260 & -272.65126 & 30.69 & 110 & 160 & -272.65334 & 29.38 \\
\hline 60 & 100 & -272.65122 & 30.71 & 80 & 270 & -272.65027 & 31.30 & 110 & 170 & -272.65320 & 29.46 \\
\hline 60 & 110 & -272.65114 & 30.76 & 80 & 280 & -272.64889 & 32.17 & 110 & 180 & -272.65297 & 29.61 \\
\hline 60 & 120 & -272.65111 & 30.77 & 80 & 290 & -272.64715 & 33.26 & 110 & 190 & -272.65267 & 29.80 \\
\hline 60 & 130 & -272.65115 & 30.75 & 80 & 300 & -272.64539 & 34.37 & 110 & 200 & -272.65235 & 30.00 \\
\hline 60 & 140 & -272.65126 & 30.68 & 80 & 310 & -272.64129 & 36.94 & 110 & 210 & -272.65206 & 30.18 \\
\hline 60 & 150 & -272.65146 & 30.56 & 90 & 50 & -272.64661 & 33.60 & 110 & 220 & -272.65178 & 30.36 \\
\hline 60 & 160 & -272.65175 & 30.38 & 90 & 60 & -272.64755 & 33.01 & 110 & 230 & -272.65156 & 30.49 \\
\hline 60 & 170 & -272.65209 & 30.16 & 90 & 70 & -272.64861 & 32.34 & 110 & 240 & -272.65138 & 30.60 \\
\hline 60 & 180 & -272.65245 & 29.94 & 90 & 80 & -272.64966 & 31.69 & 110 & 250 & -272.65128 & 30.67 \\
\hline 60 & 190 & -272.65276 & 29.74 & 90 & 90 & -272.65049 & 31.16 & 110 & 260 & -272.65119 & 30.73 \\
\hline 60 & 200 & -272.65300 & 29.59 & 90 & 100 & -272.65112 & 30.77 & 110 & 270 & -272.65115 & 30.75 \\
\hline 60 & 210 & -272.65319 & 29.47 & 90 & 110 & -272.65164 & 30.45 & 110 & 280 & -272.65098 & 30.86 \\
\hline 60 & 220 & -272.65328 & 29.41 & 90 & 120 & -272.65208 & 30.17 & 110 & 290 & -272.65051 & 31.15 \\
\hline 60 & 230 & -272.65324 & 29.44 & 90 & 130 & -272.65247 & 29.93 & 110 & 300 & -272.64967 & 31.68 \\
\hline 60 & 240 & -272.65306 & 29.55 & 90 & 140 & -272.65281 & 29.71 & 110 & 310 & -272.64869 & 32.30 \\
\hline 60 & 250 & -272.65271 & 29.78 & 90 & 150 & -272.65307 & 29.55 & 120 & 50 & -272.64129 & 36.94 \\
\hline 60 & 260 & -272.65203 & 30.20 & 90 & 160 & -272.65324 & 29.44 & 120 & 60 & -272.64260 & 36.11 \\
\hline 60 & 270 & -272.65078 & 30.98 & 90 & 170 & -272.65335 & 29.37 & 120 & 70 & -272.64590 & 34.05 \\
\hline 60 & 280 & -272.64872 & 32.28 & 90 & 180 & -272.65340 & 29.34 & 120 & 80 & -272.64872 & 32.28 \\
\hline 60 & 290 & -272.64590 & 34.05 & 90 & 190 & -272.65335 & 29.37 & 120 & 90 & -272.65078 & 30.98 \\
\hline 60 & 300 & -272.64260 & 36.11 & 90 & 200 & -272.65324 & 29.44 & 120 & 100 & -272.65203 & 30.20 \\
\hline 60 & 310 & -272.64129 & 36.94 & 90 & 210 & -272.65307 & 29.55 & 120 & 110 & -272.65271 & 29.78 \\
\hline 70 & 50 & -272.64869 & 32.30 & 90 & 220 & -272.65281 & 29.71 & 120 & 120 & -272.65306 & 29.55 \\
\hline 70 & 60 & -272.64967 & 31.68 & 90 & 230 & -272.65247 & 29.93 & 120 & 130 & -272.65324 & 29.44 \\
\hline 70 & 70 & -272.65051 & 31.15 & 90 & 240 & -272.65208 & 30.17 & 120 & 140 & -272.65328 & 29.41 \\
\hline 70 & 80 & -272.65098 & 30.86 & 90 & 250 & -272.65164 & 30.45 & 120 & 150 & -272.65319 & 29.47 \\
\hline 70 & 90 & -272.65115 & 30.75 & 90 & 260 & -272.65112 & 30.77 & 120 & 160 & -272.65300 & 29.59 \\
\hline 70 & 100 & -272.65119 & 30.73 & 90 & 270 & -272.65049 & 31.16 & 120 & 170 & -272.65276 & 29.74 \\
\hline 70 & 110 & -272.65128 & 30.67 & 90 & 280 & -272.64966 & 31.69 & 120 & 180 & -272.65245 & 29.94 \\
\hline 70 & 120 & -272.65138 & 30.60 & 90 & 290 & -272.64861 & 32.34 & 120 & 190 & -272.65209 & 30.16 \\
\hline 70 & 130 & -272.65156 & 30.49 & 90 & 300 & -272.64755 & 33.01 & 120 & 200 & -272.65175 & 30.38 \\
\hline 70 & 140 & -272.65178 & 30.36 & 90 & 310 & -272.64661 & 33.60 & 120 & 210 & -272.65146 & 30.56 \\
\hline 70 & 150 & -272.65206 & 30.18 & 100 & 50 & -272.64129 & 36.94 & 120 & 220 & -272.65126 & 30.68 \\
\hline 70 & 160 & -272.65235 & 30.00 & 100 & 60 & -272.64539 & 34.37 & 120 & 230 & -272.65115 & 30.75 \\
\hline 70 & 170 & -272.65267 & 29.80 & 100 & 70 & -272.64715 & 33.26 & 120 & 240 & -272.65111 & 30.77 \\
\hline 70 & 180 & -272.65297 & 29.61 & 100 & 80 & -272.64889 & 32.17 & 120 & 250 & -272.65114 & 30.76 \\
\hline 70 & 190 & -272.65320 & 29.46 & 100 & 90 & -272.65027 & 31.30 & 120 & 260 & -272.65122 & 30.71 \\
\hline 70 & 200 & -272.65334 & 29.38 & 100 & 100 & -272.65126 & 30.69 & 120 & 270 & -272.65125 & 30.69 \\
\hline 70 & 210 & -272.65340 & 29.34 & 100 & 110 & -272.65194 & 30.25 & 120 & 280 & -272.65113 & 30.77 \\
\hline 70 & 220 & -272.65336 & 29.37 & 100 & 120 & -272.65246 & 29.93 & 120 & 290 & -272.65068 & 31.05 \\
\hline 70 & 230 & -272.65317 & 29.49 & 100 & 130 & -272.65284 & 29.69 & 120 & 300 & -272.64986 & 31.56 \\
\hline 70 & 240 & -272.65287 & 29.68 & 100 & 140 & -272.65313 & 29.51 & 120 & 310 & -272.64896 & 32.13 \\
\hline 70 & 250 & -272.65237 & 29.99 & 100 & 150 & -272.65331 & 29.39 & 130 & 50 & -272.64129 & 36.94 \\
\hline 70 & 260 & -272.65161 & 30.46 & 100 & 160 & -272.65340 & 29.34 & 130 & 60 & -272.64273 & 36.04 \\
\hline 70 & 270 & -272.65039 & 31.23 & 100 & 170 & -272.65340 & 29.34 & 130 & 70 & -272.64637 & 33.75 \\
\hline
\end{tabular}




\begin{tabular}{|c|c|c|c|c|c|c|c|c|c|c|c|}
\hline $\mathrm{D}(3,4,5,6)$ & $\mathrm{D}(2,3,4,5)$ & E-ele (a.u.) & $\begin{array}{c}\text { E-ele-rel } \\
(\mathrm{kcal} / \mathrm{mol})\end{array}$ & $\mathrm{D}(3,4,5,6)$ & $\mathrm{D}(2,3,4,5)$ & E-ele (a.u.) & $\begin{array}{c}\text { E-ele-rel } \\
(\mathrm{kcal} / \mathrm{mol})\end{array}$ & $\mathrm{D}(3,4,5,6)$ & $\mathrm{D}(2,3,4,5)$ & E-ele (a.u.) & $\begin{array}{c}\text { E-ele-rel } \\
(\mathrm{kcal} / \mathrm{mol})\end{array}$ \\
\hline 130 & 80 & -272.64929 & 31.92 & 150 & 250 & -272.65059 & 31.10 & 180 & 150 & -272.65032 & 31.27 \\
\hline 130 & 90 & -272.65124 & 30.69 & 150 & 260 & -272.65059 & 31.10 & 180 & 160 & -272.65007 & 31.43 \\
\hline 130 & 100 & -272.65231 & 30.02 & 150 & 270 & -272.65049 & 31.17 & 180 & 170 & -272.64984 & 31.58 \\
\hline 130 & 110 & -272.65278 & 29.73 & 150 & 280 & -272.65027 & 31.30 & 180 & 180 & -272.64975 & 31.63 \\
\hline 130 & 120 & -272.65295 & 29.62 & 150 & 290 & -272.64997 & 31.49 & 180 & 190 & -272.64984 & 31.58 \\
\hline 130 & 130 & -272.65296 & 29.61 & 150 & 300 & -272.64957 & 31.74 & 180 & 200 & -272.65007 & 31.43 \\
\hline 130 & 140 & -272.65286 & 29.68 & 150 & 310 & -272.64900 & 32.10 & 180 & 210 & -272.65032 & 31.27 \\
\hline 130 & 150 & -272.65268 & 29.79 & 160 & 50 & -272.64129 & 36.94 & 180 & 220 & -272.65050 & 31.16 \\
\hline 130 & 160 & -272.65243 & 29.95 & 160 & 60 & -272.64671 & 33.54 & 180 & 230 & -272.65061 & 31.09 \\
\hline 130 & 170 & -272.65214 & 30.13 & 160 & 70 & -272.64935 & 31.88 & 180 & 240 & -272.65065 & 31.07 \\
\hline 130 & 180 & -272.65181 & 30.34 & 160 & 80 & -272.65082 & 30.96 & 180 & 250 & -272.65066 & 31.06 \\
\hline 130 & 190 & -272.65146 & 30.56 & 160 & 90 & -272.65139 & 30.60 & 180 & 260 & -272.65065 & 31.07 \\
\hline 130 & 200 & -272.65114 & 30.76 & 160 & 100 & -272.65150 & 30.53 & 180 & 270 & -272.65063 & 31.08 \\
\hline 130 & 210 & -272.65091 & 30.90 & 160 & 110 & -272.65146 & 30.56 & 180 & 280 & -272.65056 & 31.12 \\
\hline 130 & 220 & -272.65080 & 30.97 & 160 & 120 & -272.65137 & 30.61 & 180 & 290 & -272.65018 & 31.36 \\
\hline 130 & 230 & -272.65079 & 30.98 & 160 & 130 & -272.65129 & 30.66 & 180 & 300 & -272.64907 & 32.05 \\
\hline 130 & 240 & -272.65088 & 30.92 & 160 & 140 & -272.65119 & 30.73 & 180 & 310 & -272.64690 & 33.42 \\
\hline
\end{tabular}

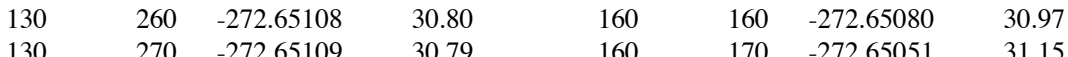

$\begin{array}{llllllll}130 & 280 & -272.65092 & 30.90 & 160 & 180 & -272.65020 & 31.34\end{array}$

$\begin{array}{llllllll}130 & 290 & -272.65046 & 31.19 & 160 & 190 & -272.64994 & 31.51\end{array}$

$\begin{array}{llllllll}130 & 300 & -272.64974 & 31.63 & 160 & 200 & -272.64983 & 31.58\end{array}$

$\begin{array}{llllllll}130 & 310 & -272.64900 & 32.10 & 160 & 210 & -272.64993 & 31.52\end{array}$

$\begin{array}{llllllll}140 & 50 & -272.64129 & 36.94 & 160 & 220 & -272.65012 & 31.40\end{array}$

$\begin{array}{llllllll}140 & 60 & -272.64370 & 35.43 & 160 & 230 & -272.65030 & 31.28\end{array}$

$\begin{array}{llllllll}140 & 70 & -272.64731 & 33.16 & 160 & 240 & -272.65042 & 31.21\end{array}$

$\begin{array}{llllllll}140 & 80 & -272.64997 & 31.49 & 160 & 250 & -272.65048 & 31.17\end{array}$

$\begin{array}{lrrrrrrr}140 & 90 & -272.65155 & 30.50 & 160 & 260 & -272.65044 & 31.19\end{array}$

$\begin{array}{llllllll}140 & 100 & -272.65228 & 30.04 & 160 & 270 & -272.65034 & 31.26\end{array}$

$\begin{array}{llllllll}140 & 110 & -272.65250 & 29.90 & 160 & 280 & -272.65018 & 31.36\end{array}$

$\begin{array}{llllllll}140 & 120 & -272.65251 & 29.90 & 160 & 290 & -272.64997 & 31.49\end{array}$

$\begin{array}{llllllll}140 & 130 & -272.65242 & 29.96 & 160 & 300 & -272.64960 & 31.73\end{array}$

$\begin{array}{llllllll}140 & 140 & -272.65226 & 30.05 & 160 & 310 & -272.64880 & 32.23\end{array}$

$\begin{array}{llllllll}140 & 150 & -272.65206 & 30.18 & 170 & 50 & -272.64512 & 34.53\end{array}$

$\begin{array}{llllllll}140 & 160 & -272.65182 & 30.33 & 170 & 60 & -272.64811 & 32.66\end{array}$

$\begin{array}{llllllll}140 & 170 & -272.65153 & 30.52 & 170 & 70 & -272.64998 & 31.49\end{array}$

$\begin{array}{llllllll}140 & 180 & -272.65121 & 30.71 & 170 & 80 & -272.65081 & 30.97\end{array}$

$\begin{array}{llllllll}140 & 190 & -272.65086 & 30.93 & 170 & 90 & -272.65101 & 30.84\end{array}$

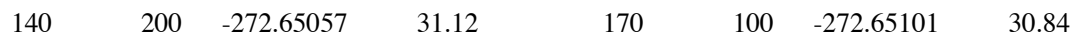

$\begin{array}{llllllll}140 & 210 & -272.65042 & 31.21 & 170 & 110 & -272.65098 & 30.86\end{array}$

$\begin{array}{llllllll}140 & 220 & -272.65040 & 31.22 & 170 & 120 & -272.65095 & 30.88\end{array}$

$\begin{array}{llllllll}140 & 230 & -272.65051 & 31.16 & 170 & 130 & -272.65090 & 30.91\end{array}$

$\begin{array}{llllllll}140 & 240 & -272.65066 & 31.06 & 170 & 140 & -272.65080 & 30.97\end{array}$

$\begin{array}{llllllll}140 & 250 & -272.65078 & 30.98 & 170 & 150 & -272.65064 & 31.07\end{array}$

$\begin{array}{llllllll}140 & 260 & -272.65084 & 30.95 & 170 & 160 & -272.65041 & 31.22\end{array}$

$\begin{array}{llllllll}140 & 270 & -272.65079 & 30.98 & 170 & 170 & -272.65012 & 31.40\end{array}$

$\begin{array}{llllllll}140 & 280 & -272.65055 & 31.13 & 170 & 180 & -272.64987 & 31.56\end{array}$

$\begin{array}{llllllll}140 & 290 & -272.65015 & 31.38 & 170 & 190 & -272.64975 & 31.63\end{array}$

$\begin{array}{llllllll}140 & 300 & -272.64961 & 31.72 & 170 & 200 & -272.64984 & 31.58\end{array}$

$\begin{array}{llllllll}140 & 310 & -272.64902 & 32.09 & 170 & 210 & -272.65006 & 31.43\end{array}$

$\begin{array}{llllllll}150 & 50 & -272.64129 & 36.94 & 170 & 220 & -272.65026 & 31.31\end{array}$

$\begin{array}{llllllll}150 & 60 & -272.64514 & 34.52 & 170 & 230 & -272.65041 & 31.22\end{array}$

$\begin{array}{llllllll}150 & 70 & -272.64840 & 32.48 & 170 & 240 & -272.65049 & 31.17\end{array}$

$\begin{array}{llllllll}150 & 80 & -272.65053 & 31.14 & 170 & 250 & -272.65050 & 31.16\end{array}$

$\begin{array}{llllllll}150 & 90 & -272.65161 & 30.46 & 170 & 260 & -272.65044 & 31.19\end{array}$

$\begin{array}{llllllll}150 & 100 & -272.65197 & 30.24 & 170 & 270 & -272.65039 & 31.23\end{array}$

$\begin{array}{llllllll}150 & 110 & -272.65201 & 30.21 & 170 & 280 & -272.65029 & 31.29\end{array}$

$\begin{array}{llllllll}150 & 120 & -272.65193 & 30.26 & 170 & 290 & -272.65010 & 31.41\end{array}$

$\begin{array}{llllllll}150 & 130 & -272.65181 & 30.34 & 170 & 300 & -272.64952 & 31.77\end{array}$

$\begin{array}{llllllll}150 & 140 & -272.65166 & 30.43 & 170 & 310 & -272.64814 & 32.64\end{array}$

$\begin{array}{llllllll}150 & 150 & -272.65149 & 30.54 & 180 & 50 & -272.64690 & 33.42\end{array}$

$\begin{array}{llllllll}150 & 160 & -272.65126 & 30.68 & 180 & 60 & -272.64907 & 32.05\end{array}$

$\begin{array}{llllllll}150 & 170 & -272.65098 & 30.86 & 180 & 70 & -272.65018 & 31.36\end{array}$

$\begin{array}{llllllll}150 & 180 & -272.65067 & 31.05 & 180 & 80 & -272.65056 & 31.12\end{array}$

$\begin{array}{llllllll}150 & 190 & -272.65033 & 31.27 & 180 & 90 & -272.65063 & 31.08\end{array}$

$\begin{array}{llllllll}150 & 200 & -272.65010 & 31.41 & 180 & 100 & -272.65065 & 31.07\end{array}$

$\begin{array}{llllllll}150 & 210 & -272.65005 & 31.44 & 180 & 110 & -272.65066 & 31.06\end{array}$

$\begin{array}{llllllll}150 & 220 & -272.65016 & 31.37 & 180 & 120 & -272.65065 & 31.07\end{array}$

$\begin{array}{llllllll}150 & 230 & -272.65033 & 31.26 & 180 & 130 & -272.65061 & 31.09\end{array}$

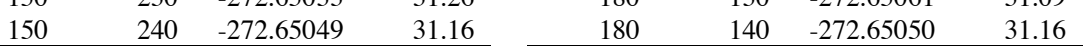

